
The Dissertation Committee for Travis Scott Metcalfe

Certifies that this is the approved version of the following dissertation:

\section{COMPUTATIONAL ASTEROSEISMOLOGY}

COMMITTEE:

R. Edward Nather, Co-Supervisor

Donald E. Winget, Co-Supervisor

Paul Charbonneau

Kepler Oliveira

J. Craig Wheeler 


\title{
COMPUTATIONAL ASTEROSEISMOLOGY
}

by

TRAVIS SCOTT METCALFE, B.S., M.A.

\author{
DISSERTATION \\ Presented to the Faculty of the Graduate School of \\ The University of Texas at Austin \\ in Partial Fulfillment \\ of the Requirements \\ for the Degree of \\ DOCTOR OF PHILOSOPHY
}

THE UNIVERSITY OF TEXAS AT AUSTIN

August 2001 
We are the stars which sing, we sing with our light;

we are the birds of fire, we fly over the sky.

- Dead Can Dance

Song of the Stars 


\section{Acknowledgements}

When I was looking for the right graduate program in astronomy during the spring of 1996, I only visited three places. Texas was the last of the three, and my visit was only a few days before the universal deadline for making a decision on where to go. I formed my first impression of Ed Nather and Don Winget while we ate lunch and talked in the WET lab during my visit. By the end of our discussion, I knew I would come to Texas and work with them.

Thanks to the unwavering support of my advisers, I am the first of my class to finish. Only four of my ten original classmates are still working toward their Ph.D.; If not for Ed and Don, I might not have continued in the program myself. I feel a great sense of privilege for having learned to be an astronomer from them. The world needs more scientists like Ed Nather and Don Winget.

In addition to our experiences in the lab, I had the pleasure of helping Ed in the classroom during his last three years of teaching. If I ever find myself in a position to teach, I will certainly aspire to be as good as Ed. By my second year as a teaching assistant for him, Ed said that I didn't need to attend the lectures anymore, but I continued going because I was getting as much out of them as the students. Besides, it allowed me to get out of our prison-like building at least a few times a week. Some of the best conversations I've had with Ed took place during our walks over to the Welch Hall classroom.

While developing the metacomputer described in this dissertation I sought advice and help from Mark Cornell, Bill Spiesman, and Gary Hansen-who also arranged for the donation of 32 computer processors from AMD. The software development owes thanks to Mike Montgomery, Paul Bradley, Matt Wood, Steve Kawaler, Peter Diener, Peter Hoeflich, and especially Paul Charbonneau and Barry Knapp for providing me with an unreleased improved version the PIKAIA 
genetic algorithm. I thank S.O. Kepler, Craig Wheeler, Atsuko Nitta and Scott Kleinman for helpful discussions, and Maurizio Salaris for providing me with data files of white dwarf internal chemical profiles.

There are many other people who influenced me in earlier stages of my career, and my dissertation project owes a debt to all of them. I'd like to thank Terry Bressi and Andrew Tubbiolo for getting me started with Linux in 1994. Brad Castalia was the first to suggest PVM to me for parallel computing, and Joe Lazio introduced me to genetic algorithms. I thank Ray White, Rob Jedicke, Dave Latham, Jim Cordes, Don McCarthy, Andrea Ghez, Todd Henry, Ted Bowen and Patrick McGuire for their part in helping me to become a good scientist.

My interest in astronomy was inspired by Darla Casey and Bonnie Osbourne, and was nurtured by Brian Montgomery, Rick Letherer, and Ed Fitzpatrick. I also owe thanks to my parents and the rest of my family in Oregon, who always believed in me and never questioned my sometimes impractical decisions. Finally, I'd like to thank Cherie Goff for helping me to maintain my sanity when times were tough, and for being the ideal companion the rest of the time.

I am grateful to the High Altitude Observatory Visiting Scientist Program for fostering this project in a very productive environment for two months during the summer of 2000. This work was supported by grant NAG5-9321 from the Applied Information Systems Research Program of the National Aeronautics \& Space Administration, and in part by grants AST 98-76730 and AST 93-15461 from the National Science Foundation.

June 2001 


\title{
COMPUTATIONAL ASTEROSEISMOLOGY
}

\author{
Publication No. \\ Travis Scott Metcalfe, Ph.D. \\ The University of Texas at Austin, 2001 \\ Co-Supervisors: R. Edward Nather and Donald E. Winget
}

White dwarf asteroseismology offers the opportunity to probe the structure and composition of stellar objects governed by relatively simple physical principles. The observational requirements of asteroseismology have been addressed by the development of the Whole Earth Telescope, but the analysis procedures still need to be refined before this technique can yield the complete physical insight that the data can provide. We have applied an optimization method utilizing a genetic algorithm to the problem of fitting white dwarf pulsation models to the observed frequencies of the most thoroughly characterized helium-atmosphere pulsator, GD 358. The free parameters in this initial study included the stellar mass, the effective temperature, the surface helium layer mass, the core composition, and the internal chemical profile.

For many years, astronomers have promised that the study of pulsating white dwarfs would ultimately lead to useful information about the physics of matter under extreme conditions of temperature and pressure. The optimization approach developed in this dissertation has allowed us to finally make good on that promise by exploiting the sensitivity of our models to the core composition. We empirically determine that the central oxygen abundance in GD 358 is $84 \pm 3$ percent. We use this value to place a preliminary constraint on the ${ }^{12} \mathrm{C}(\alpha, \gamma){ }^{16} \mathrm{O}$ nuclear reaction cross-section of $S_{300}=295 \pm 15 \mathrm{keV}$ barns.

We find a thick helium-layer solution for GD 358 that provides a better match to the data than previous fits, and helps to resolve a problem with the evolutionary connection between PG 1159 stars and DBVs. We show that the pulsation 
modes of our best-fit model probe down to the inner few percent of the stellar mass. We demonstrate the feasibility of reconstructing the internal chemical profiles of white dwarfs from asteroseismological data, and we find an oxygen profile for GD 358 that is qualitatively similar to recent theoretical calculations. This method promises to serve as a powerful diagnostic that will eventually allow us to test theories of convective overshooting and stellar crystallization. 


\section{Contents}

Acknowledgements $\quad$ iv

$\begin{array}{lll}\text { Abstract } & \text { vi }\end{array}$

List of Tables $\quad$ x

List of Figures $\quad$ xi

Chapter 1 Context 1

1.1 Introduction . . . . . . . . . . . . . . . 1

1.2 What Good is Astronomy? . . . . . . . . . . . . . 2

1.3 The Nature of Knowledge . . . . . . . . . . . . 3

1.4 The Essence of my Dissertation Project . . . . . . . . . . . . 4

1.4.1 Genetic Algorithms . . . . . . . . . . . 5

1.4.2 White Dwarf Stars ............. 5

1.4.3 Linux Metacomputer ............. 8

1.4.4 The Big Picture . . . . . . . . . . . . . . . 10

1.5 Organization of this Dissertation . . . . . . . . . 10

$\begin{array}{llr}\text { Chapter } 2 & \text { Linux Metacomputer } & 12\end{array}$

2.1 Introduction . . . . . . . . . . . . . . 12

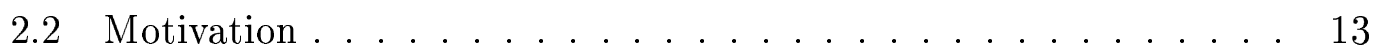

2.3 Hardware . . . . . . . . . . . . . . . 13

2.3.1 Master Computer . . . . . . . . . . . 15

2.3.2 Slave Nodes . . . . . . . . . . . . . 16

2.4 Software ............................. 17 
$2.4 .1 \quad \operatorname{Linux} \ldots \ldots \ldots \ldots \ldots \ldots \ldots \ldots$

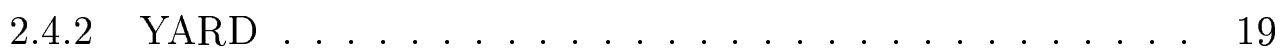

$2.4 .3 \quad$ NETBOOT . . . . . . . . . . . . . . . . . . 19

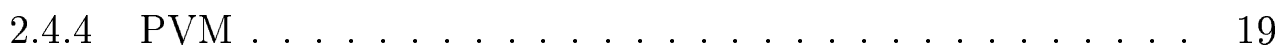

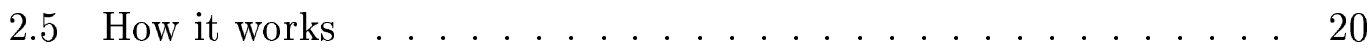

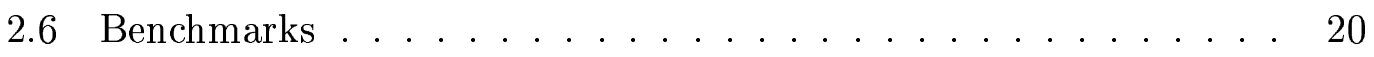

2.7 Stumbling Blocks $\ldots \ldots \ldots \ldots \ldots \ldots \ldots \ldots$

Chapter 3 Parallel Genetic Algorithm 22

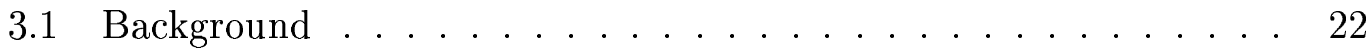

3.2 Genetic Algorithms . . . . . . . . . . . . . . . . . 23

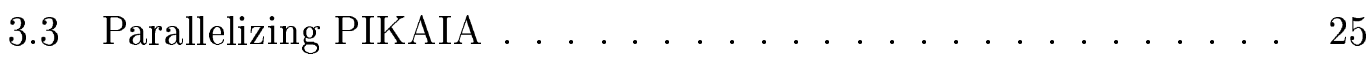

$3.3 .1 \quad$ Parallel Virtual Machine . . . . . . . . . . . . . . 26

3.3 .2 The PIKAIA Subroutine . . . . . . . . . . . . . 27

3.4 Master Program . . . . . . . . . . . . . . . . . . . . . 28

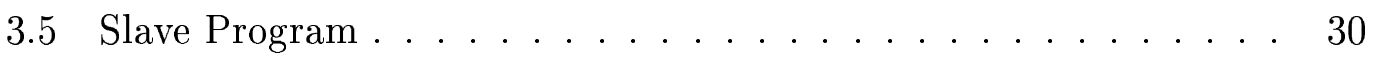

Chapter 4 Forward Modeling 32

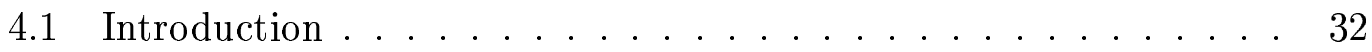

4.2 The DBV White Dwarf GD $358 \ldots \ldots \ldots \ldots$

4.3 DBV White Dwarf Models . . . . . . . . . . . . 33

4.3.1 Defining the Parameter-Space . . . . . . . . . . . 33

4.3.2 Theoretical Models . . . . . . . . . . . . . . . 35

$4.4 \quad$ Model Fitting . . . . . . . . . . . . . . . . 37

4.4.1 Application to Noiseless Simulated Data . . . . . . . . 37

4.4 .2 The Effect of Gaussian Noise . . . . . . . . . . . . 39

4.4 .3 Application to GD $358 \ldots \ldots \ldots$. . . . . . . . 41

4.5 Initial Results . . . . . . . . . . . . . . . . . . 42

4.6 Internal Composition \& Structure . . . . . . . . . . . 46

4.7 Constraints on Nuclear Physics _. . . . . . . . . . . . . 49

$\begin{array}{lll}\text { Chapter } 5 & \text { Reverse Approach } & 51\end{array}$

5.1 Introduction . . . . . . . . . . . . . . . 51

5.2 Model Perturbations . . . . . . . . . . . . . . 53 
5.2 .1 Proof of Concept . . . . . . . . . . . . . . 53

5.2 .2 Application to GD $358 \ldots \ldots \ldots \ldots \ldots$

5.3 Results . . . . . . . . . . . . . . . . 56

5.4 Chemical Profiles . . . . . . . . . . . . . . 58

Chapter 6 Conclusions $\quad 60$

6.1 Discussion of Results . . . . . . . . . . . . . 6 60

6.2 The Future . . . . . . . . . . . . . . . . 64

6.2.1 Next Generation Metacomputers . . . . . . . . . . 64

6.2 .2 Code Adaptations . . . . . . . . . . . . . . . 65

6.2 .3 More Forward Modeling . . . . . . . . . . . . 66

6.2.4 Ultimate Limits of Asteroseismology _. . . . . . . . 67

6.3 Overview . . . . . . . . . . . . . . . . . 67

$\begin{array}{ll}\text { Appendix A Observations for the WET } & 69\end{array}$

A.1 What is the WET? . . . . . . . . . . . . . 70

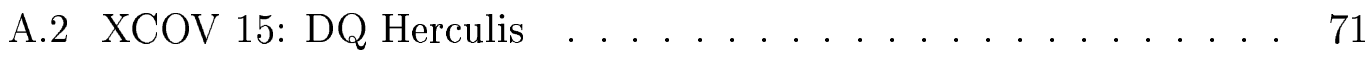

A.3 XCOV 17: BPM $37093 \ldots \ldots \ldots \ldots \ldots \ldots \ldots$

A.4 XCOV 18: HL Tau $76 \ldots \ldots \ldots \ldots \ldots \ldots \ldots$

A.5 XCOV 19: GD $358 \ldots \ldots \ldots \ldots \ldots \ldots \ldots \ldots$

$\begin{array}{ll}\text { Appendix B Interactive Simulations } & \mathbf{7 2}\end{array}$

B.1 Pulsation Visualizations . . . . . . . . . . . . . . . . 73

B.2 The Effect of Viewing Angle . . . . . . . . . . . 73

Appendix C Computer Codes $\quad \mathbf{7 4}$

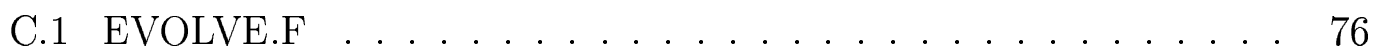

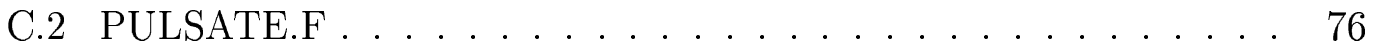

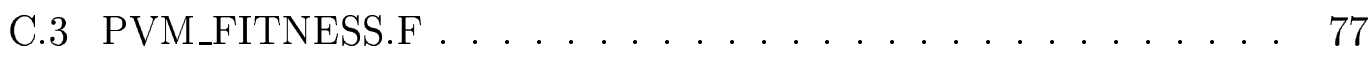

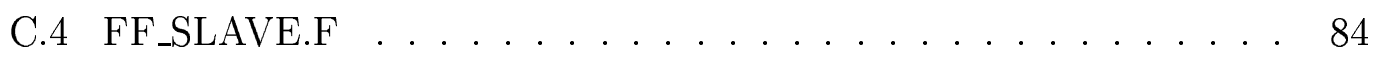

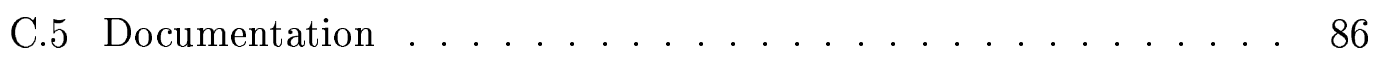

$\begin{array}{ll}\text { Bibliography } & 87\end{array}$

$\begin{array}{ll}\text { Vita } & 92\end{array}$ 


\section{List of Tables}

2.1 The Absolute Speed of the Metacomputer . . . . . . . . . 21

4.1 Results for Noiseless Simulated Data . . . . . . . . . . 38

4.2 Results for GD 358 Data . . . . . . . . . . . . . . 45

4.3 Convergence of the Method on Simulated Data. . . . . . . . . 48

5.1 Periods and Period Spacings for GD 358 and Best-fit Models . . 56 


\section{List of Figures}

1.1 Hubble Space Telescope image of a hot white dwarf . . . . . . 7

1.2 Sample light curve of the pulsating white dwarf GD $358 \ldots$. . . 8

1.3 The parallel computer built and used for this thesis . . . . . . 9

2.1 The master computer surrounded by 64 slave nodes . . . . . . . 14

2.2 Darwin, the master computer. . . . . . . . . . . 15

2.3 A view inside one of the metacomputer nodes . . . . . . . . 16

3.1 Flow chart for the parallel fitness evaluation subroutine . . . . 29

3.2 Flow chart for the slave program . . . . . . . . . . 30

4.1 Linear cut imposed on the helium layer mass . . . . . . . . . 35

4.2 Two determinations of the noise on pulsation frequencies . . . . . 40

4.3 General features of the 3-dimensional parameter-space . . . . . . 43

4.4 Parameter-space for GD 358 using various core compositions . . . 44

4.5 Uncertainty on the central oxygen mass fraction in GD 358 . . . 49

5.1 Sensitivity of pulsation periods to the deep internal structure . . . 52

5.2 The Brunt-Väisälä frequency for various chemical profiles . . . . . 54

5.3 GD 358 pulsation periods with various best-fit models . . . . . . 57

5.4 Internal chemical profiles of the best-fit models to GD 358 . . . 59

A.1 The Whole Earth Telescope . . . . . . . . . . 70 


\section{Chapter 1}

\section{Context}

"In learning any subject of a technical nature where mathematics plays a role...it is easy to confuse the proof itself with the relationship it establishes. Clearly, the important thing to learn and to remember is the relationship, not the proof."

-Richard Feynman

\section{$1.1 \quad$ Introduction}

There isn't much point in writing a dissertation if only a few people in the world understand it, much less care enough about the subject to read every word. It takes a long time to be educated as an astronomer, and by the time it's over most students have internalized the basic concepts. It's easy to forget the mental hurdles that challenged us along the way.

I began formal study in astronomy about ten years ago, and at every step along the way my education has been subsidized by taxpayers. It seems only fair that I should try to give something in return. I've decided to use the first chapter of my dissertation to place my research project into a larger social context. I will do my best to ensure that this chapter is comprehensible to the people who so graciously and unknowingly helped me along the way. It's the least I can do, and maybe it will convince some of them that their investment was worthwhile. 


\subsection{What Good is Astronomy?}

How can astronomers justify the support they receive from taxpayers? What benefit does society derive from astronomical research? What is the rate of return on their investment? These questions are difficult, but not impossible, to answer. The economic benefits of basic scientific research are often realized over the long-term, and it's hard to anticipate what spin-offs may develop as a result of any specific research program.

A purist might refuse even to respond to these questions. What justification does basic research need other than the pursuit of knowledge? What higher achievement can civilization hope to accomplish than the luxury of seeking answers to some of the oldest questions: where did we come from, and what is our place in the universe?

The proper response is probably somewhere in between. Over the years, advances in scientific knowledge made possible through basic research have had a definite impact on the average citizen, but the magnitude of this impact is difficult to predict at the time the research is proposed. As a result, much of the basic research funded by the public often sounds ridiculous to many taxpayers. Gradually, this has led to a growing reluctance by the public to fund basic research, and the annual budgets of government funding agencies have stagnated as a consequence. Public education is an essential component of any strategy to treat this problem effectively.

I contend that the money taxpayers contribute to scientific research in some sense obligates the researchers to make their work accessible to the public. Some combination of teaching and public outreach by researchers should provide an adequate return on the investment. If this doesn't seem reasonable, put it in perspective by looking at exactly how much it costs U.S. taxpayers to fund astronomical research:

- In the year 2000 , the total federal budget amounted to $\$ 1.88$ trillion $^{1}$

- Revenue from personal income taxes amounted to $\$ 900$ billion $^{1}$

\footnotetext{
${ }^{1}$ http://w3.access.gpo.gov/usbudget/fy2000/table2_1.gif
} 
- The "non-defense discretionary" portion of the budget totaled $\$ 330$ billion $^{2}$

- The National Science Foundation (NSF) budget was $\$ 3.95$ billion $^{3}$

- The NSF allocation to the Directorate for Mathematics \& Physical Sciences (MPS) amounted to $\$ 754$ million $^{4}$

- The MPS allocation to Astronomical Sciences amounted to $\$ 122$ million ${ }^{4}$

So, even if you assume that the revenue for "non-defense discretionary" comes entirely from personal income taxes, funding astronomy is cheap. Out of every $\$ 1000$ in revenue from personal income taxes, $\$ 365$ goes into the non-defense discretionary fund. About $\$ 4.35$ ends up in the hands of the National Science Foundation. Of this, 83 cents goes to fund all of Mathematics \& Physical Sciences. In the end, for every $\$ 1000$ in taxes only 13 cents ends up funding Astronomical Sciences.

\subsection{The Nature of Knowledge}

Science is chiefly concerned with accumulating knowledge. What does this mean? The Greek philosopher Plato defined knowledge as "justified true belief". Belief by itself is what we commonly call faith. There's nothing wrong with faith, but it doesn't constitute knowledge under Plato's definition. A belief that is justified but false is simply a misconception. Based on incomplete information I may be justified in believing that the Earth is flat, but I cannot know this to be so because it turns out not to be true. Likewise, I may believe something that turns out to be true even though I had no justification for believing it. For example, I cannot know that a fair coin toss will turn up heads even if it does in fact turn up heads, because I can have no defensible justification for this belief.

In science, our justification for believing something is usually based on observations of the world around us. The observations can occur either before or

\footnotetext{
${ }^{2}$ http://w3.access.gpo.gov/usbudget/fy2000/table2_2.gif

${ }^{3}$ http://www.nsf.gov/bfa/bud/fy2000/overview.htm

${ }^{4}$ http://www.nsf.gov/bfa/bud/fy2000/DirFund/mps.htm
} 
after we have formulated a belief, corresponding to two broad methods of reasoning. In deductive reasoning, we begin by formulating a theory and deriving specific hypothetical consequences that can be tested. We gather observations to test the hypotheses and help to either confirm or refute the theory. In most cases the match between observations and theory is imperfect, and we refine the theory to try to account for the differences. Einstein's theory of relativity is a good example of this type of reasoning. Based on some reasonable fundamental assumptions, Einstein developed a theory of the geometry of the universe. He predicted some observational consequences of this theory and people tested these predictions experimentally.

For inductive reasoning, we begin by looking for patterns in existing observations. We come up with some tentative hypotheses to explain the patterns, and ultimately develop a general theory to explain the observed phenomena. Kepler's laws of planetary motion are good examples of inductive reasoning. Based on the precise observations of the positions of planets in the night sky made by Tycho Brahe, Kepler noticed some regular patterns. He developed several empirical laws that helped us to understand the complex motions of the planets, which ultimately inspired Newton to develop a general theory of gravity.

Armed with these methods of developing and justifying our beliefs, we slowly converge on the truth. However, it's important to realize that we may never actually arrive at our goal. We may only be able to find better approximations to the truth. In astronomy we do not have the luxury of designing the experiments or manipulating the individual components, so knowledge in the strict sense is even more difficult to obtain. Fortunately, the universe contains such a vast and diverse array of phenomena that we have plenty to keep us occupied.

\subsection{The Essence of my Dissertation Project}

When I originally conceived of my dissertation project three years ago, the title of my proposal was Genetic-Algorithm-based Optimization of White Dwarf Pulsation Models using an Intel/Linux Metacomputer. That's quite a mouthful. It's actually much less intimidating than it sounds at first. Let me explain what this project is really about, one piece at a time. 


\subsubsection{Genetic Algorithms}

Given the nature of knowledge, astronomers generally need to do two things to learn anything useful about the universe. First, we need to gather quantitative observations of something in the sky, usually with a telescope and some sophisticated electronic detection equipment. Second, we need to interpret the observations by trying to match them with a mathematical model, using a computer. The computer models have many different parameters - sort of like knobs and switches that can be adjusted - and each represents some aspect of the physical laws that govern the behavior of the model.

When we find a model that seems to match the observations fairly well, we assume that the values of the parameters tell us something about the true nature of the object we observed. The problem is: how do we know that some other combination of parameters won't do just as well, or even better, than the combination we found? Or what if the model is simply inadequate to describe the true nature of the object?

The process of adjusting the parameters to find a "best-fit" model to the observations is essentially an optimization problem. There are many well established mathematical tools (algorithms) for doing this - each with strengths and weaknesses. I am using a relatively new approach that uses a process analogous to Charles Darwin's idea of evolution through natural selection. This so-called genetic algorithm explores the many possible combinations of parameters, and finds the best combination based on objective criteria.

\subsubsection{White Dwarf Stars}

What is a white dwarf star? To astronomers, dwarf is a general term for smaller stars. The color of a star is an indication of the temperature at its surface. Very hot objects emit more blue-white light, while cooler objects emit more red light. Our Sun is termed a yellow dwarf and there are many stars cooler than the Sun called red dwarfs. So a white dwarf is a relatively small star with a very hot surface.

In 1844, an astronomer named Friedrich Bessel noticed that Sirius, the brightest star in the sky, appeared to wobble slightly as it moved through space. He 
inferred that there must be something in orbit around it. Sure enough, in 1862 the faint companion was observed visually by Alvan Clark (a telescope maker) and was given the name "Sirius B". By the 1920's, the companion had completed one full orbit of Sirius and its mass was calculated, using Newton's laws, to be roughly the same as the Sun. When astronomers measured its spectrum, they found that it emitted much more blue light than red, implying that it was very hot on the surface even though it didn't appear very bright in the sky. These observations implied that it had to be a million times smaller than a regular star with the same mass as the Sun-the first white dwarf!

The exact process of a star becoming a white dwarf depends on the mass of the star, but all stars less massive than about 8 times the mass of the Sun (99\% of all stars) will eventually become white dwarfs. Normal stars fuse hydrogen into helium until the hydrogen deep in the center begins to run out. For very massive stars this may take only 1 million years - but for stars like the Sun the hydrogen lasts for 10,000 million years. When enough helium collects in the middle of the star, it becomes a significant source of extra heat. This messes up the internal balance of the star, which then begins to bloat into a so-called red giant.

If the star is massive enough, it may eventually get hot enough in the center to fuse the helium into carbon and oxygen. The star then enjoys another relatively stable period, though much shorter this time. The carbon and oxygen, in their turn, collect in the middle. If the star isn't massive enough to reach the temperature needed to fuse carbon and oxygen into heavier elements, then these elements will simply continue to collect in the center until the helium fuel runs out. In the end, you have a carbon/oxygen white dwarf surrounded by the remains of the original star (see Figure 1.1).

In normal stars like the Sun, the inward pull of gravity is balanced by the outward push of the high-temperature material in the center, fusing hydrogen into helium and releasing energy in the process. There is no nuclear fusion in a white dwarf. Instead, the force that opposes gravity is called "electron degeneracy pressure".

When electrons are squeezed very close together, the energy-states that they would normally be able to occupy become indistinguishable from the energystates of neighboring electrons. The rules of quantum mechanics tell us that 


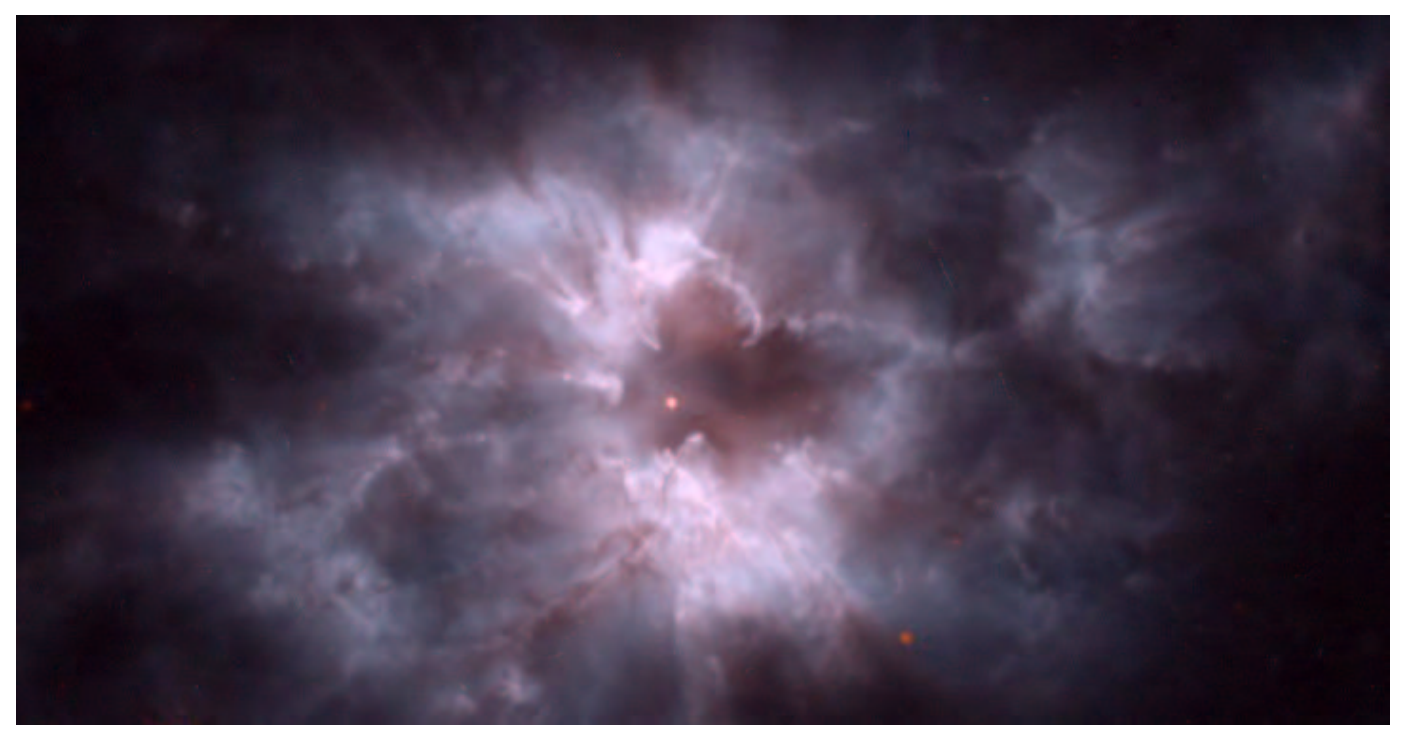

Figure 1.1: A hot white dwarf at the center of the planetary nebula NGC 2440. This image was obtained with the Hubble Space Telescope by H. Bond (STScI) and R. Ciardullo (PSU).

no two electrons can occupy exactly the same energy-state, and as the average distance between electrons gets smaller the average momentum must get larger. So, the electrons are forced into higher energy-states (pushed to higher speeds) just because of the density of the matter.

This quantum pressure can oppose gravity as long as the density doesn't get too high. If a white dwarf has more than about 1.4 times the mass of the Sun squeezing the material, there will be too few energy-states available to the electrons (since they cannot travel faster than the speed of light) and the star will collapse - causing a supernova explosion.

\section{Pulsating White Dwarfs}

Some white dwarfs show very regular variations in the amount of light reaching our telescopes (see Figure 1.2). The pattern of this variation suggests that these white dwarfs are pulsating - as if there are continuous star-quakes going on. By studying the patterns of light variation, astronomers can learn about the interior structure of white dwarfs - in much the same way as seismologists can learn about the inside of the Earth by studying earthquakes. For this reason, the study of 


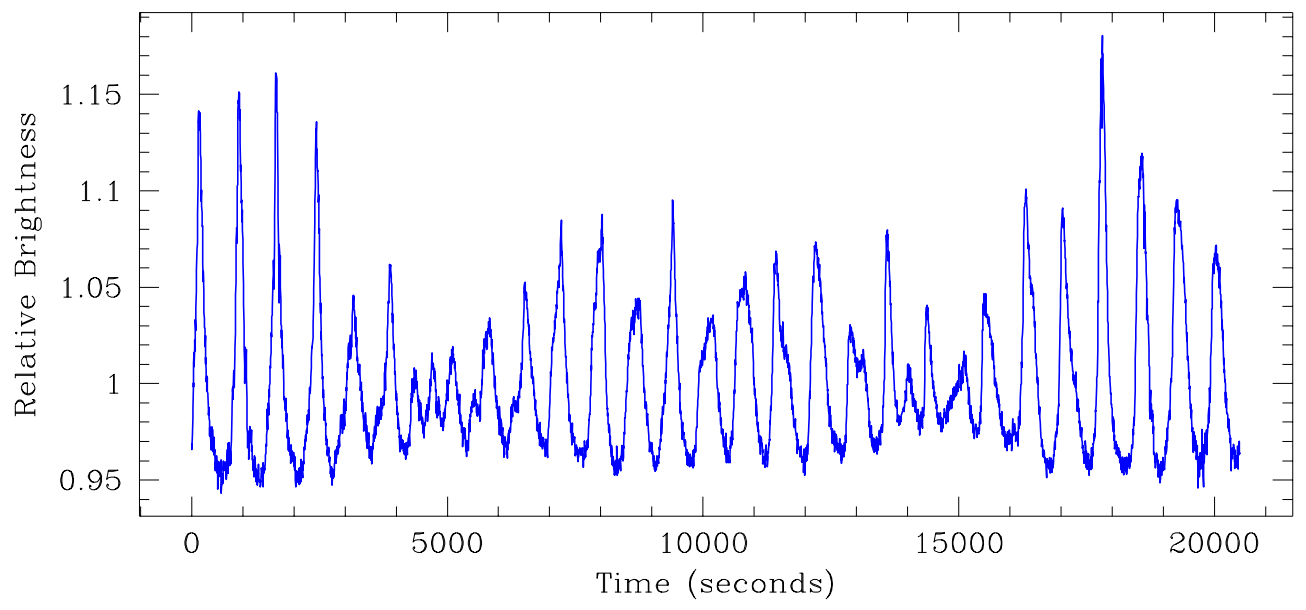

Figure 1.2: Non-radial pulsations in some white dwarf stars cause periodic changes in their brightness over time. This sample light curve of GD 358 shows many pulsation periods excited simultaneously, causing beating and a total variation of about 10 percent on timescales shorter than an hour.

these pulsating white dwarfs is called asteroseismology.

Since 1988, very useful observations of pulsating white dwarfs have been obtained with the Whole Earth Telescope - a collaboration of astronomers around the globe who cooperate to monitor these stars for weeks at a time. I have helped to make some of these observations, but I have also worked on interpreting them using our computer models. I have approached the models in two ways:

- I assume the models are accurate representations of the real white dwarf stars, and I try to find the combination of model parameters that do the best job of matching the observations.

- I assume the models are incomplete representations of the real white dwarf stars, and I try to find changes to the internal structure of the models that yield an improved match to the observations.

\subsubsection{Linux Metacomputer}

The dictionary definition of the prefix meta- is: "Beyond; More comprehensive; More highly developed." So a meta-computer goes beyond the boundaries of a 
traditional computer as we are accustomed to thinking of it. Essentially, a metacomputer is a collection of many individual computers, connected by a network (the Internet for example), which can cooperate on solving a problem. In general, this allows the problem to be solved much more quickly than would be possible using a single computer.

Supercomputers are much faster than a single desktop computer too, but they usually cost millions of dollars, and everyone has to compete for time to work on their problem. Recently, personal computers have become very fast and relatively inexpensive. At the same time, the idea of free software (like the Linux operating system) has started to catch on. These developments have made it feasible to build a specialized metacomputer with as much computing power as a 5 -year-old supercomputer, but for only about $1 \%$ of the cost!

The problem that I am working on has required that I run literally millions of computer models of pulsating white dwarf stars over the several-year duration of my research project. To make these calculations practical, I configured a metacomputer using 64 minimal PC systems running under a customized version of the Linux operating system (see Figure 1.3).

Thanks to another piece of free software called PVM (for Parallel Virtual Machine), I can use one fully-equipped personal computer to control the entire

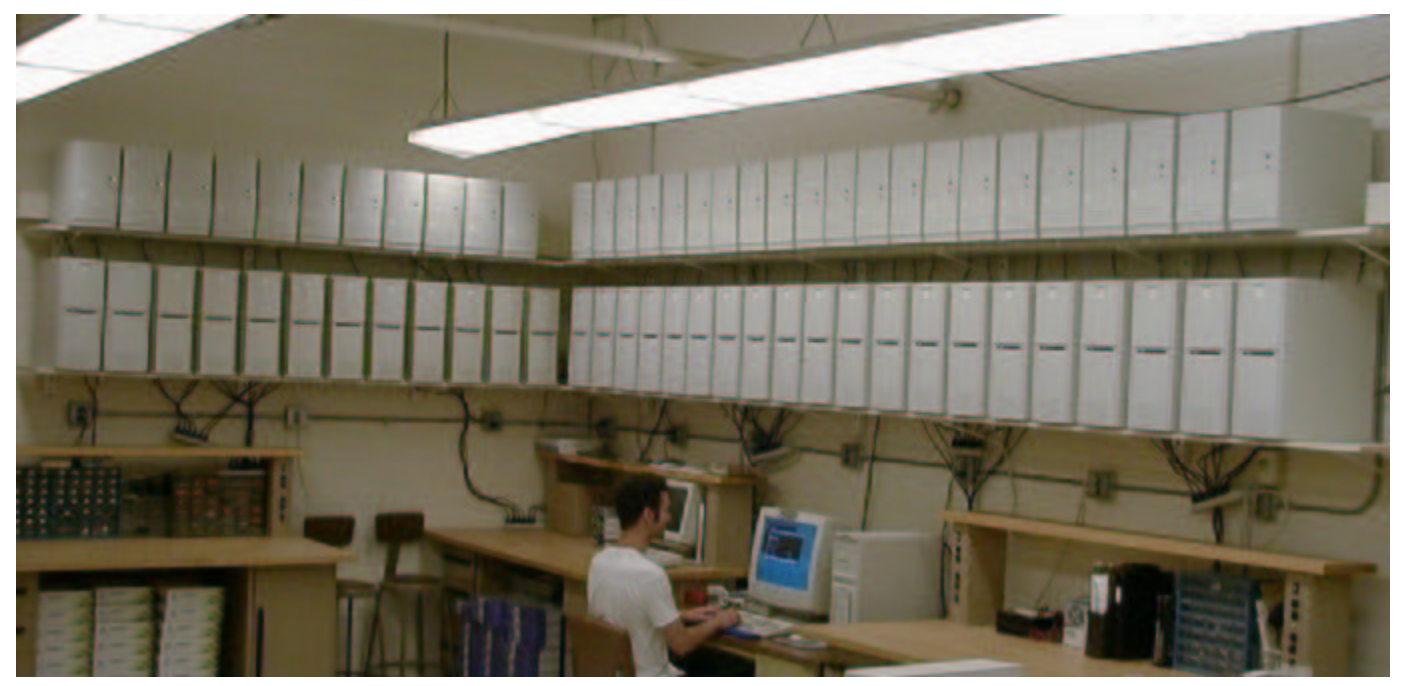

Figure 1.3: This metacomputer is a collection of 64 minimal PCs connected by a network, and can calculate our white dwarf models in parallel. 
system. This central computer is responsible for distributing work to each of the 64 processors, and collecting the results. There is a small amount of work required just to keep track of everything, so the metacomputer actually runs about 60 (rather than 64) times as fast as a single system. Not bad!

\subsubsection{The Big Picture}

So I'm using a relatively new optimization method to find the best set of parameters to match the observations with our computer models of pulsating white dwarf stars; and there are so many models to run that I need a lot of computing power, so I linked a bunch of PC systems together to do the job. But what do I hope to learn?

Well, the source of energy for regular stars like the Sun is nuclear fusion. This is the kind of nuclear energy that doesn't produce any dangerous radioactive waste. Astronomers have a good idea of how fusion energy works to power stars, but the process requires extremely high temperatures and pressures, which must be sustained for a long time; these conditions are difficult to reproduce (in a controlled way) in laboratories on Earth. Physicists have been working on it for several decades, but sustained nuclear fusion has still never been achieved. This leads us to believe that we may not understand all of the physics that we need to make fusion work. If scientists could learn to achieve controlled nuclear fusion, it would provide an essentially inexhaustible source of clean, sustainable energy.

To help ensure that we properly understand how stars work, it is useful to look at the "ashes" of the nuclear fusion. Those ashes are locked in the white dwarf stars, and asteroseismology allows us to peer down inside and probe around. But our understanding can only be as good as our models, so it is important both to make sure that we find the absolute "best" match, and to figure out what limitations are imposed simply by using the models we use. It's only one piece of the puzzle, but it's a place to start.

\subsection{Organization of this Dissertation}

Most of the work presented in this dissertation has already been published. Each chapter should be able to stand by itself, and together they tell the story of how 
I've spent the last three years of my professional life.

Chapter 2 describes the development of the Linux metacomputer and provides a detailed account of its inner workings. The text is derived from articles published in the Linux Journal, Baltic Astronomy, and a Metacomputer mini-ноwто posted on the world-wide web.

Chapter 3 includes a more detailed background on genetic algorithms. I outline the steps I took to create a parallel version of a general-purpose genetic algorithm in the public domain, and its implementation on the Linux metacomputer.

Chapter 4 is derived primarily from a paper published in the Astrophysical Journal in December 2000. It describes the first application of the genetic algorithm approach to model pulsations in the white dwarf GD 358, and an extension of the method to determine its internal composition and structure.

Chapter 5 comes from a paper published in the Astrophysical Journal in August 2001. It describes a method of "reverse engineering" the internal structure of a pulsating white dwarf by using the genetic algorithm and the models in a slightly different way.

Chapter 6 sums up the major conclusions of this work and outlines future directions. The appendices contain an archive of my observations for the Whole Earth Telescope, some interactive simulations of pulsating white dwarfs, and an archive of the computer codes used for this dissertation. 


\section{Chapter 2}

\section{Linux Metacomputer}

"There's certainly a strong case for people disliking Microsoft because their operating systems... suck."

-Linus Torvalds

\section{$2.1 \quad$ Introduction}

The adjustable parameters in our computer models of white dwarfs presently include the total mass, the temperature, hydrogen and helium layer masses, core composition, convective efficiency, and internal chemical profiles. Finding a proper set of these to provide a close fit to the observed data is difficult. The traditional procedure is a guess-and-check process guided by intuition and experience, and is far more subjective than we would like. Objective procedures for determining the best-fit model are essential if asteroseismology is to become a widely-accepted and reliable astronomical technique. We must be able to demonstrate that for a given model, within the range of different values the model parameters can assume, we have found the only solution, or the best one if more than one is possible. To address this problem, we have applied a searchand-fit technique employing a genetic algorithm (GA), which can explore the myriad parameter combinations possible and select for us the best one, or ones (cf. Goldberg, 1989; Charbonneau, 1995; Metcalfe, 1999). 


\subsection{Motivation}

Although genetic algorithms are often more efficient than other global techniques, they are still quite demanding computationally. On a reasonably fast computer, it takes about a minute to calculate the pulsation periods of a single white dwarf model. However, finding the best-fit with the GA method requires the evaluation of hundreds of thousands of such models. On a single computer, it would take more than two months to find an answer. To develop this method on a reasonable timescale, we realized that we would need our own parallel computer.

It was January 1998, and the idea of parallel computing using inexpensive personal computer (PC) hardware and the free Linux operating system started getting a lot of attention. The basic idea was to connect a bunch of PCs together on a network, and then to split up the computing workload and use the machines collectively to solve the problem more quickly. Such a machine is known to computer scientists as a metacomputer. This differs from a supercomputer, which is much more expensive since all of the computing power is integrated into a single unified piece of hardware.

There are several advantages to using a metacomputer rather than a more traditional supercomputer. The primary advantage is price: a metacomputer that is just as fast as a 5-year-old supercomputer can be built for only about 1 percent of the cost - about $\$ 10,000$ rather than $\$ 1$ million! Another major advantage is access: the owner and administrator of a parallel computer doesn't need to compete with other researchers for time or resources, and the hardware and software configuration can be optimized for a specific problem. Finally if something breaks, replacement parts are standard off-the-shelf components that are widely available, and while they are on order the computer is still functional at a slightly reduced capacity.

\subsection{Hardware}

The first Linux metacomputer, known as the Beowulf cluster ${ }^{5}$ (Becker et al., 1995), has now become the prototype for many general-purpose Linux clusters.

\footnotetext{
${ }^{5}$ http://www.beowulf.org/
} 
Our machine is similar to Beowulf in the sense that it consists of many independent PCs, or nodes; but our goal was to design a special-purpose computational tool with the best performance possible per dollar, so our machine differs from Beowulf in several important ways.

We wanted to use each node of the metacomputer to run identical tasks (white dwarf pulsation models) with small, independent sets of data (the parameters for each model). The results of the calculations performed by the nodes consisted of just a few numbers (the root-mean-square differences between the observed and calculated pulsation periods) which only needed to be communicated to the master process (the genetic algorithm), never to another node. Essentially, network bandwidth was not an issue because the computation to communication ratio of our application was extremely high, and hard disks were not needed on the nodes because our problem did not require any significant amount of data storage. We settled on a design including one master computer and 64 minimal nodes connected by a simple coaxial network (see Figure 2.1).

We developed the metacomputer in four phases. To demonstrate that we could make the system work, we started with the master computer and only two nodes. When the first phase was operational, we expanded it to a dozen nodes to

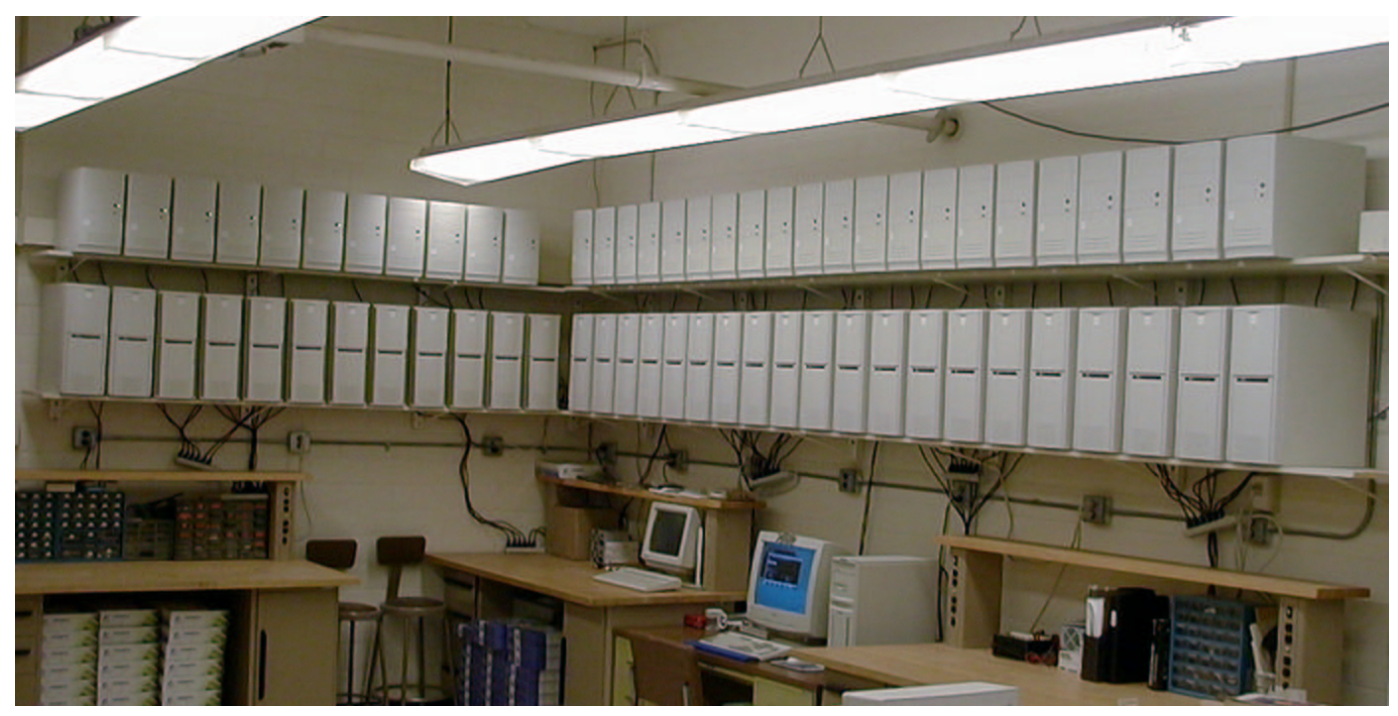

Figure 2.1: The 64 minimal nodes of the metacomputer on shelves surrounding the master computer. 
demonstrate that the performance would scale. In the third phase, we occupied the entire bottom shelf with a total of 32 nodes. Months later, we were given the opportunity to expand the system by an additional 32 nodes with processors donated by AMD and we filled the top shelf, yielding a total of 64 nodes.

\subsubsection{Master Computer}

Our master computer, which we call Darwin, is a Pentium-II $333 \mathrm{MHz}$ system with $128 \mathrm{MB}$ RAM and two $8.4 \mathrm{~GB}$ hard disks (see Figure 2.2). It has three NE2000 compatible network cards, each of which drives $1 / 3$ of the nodes on a subnet. No more than 30 devices (e.g. ethernet cards in the nodes) can be included on a single subnet without using a repeater to boost the signal. Additional ethernet cards for the master computer were significantly less expensive than a repeater.

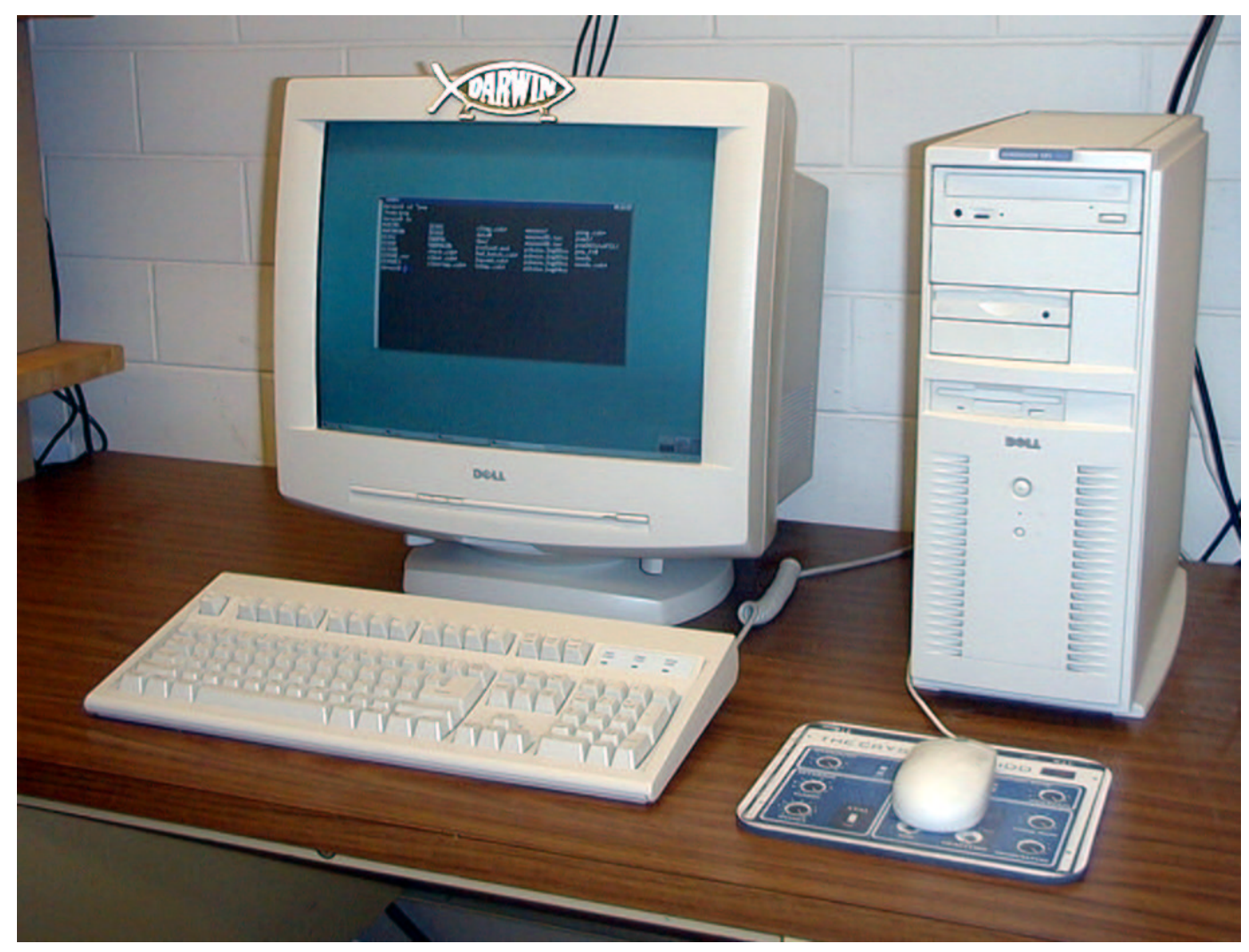

Figure 2.2: Darwin, the master computer controlling all 64 nodes. 


\subsubsection{Slave Nodes}

We assembled the nodes from components obtained at a local discount computer outlet. Each node includes only an ATX tower case and power supply with a motherboard, a processor and fan, a single 32 MB RAM chip, and an NE-2000 compatible network card (see Figure 2.3). Half of the nodes contain Pentium-II $300 \mathrm{MHz}$ processors, while the other half are AMD K6-II $450 \mathrm{MHz}$ chips. We added inexpensive Am27C256 $32 \mathrm{~kb}$ EPROMs (erasable programmable read-only memory) to the bootrom sockets of each network card. The nodes are connected in series with 3 -ft ethernet coaxial cables, and the subnets have $50 \Omega$ terminators on each end. The total cost of the system was around $\$ 25,000$ but it could be built for considerably less today, and less still tomorrow.

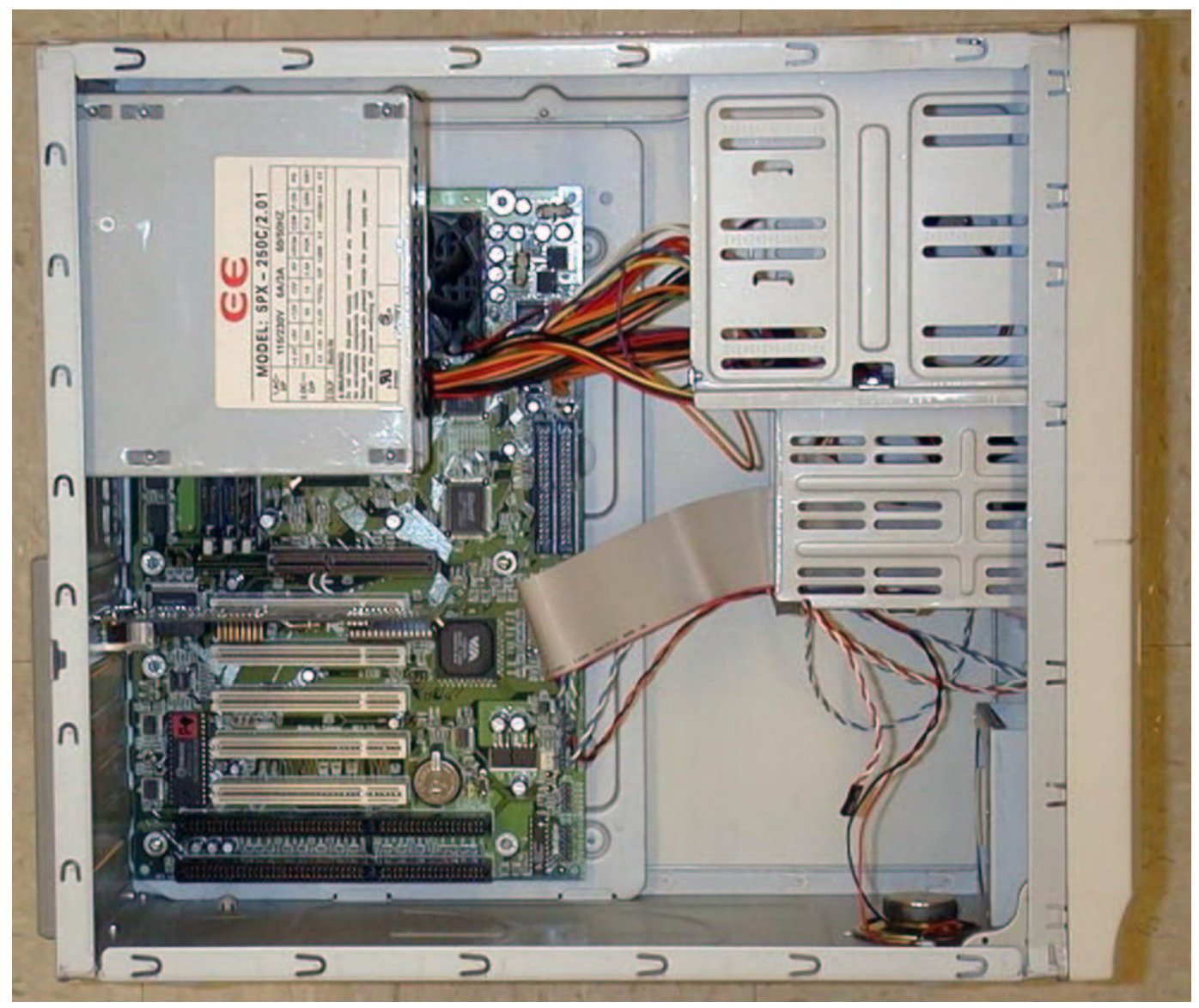

Figure 2.3: A view inside one of the metacomputer nodes. 


\subsection{Software}

Configuring the software was not much more complicated than setting up a diskless Linux system. The main difference was that we wanted to minimize network activity by giving each node an identical, independent filesystem rather than mounting a shared network filesystem. Since the nodes had no hard disks, we needed to create a self-contained filesystem that could be downloaded and mounted in a modest fraction of the 32 MB RAM.

To make the system work, we relied heavily on the open-source Linux operating system and other software packages that were available for free on the Internet. A piece of software called YARD allowed us to create the minimal Linux filesystems that we needed for each node to run independently. We used a package called NETBOOT to program the EPROM chips; this allowed each node to automatically download and boot the filesystem, which was mounted in part of the RAM. Finally, we used the PVM software to exploit the available resources and take care of the communication required to manage the parallel processing operations.

\subsubsection{Linux}

In 1991, a young student named Linus Torvalds at the University of Helsinki in Finland created a free Unix-like operating system as a hobby. He posted his work on the Internet and, together with a small group of friends, he continued to develop it. In 1994, version 1.0 of "Linux" was released. Today, millions of people worldwide use Linux as an alternative to the operating systems sold by Microsoft (Windows) and Sun Microsystems (Solaris). Unlike these more common operating systems, the source code for Linux is freely available to everyone.

The computer code used to create the Linux operating system is known as the kernel. To ensure that the hardware components of our nodes would be recognized by the operating system, we custom compiled the Linux 2.0.34 kernel. We included support for the NE-2000 ethernet card, and specified that the filesystem was on the network and should be retrieved using the bootp protocol (see below) and mounted in RAM.

Getting the master computer to recognize its three ethernet cards required 
extra options to be passed to the kernel at boot time. We specified the addresses of these devices and passed them to the Linux kernel through LOADLIN, a DOSbased program that boots up Linux.

Each card on the network needed to be assigned a unique IP (Internet Protocol) address, which is a sequence of four numbers between 0 and 255 separated by periods. The IP addresses that are reserved for subnets (which do not operate on the Internet) are:

$$
\begin{array}{ll}
\text { 10.0.0.0 } & \text { (Class A network) } \\
\text { 172.16.0.0 } \rightarrow \text { 172.31.0.0 } & \text { (Class B network) } \\
\text { 192.168.0.0 } \rightarrow \text { 192.168.255.0 } & \text { (Class C network) }
\end{array}
$$

Since we were dealing with a relatively small number of machines, we used the first three numbers to specify the domain (pvm.net), and the last number to specify the hostname (e.g. node001). Our first ethernet card (eth1) was assigned control of the 192.168.1.0 subnet while 192.168.2.0 and 192.168.3.0 were handled by eth2 and eth3 respectively.

We used the Bootstrap Protocol (bootp) and the Trivial File Transfer Proto$\mathrm{col}(\mathrm{tftp})$ to allow the nodes to retrieve and boot their kernel, and to download a compressed version of their root filesystem. We relied heavily on Robert Nemkin's Diskless HOWTO ${ }^{6}$ to make it work.

The main configuration file for bootp is /etc/bootptab, which contains a list of the hostnames and IP addresses that correspond to each ethernet card on the subnet. Each card is identified by a unique hardware address - a series of 12 hexadecimal numbers (0-9,a-f) assigned by the manufacturer. In addition to various network configuration parameters, this file also describes the location of the bootimage to retrieve with tftp. Since each node is running an identical copy of the bootimage, setting up tftp was considerably easier than it would have been in general. We simply created a /tftpboot directory on the server and placed a copy of the bootimage there.

\footnotetext{
${ }^{6} \mathrm{http}: / /$ www.linuxdoc.org/HOWTO/Diskless-HOWTO.html
} 


\subsubsection{YARD}

To create the self-contained root filesystem, we used Tom Fawcett's YARD (Yet Another Rescue Disk) package ${ }^{7}$. This piece of software was designed to make rescue disks - self-contained minimal filesystems that can fit on a single floppy disk and be used in emergencies to boot and fix problems on a Linux system. Since the white dwarf pulsation code does not require a great deal of system memory to run, we were free to use half of the 32 MB RAM for our filesystem, which allowed us to include much more than would fit on a floppy disk.

There are two files that control the properties and content of the YARD filesystem: Config.pl and Bootdisk_Contents. The Config.pl file controls the size of the filesystem, the location of the kernel image, and other logistical matters. The Bootdisk_Contents file contains a list of the daemons, devices, directories, files, executable programs, libraries, and utilities that we explicitly wanted to include in the filesystem. The scripts that come with YARD automatically determine the external dependences of anything included, and add those to the filesystem before compressing the whole thing.

\subsubsection{NETBOOT}

We used Gero Kuhlmann's NETBOOT package ${ }^{8}$ to create the bootimage that each node downloads from the master computer. The bootimage is really just a concatenated copy of the Linux kernel (zImage.node) and the compressed root filesystem (root.gz). The NETBOOT software also includes a utility for creating a ROM image that is used to program the EPROMs in the ethernet card for each node. Although our ROM image was only $16 \mathrm{~kb}$, we used Am27C256 (32 kb) EPROMs because they were actually cheaper than the smaller chips.

\subsubsection{PVM}

The Parallel Virtual Machine (PVM) software ${ }^{9}$ allows a collection of computers connected by a network to cooperate on a problem as if they were a single multi-

\footnotetext{
${ }^{7}$ http://www.croftj.net/ fawcett/yard/

${ }^{8}$ http://www.han.de/ gero/netboot/

${ }^{9}$ http://www.epm.ornl.gov/pvm/
} 
processor parallel machine. It was developed in the 1990's at Oak Ridge National Laboratory (Geist et al., 1994). The software consists of a daemon running on each host in the virtual machine, and a library of routines that need to be incorporated into a computer program so that it can utilize all of the available computing power.

\subsection{How it works}

With the master computer up and running, we turn on one node at a time (to prevent the server from being overwhelmed by many simultaneous bootp requests). By default, the node tries to boot from the network first. It finds the bootrom on the ethernet card, and executes the ROM program. This program initializes the ethernet card and broadcasts a bootp request over the network.

When the server receives the request, it identifies the unique hardware address, assigns the corresponding IP address from the /etc/bootptab file, and allows the requesting node to download the bootimage. The node loads the Linux kernel image into memory, creates a 16 MB initial ramdisk, mounts the root filesystem, and starts all essential services and daemons.

Once all of the nodes are up, we start the PVM daemons on each node from the master computer. Any computer program that incorporates the PVM library routines and has been included in the root filesystem can then be run in parallel.

\subsection{Benchmarks}

Measuring the absolute performance of the metacomputer is difficult because the result strongly depends on the fraction of Floating-point Division operations (FDIVs) used in the benchmark code. Table 2.1 lists four different measures of the absolute speed in Millions of FLoating-point Operations Per Second (MFLOPS).

The code for MFLOPS(1) is essentially scalar, which means that it cannot exploit any advantages that are intrinsic to processor instruction sets; the percentage of FDIVs (9.6\%) is considered somewhat high. The code for MFLOPS(2) is fully vectorizable, which means that it can exploit advantages intrinsic to each processor, but the percentage of FDIVs (9.2\%) is still on the high side. The code 
Table 2.1: The Absolute Speed of the Metacomputer

\begin{tabular}{cccc}
\hline \hline Benchmark & P-II 300 MHz & K6-II $450 \mathrm{MHz}$ & Total Speed \\
\hline MFLOPS(1) & 80.6 & 65.1 & 4662.4 \\
MFLOPS(2) & 47.9 & 67.7 & 3699.2 \\
MFLOPS(3) & 56.8 & 106.9 & 7056.0 \\
MFLOPS(4) & 65.5 & 158.9 & 7180.8 \\
\hline \hline
\end{tabular}

for MFLOPS(3) is also fully vectorizable and the percentage of FDIVs (3.4\%) is considered moderate. The code for MFLOPS(4) is fully vectorizable, but the percentage of FDIVs is zero. We feel that MFLOPS(3) provides the best measure of the expected performance for the white dwarf code because of the moderate percentage of FDIVs. Adopting this value, we have achieved a price to performance ratio near $\$ 3.50 / \mathrm{MFLOPS}$.

The relative speed of the metacomputer is easy to measure. We simply compare the amount of time required to compute a specified number of white dwarf models using all 64 nodes to the amount of time required to calculate the same number of models using only one of the nodes. We find that the metacomputer is about 60 times faster than a single node by itself.

\section{7 $\quad$ Stumbling Blocks}

After more than 3 months without incident, one of the nodes abruptly died. One of the graduate students working in our lab reported, "One of your babies is crying!" As it turned out, the power supply had gone bad, frying the motherboard and the CPU fan. The processor overheated, shut itself off, and triggered an alarm. We now keep a few spare CPU fans and power supplies on hand. This is the only real problem we have had with the system, and it was easily fixed.

Since the first incident, this scenario has repeated itself five times over a three year period. This implies that such events can be expected at the rate of 2 per year for this many nodes. In addition to the more serious failures, there have been ten other power supply failures which did not result in peripheral hardware damage. The rate for these failures is $3-4$ per year. 


\section{Chapter 3}

\section{Parallel Genetic Algorithm}

"Evolution is cleverer than you are."

-Francis Crick

\subsection{Background}

The problem of extracting useful information from a set of observational data often reduces to finding the set of parameters for some theoretical model which results in the closest match to the observations. If the physical basis of the model is both accurate and complete, then the values of the parameters for the best-fit model can yield important insights into the nature of the object under investigation.

When searching for the best-fit set of parameters, the most fundamental consideration is: where to begin? Models of all but the simplest physical systems are typically non-linear, so finding the least-squares fit to the data requires an initial guess for each parameter. Generally, some iterative procedure is used to improve upon this first guess in order to find the model with the absolute minimum residuals in the multi-dimensional parameter-space.

There are at least two potential problems with this standard approach to model fitting. The initial set of parameters is typically determined by drawing upon the past experience of the person who is fitting the model. This subjective method is particularly disturbing when combined with a local approach to iter- 
ative improvement. Many optimization schemes, such as differential corrections (Proctor \& Linnell, 1972) or the simplex method (Kallrath \& Linnell, 1987), yield final results which depend to some extent on the initial guesses. The consequences of this sort of behavior are not serious if the parameter-space is well behaved - that is, if it contains a single, well defined minimum. If the parameterspace contains many local minima, then it can be more difficult for the traditional approach to find the global minimum.

\subsection{Genetic Algorithms}

An optimization scheme based on a genetic algorithm (GA) can avoid the problems inherent in more traditional approaches. Restrictions on the range of the parameter-space are imposed only by observations and by the physics of the model. Although the parameter-space so-defined is often quite large, the GA provides a relatively efficient means of searching globally for the best-fit model. While it is difficult for GAs to find precise values for the set of best-fit parameters, they are well suited to search for the region of parameter-space that contains the global minimum. In this sense, the GA is an objective means of obtaining a good first guess for a more traditional method which can narrow in on the precise values and uncertainties of the best-fit.

The underlying ideas for genetic algorithms were inspired by Charles Darwin's (1859) notion of biological evolution through natural selection. The basic idea is to solve an optimization problem by evolving the best solution from an initial set of completely random guesses. The theoretical model provides the framework within which the evolution takes place, and the individual parameters controlling it serve as the genetic building blocks. Observations provide the selection pressure. A comprehensive description of how to incorporate these ideas in a computational setting was written by Goldberg (1989).

Initially, the parameter-space is filled uniformly with trials consisting of randomly chosen values for each parameter, within a range based on the physics that the parameter is supposed to describe. The model is evaluated for each trial, and the result is compared to the observed data and assigned a fitness based on the relative quality of the match. A new generation of trials is then 
created by selecting from this population at random, weighted by the fitness.

To apply genetic operations to the new generation of trials, their characteristics must be encoded in some manner. The most straightforward way of encoding them is to convert the numerical values of the parameters into a long string of numbers. This string is analogous to a chromosome, and each number represents a gene. For example, a two parameter trial with numerical values $x_{1}=1.234$ and $y_{1}=5.678$ would be encoded into a single string of numbers ' 12345678 '.

Next, the encoded trials are paired up and modified in order to explore new regions of parameter-space. Without this step, the final solution could ultimately be no better than the single best trial contained in the initial population. The two basic operations are crossover which emulates sexual reproduction, and mutation which emulates happenstance and cosmic rays.

As an example, suppose that the encoded trial above is paired up with another trial having $x_{2}=2.468$ and $y_{2}=3.579$, which encodes to the string ' 24683579 '. The crossover procedure chooses a random position between two numbers along the string, and swaps the two strings from that position to the end. So if the third position is chosen, the strings become

$$
\begin{aligned}
& 123|45678 \rightarrow 123| 83579 \\
& 246|83579 \rightarrow 246| 45678
\end{aligned}
$$

Although there is a high probability of crossover, this operation is not applied to all of the pairs. This helps to keep favorable characteristics from being eliminated or corrupted too hastily. To this same end, the rate of mutation is assigned a relatively low probability. This operation allows for the spontaneous transformation of any particular position on the string into a new randomly chosen value. So if the mutation operation were applied to the sixth position of the second trial, the result might be

$$
24645|6| 78 \rightarrow 24645|0| 78
$$

After these operations have been applied, the strings are decoded back into sets of numerical values for the parameters. In this example, the new first string ' 12383579 ' becomes $x_{1}=1.238$ and $y_{1}=3.579$ and the new second string '24645078' becomes $x_{2}=2.464$ and $y_{2}=5.078$. This new generation replaces 
the old one, and the process begins again. The evolution continues until one region of parameter-space remains populated while other regions become essentially empty. The robustness of the solution can be established by running the GA several times with different random initialization.

Genetic algorithms have been used a great deal for optimization problems in other fields, but until recently they have not attracted much attention in astronomy. The application of GAs to problems of astronomical interest was promoted by Charbonneau (1995), who demonstrated the technique by fitting the rotation curves of galaxies, a multiply-periodic signal, and a magneto-hydrodynamic wind model. Many other applications of GAs to astronomical problems have appeared in the recent literature. Hakala (1995) optimized the accretion stream map of an eclipsing polar. Lang (1995) developed an optimum set of image selection criteria for detecting high-energy gamma rays. Kennelly et al. (1995) used radial velocity observations to identify the oscillation modes of a $\delta$ Scuti star. Lazio (1997) searched pulsar timing signals for the signatures of planetary companions. Charbonneau et al. (1998) performed a helioseismic inversion to constrain solar core rotation. Wahde (1998) determined the orbital parameters of interacting galaxies. Metcalfe (1999) used a GA to fit the light curves of an eclipsing binary star. The applicability of GAs to such a wide range of astronomical problems is a testament to their versatility.

\subsection{Parallelizing PIKAIA}

There are only two ways to make a computer program run faster - either make the code more efficient, or run it on a faster machine. We made a few design improvements to the original white dwarf code, but they decreased the runtime by only $\sim 10 \%$. We decided that we really needed access to a faster machine. We looked into the supercomputing facilities available through the university, but the idea of using a supercomputer didn't appeal to us very much; the process seemed to involve a great deal of red tape, and we weren't certain that we could justify time on a supercomputer in any case. To be practical, the GA-based fitting technique required a dedicated instrument to perform the calculations. We designed and configured such an instrument - an isolated network of 64 minimal 
PCs running Linux (Metcalfe \& Nather, 1999, 2000). To allow the white dwarf code to be run on this metacomputer, we incorporated the message passing routines of the Parallel Virtual Machine (PVM) software into the public-domain genetic algorithm PIKAIA.

\subsubsection{Parallel Virtual Machine}

The PVM software (Geist et al., 1994) allows a collection of networked computers to cooperate on a problem as if they were a single multi-processor parallel machine. All of the software and documentation was free. We had no trouble installing it, and the sample programs that came with the distribution made it easy to learn how to use. The trickiest part of the whole procedure was figuring out how to split up the workload among the various computers.

The GA-based fitting procedure for the white dwarf code quite naturally divided into two basic functions: evolving and pulsating white dwarf models, and manipulating the results from each generation of trials. When we profiled the distribution of execution time for each part of the code, this division became even more obvious. The majority of the computing time was spent evolving the starter model to a specific temperature. The GA is concerned only with collecting and organizing the results of many of these models, so it seemed reasonable to allocate many slave computers to carry out the model calculations while a master computer took care of the GA-related tasks.

In addition to decomposing the function of the code, a further division based on the data was also possible. Since there were many trials in each generation, the data required by the GA could easily be split into small, computationally manageable units. One model could be sent to each available slave computer, so the number of machines available would control the number of models which could be calculated at the same time.

One minor caveat to the decomposition of the data into separate models to be calculated by different computers is the fact that half of the machines are slightly faster than the other half. Much of the potential increase in efficiency from this parallelizing scheme could be lost if fast machines are not sent more models to compute than slow ones. This may seem trivial, but there is no mechanism 
built in to the current version of the PVM software to handle this procedure automatically.

It is also potentially problematic to send out new jobs only after receiving the results of previous jobs because the computers sometimes hang or crash. Again, this may seem obvious - but unless specifically asked to check, PVM cannot tell the difference between a crashed computer and one that simply takes a long time to compute a model. At the end of a generation of trials, if the master process has not received the results from one of the slave jobs, it would normally just continue to wait for the response indefinitely.

\subsubsection{The PIKAIA Subroutine}

PIKAIA is a self-contained, genetic-algorithm-based optimization subroutine developed by Paul Charbonneau and Barry Knapp at the High Altitude Observatory in Boulder, Colorado. Most optimization techniques work to minimize a quantity - like the root-mean-square (r.m.s.) residuals; but it is more natural for a genetic algorithm to maximize a quantity — natural selection works through survival of the fittest. So PIKAIA maximizes a specified FORTRAN function through a call in the body of the main program.

Unlike many GA packages available commercially or in the public domain, PIKAIA uses decimal (rather than binary) encoding. Binary operations are usually carried out through platform-dependent functions in FORTRAN, which makes it more difficult to port the code between the Intel and Sun platforms.

PIKAIA incorporates only the two basic genetic operators: uniform one-point crossover, and uniform one-point mutation. The mutation rate can be dynamically adjusted during the evolution, using either the linear distance in parameterspace or the difference in fitness between the best and median solutions in the population. The practice of keeping the best solution from each generation is called elitism, and is a default option in PIKAIA. Selection is based on ranking rather than absolute fitness, and makes use of the Roulette Wheel algorithm. There are three different reproduction plans available in PIKAIA: Steady-StateDelete-Random, Steady-State-Delete-Worst, and Full Generational Replacement. Only the last of these is easily parallelizable. 


\subsection{Master Program}

Starting with an improved unreleased version of PIKAIA, we incorporated the message passing routines of PVM into a parallel fitness evaluation subroutine. The original code evaluated the fitnesses of the population of trials one at a time in a DO loop. We replaced this procedure with a single call to a new subroutine that evaluates the fitnesses in parallel on all available processors.

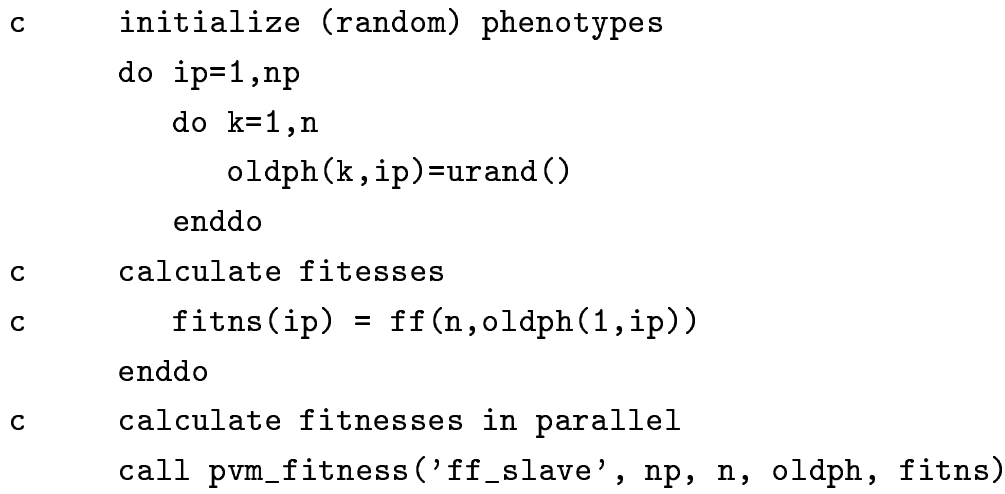

The parallel version of PIKAIA constitutes the master program which runs on Darwin, the central computer in the network. A full listing of the parallel fitness evaluation subroutine (PVM_FITNESS.F) is included in Appendix C. A flow chart for this code is shown in Figure 3.1.

After starting the slave program on every available processor (64 for our metacomputer), PVM_FITNESS.F sends an array containing the values of the parameters to each slave job over the network. In the first generation of the GA, these values are completely random; in subsequent generations, they are the result of the selection and mutation of the previous generation, performed by the non-parallel portions of PIKAIA.

Next, the subroutine listens for responses from the network and sends a new set of parameters to each slave job as it finishes the previous calculation. When all sets of parameters have been sent out, the subroutine begins looking for jobs that seem to have crashed and re-submits them to slaves that have finished and would otherwise sit idle. If a few jobs do not return a fitness after about five times the average runtime required to compute a model, the subroutine assigns them a fitness of zero. When every set of parameters in the generation have been assigned a fitness value, the subroutine returns to the main program to perform the genetic operations resulting in a new generation of models to calculate. The 


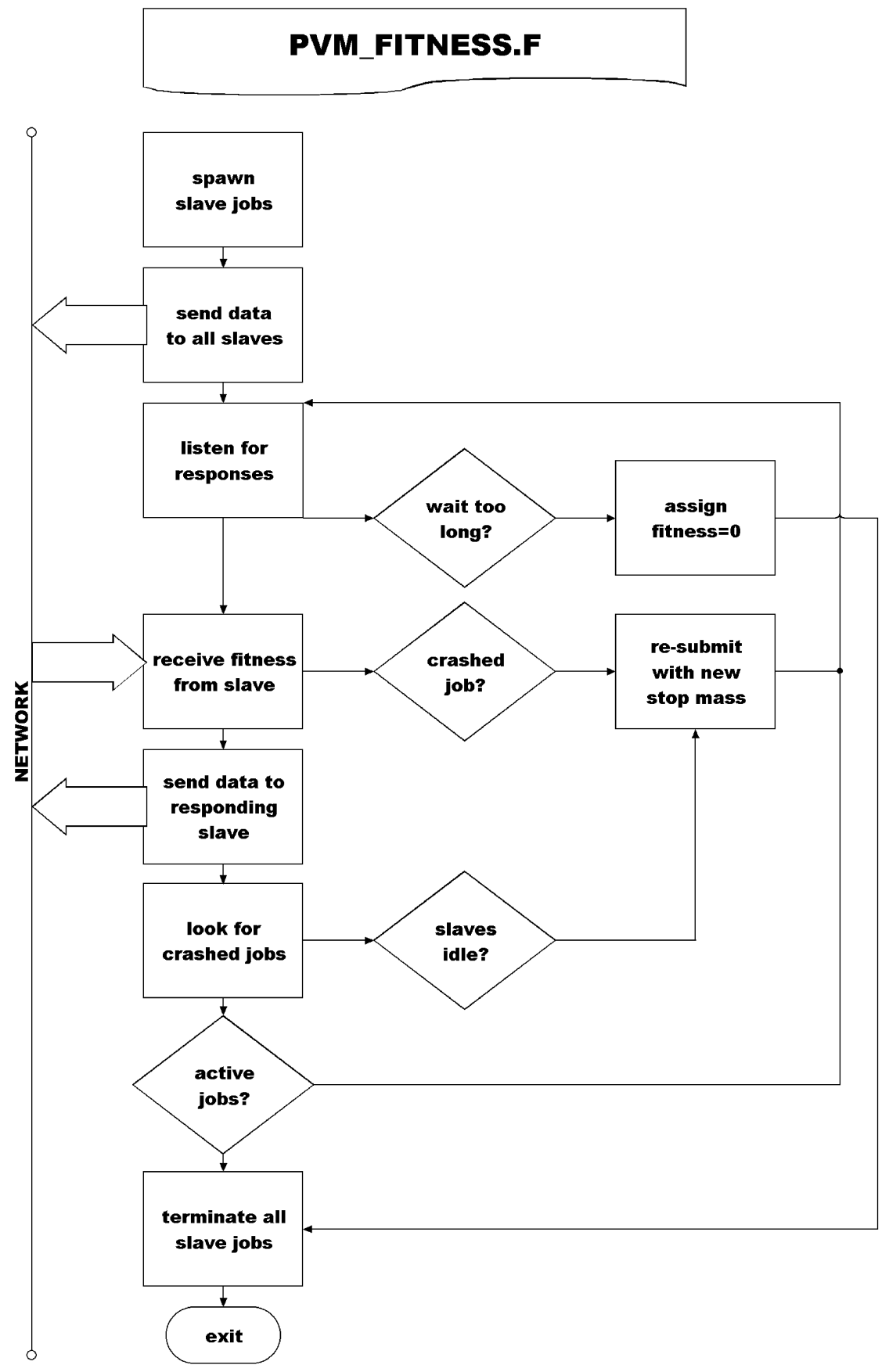

Figure 3.1: Flow chart for the parallel fitness evaluation subroutine, which runs on the master computer. 
process continues for a fixed number of generations, chosen to maximize the efficiency of the search. The optimal number of generations is determined by applying the method to a test problem with a known solution.

\subsection{Slave Program}

The original white dwarf code came in three pieces: (1) the evolution code, which evolves a starter model to a specific temperature, (2) the prep code, which converts the output of the evolution code into a different format, and (3) the pulsation code, which uses the output of the prep code to determine the pulsation periods of the model.

To get the white dwarf code to run in an automated way, we merged the three components of the original code into a single program, and added a front end that communicated with the master program through PVM routines. This

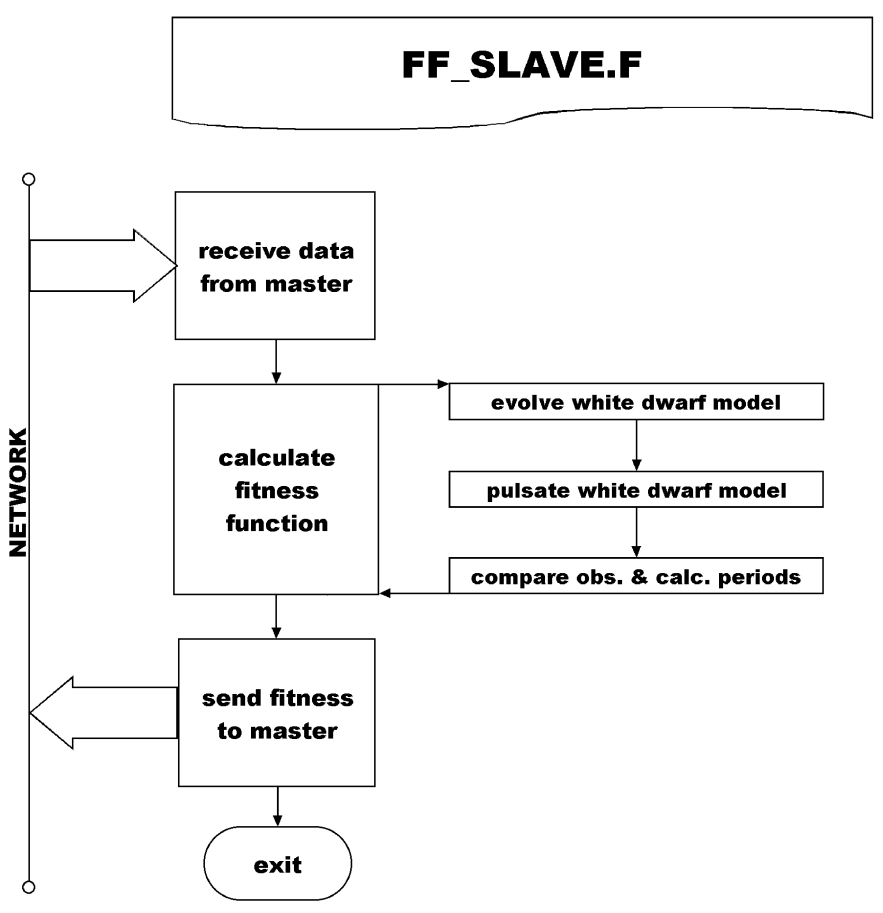

Figure 3.2: Flow chart for the slave program of the parallel code, which runs on each of the 64 nodes of the metacomputer. 
code (FF_SLAVE.F) constitutes the slave program, and is run on each node of the metacomputer. A full listing of this code is included in Appendix C, and a flow chart is shown in Figure 3.2.

The operation of the slave program is relatively simple. Once it is started by the master program, it receives a set of parameters from the network. It then calls the fitness function (the white dwarf code) with these parameters as arguments. The fitness function evolves a white dwarf model with characteristics specified by the parameters, determines the pulsation periods of this model, and then compares the calculated periods to the observed periods of a real white dwarf. A fitness based on how well the two sets of periods match is returned to the main program, which sends it to the master program over the network. The node is then ready to run the slave program again and receive a new set of parameters from the master program. 


\section{Chapter 4}

\section{Forward Modeling}

"Why do we always find a lost screwdriver in the last place we look?"

- Joe Wampler

\subsection{Introduction}

Having developed the hardware and software for the genetic-algorithm-based approach to model fitting, we were finally ready to learn something about white dwarf stars. There are presently three known classes of pulsating white dwarfs. The hottest class are the planetary nebula nucleus variables (PNNVs), which have atmospheres of ionized helium and are also called DOVs. These objects require detailed calculations that evolve a main sequence stellar model to the pre-white dwarf phase to yield accurate pulsation periods. The two cooler classes are the helium-atmosphere variable (DBV) and hydrogen-atmosphere variable (DAV) white dwarfs. The pulsation periods of these objects can be calculated accurately by evolving simpler, less detailed models called polytropes. The DAV stars are generally modeled as a core of carbon and oxygen with an overlying blanket of helium covered by a thin layer of hydrogen on the surface. The DBV stars are the simplest of all, with no detectable hydrogen - only a helium layer surrounding the carbon/oxygen core. In the spirit of solving the easier problem first, we decided to apply the GA method to the DBV star GD 358. 


\subsection{The DBV White Dwarf GD 358}

During a survey of eighty-six suspected white dwarf stars in the Lowell GD lists, Greenstein (1969) classified GD 358 as a helium atmosphere (DB) white dwarf based on its spectrum. Photometric $U B V$ and $u b v y$ colors were later determined by Bern \& Wramdemark (1973) and Wegner (1979) respectively. Time-series photometry by Winget, Robinson, Nather \& Fontaine (1982) revealed the star to be a pulsating variable - the first confirmation of a new class of variable (DBV) white dwarfs predicted by Winget (1981).

In May 1990, GD 358 was the target of a coordinated observing run with the Whole Earth Telescope (WET; Nather et al., 1990). The results of these observations were reported by Winget et al. (1994), and the theoretical interpretation was given in a companion paper by Bradley \& Winget (1994b). They found a series of nearly equally-spaced periods in the power spectrum which they interpreted as non-radial $g$-mode pulsations of consecutive radial overtone. They attempted to match the observed periods and the period spacing for these modes using earlier versions of the same theoretical models we have used in this analysis (see §4.3). Their optimization method involved computing a grid of models near a first guess determined from general scaling arguments and analytical relations developed by Kawaler (1990), Kawaler \& Weiss (1990), Brassard et al. (1992), and Bradley, Winget \& Wood (1993).

\subsection{DBV White Dwarf Models}

\subsubsection{Defining the Parameter-Space}

The most important parameters affecting the pulsation properties of DBV white dwarf models are the total stellar mass $\left(M_{*}\right)$, the effective temperature $\left(T_{\text {eff }}\right)$, and the mass of the atmospheric helium layer $\left(M_{\mathrm{He}}\right)$. We wanted to be careful to avoid introducing any subjective bias into the best-fit determination simply by defining the range of the search too narrowly. For this reason, we specified the range for each parameter based only on the physics of the model, and on observational constraints. 
The distribution of masses for isolated white dwarf stars, generally inferred from measurements of $\log g$, is strongly peaked near $0.6 M_{\odot}$ with a full width at half maximum (FWHM) of about $0.1 M_{\odot}$ (Napiwotzki, Green \& Saffer, 1999). Isolated main sequence stars with masses near the limit for helium ignition produce $\mathrm{C} / \mathrm{O}$ cores more massive than about $0.45 M_{\odot}$, so white dwarfs with masses below this limit must have helium cores (Sweigart, Greggio \& Renzini, 1990; Napiwotzki, Green \& Saffer, 1999). However, the universe is not presently old enough to produce helium core white dwarfs through single star evolution. We confine our search to masses between $0.45 M_{\odot}$ and $0.95 M_{\odot}$. Although some white dwarfs are known to be more massive than the upper limit of our search, these represent a very small fraction of the total population and, for reasonable assumptions about the mass-radius relation, all known DBVs appear to have masses within the range of our search (Beauchamp et al., 1999).

The span of temperatures within which DB white dwarfs are pulsationally unstable is known as the DB instability strip. The precise location of this strip is the subject of some debate, primarily because of difficulties in matching the temperature scales from ultraviolet and optical spectroscopy and the possibility of hiding trace amounts of hydrogen in the envelope (Beauchamp et al., 1999). The most recent temperature determinations for the 8 known DBV stars were done by Beauchamp et al. (1999). These measurements, depending on various assumptions, place the red edge as low as $21,800 \mathrm{~K}$, and the blue edge as high as $27,800 \mathrm{~K}$. Our search includes all temperatures between 20,000 K and 30,000 K.

The mass of the atmospheric helium layer must not be greater than about $10^{-2} M_{*}$ or the pressure of the overlying material would theoretically initiate helium burning at the base of the envelope. At the other extreme, none of our models pulsate for helium layer masses less than about $10^{-8} M_{*}$ over the entire temperature range we are considering (Bradley \& Winget, 1994a). The practical limit is actually slightly larger than this theoretical limit, and is a function of mass. For the most massive white dwarfs we consider, our models run smoothly with a helium layer as thin as $5 \times 10^{-8} M_{*}$, while for the least massive the limit is $4 \times 10^{-7} M_{*}$ (see Figure 4.1 ). 


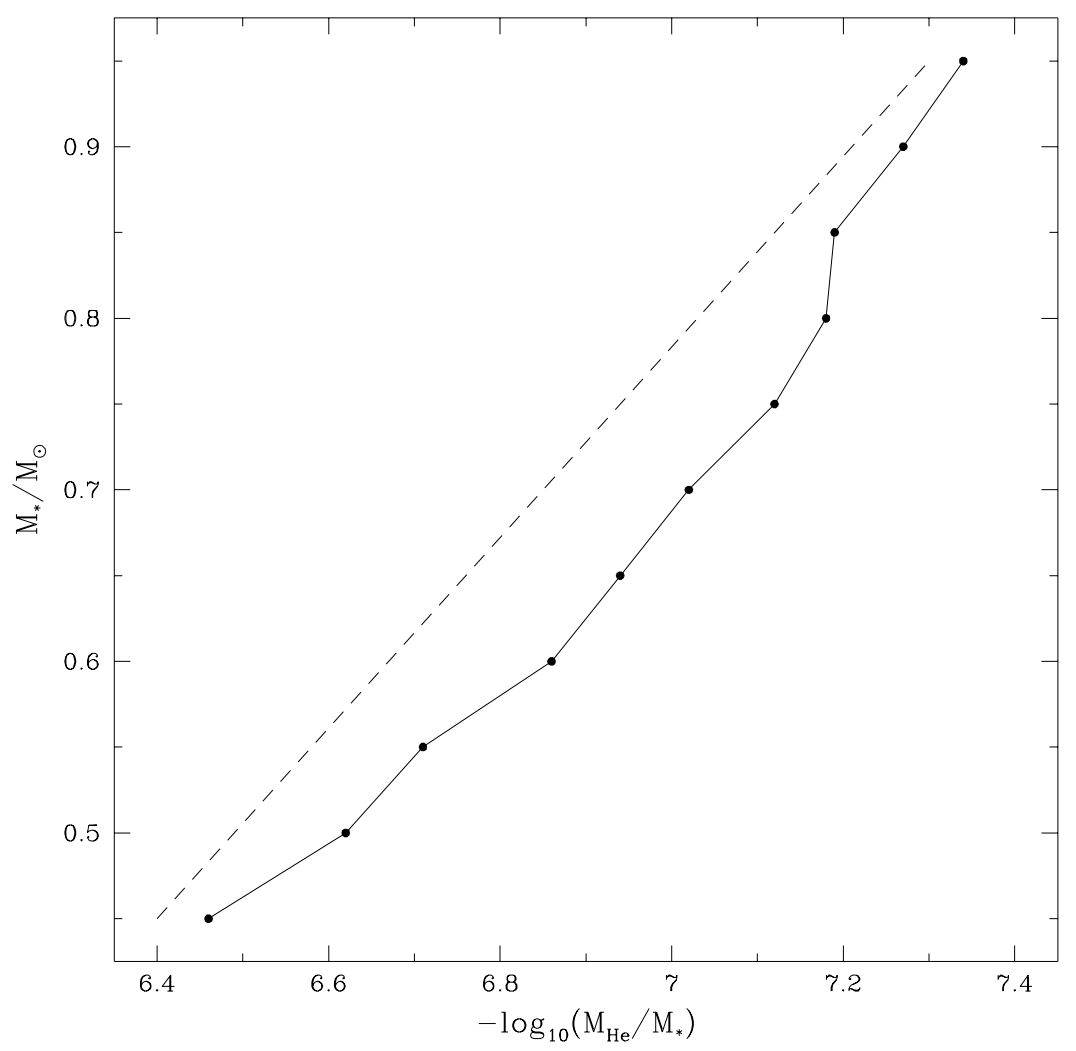

Figure 4.1: The numerical thin limit of the fractional helium layer mass for various values of the total mass (connected points) and the linear cut implemented in the WD-40 code (dashed line).

\subsubsection{Theoretical Models}

To find the theoretical pulsation modes of a white dwarf, we start with a static model of a pre-white dwarf and allow it to evolve quasi-statically until it reaches the desired temperature. We then calculate the adiabatic non-radial oscillation frequencies for the output model. The initial 'starter' models can come from detailed calculations that evolve a main-sequence star all the way to its prewhite dwarf phase, but this is generally only important for accurate models of the hot DO white dwarfs. For the cooler DB and DA white dwarfs, it is sufficient to start with a hot polytrope of order $2 / 3$ (i.e. $P \propto \rho^{5 / 3}$ ). The cooling tracks of these polytropes converge with those of the pre-white dwarf models well above the 
temperatures at which DB and DA white dwarfs are observed to be pulsationally unstable (Wood, 1990).

To allow fitting for the total mass, we generated a grid of 100 starter models with masses between 0.45 and $0.95 M_{\odot}$. The entire grid originated from a $0.6 M_{\odot}$ carbon-core polytrope starter model. We performed a homology transform on this model to generate three new masses: $0.65,0.75$, and $0.85 M_{\odot}$. We relaxed each of these three, and then used all four to generate the grid. All models with masses below $0.6 M_{\odot}$ were generated by a direct homology transform of the original $0.6 M_{\odot}$ polytrope. For masses between $0.605 \rightarrow 0.745 M_{\odot}$ and from $0.755 \rightarrow 0.895 M_{\odot}$, we used homology transforms of the relaxed $0.65 M_{\odot}$ and $0.75 M_{\odot}$ models respectively. The models with masses greater than $0.9 M_{\odot}$ were homology transforms of the relaxed $0.85 M_{\odot}$ model.

To evolve a starter model to a specific temperature, we used the White Dwarf Evolution Code (WDEC) described in detail by Lamb \& Van Horn (1975) and by Wood (1990). This code was originally written by Martin Schwarzschild, and has subsequently been updated and modified by many others including: Kutter \& Savedoff (1969), Lamb \& Van Horn (1975), Winget (1981), Kawaler (1986), Wood (1990), Bradley (1993), and Montgomery (1998). The equation of state (EOS) for the cores of our models come from Lamb (1974), and from Fontaine, Graboske \& Van Horn (1977) for the envelopes. We use the updated OPAL opacity tables from Iglesias \& Rogers (1993), neutrino rates from Itoh et al. (1996), and the ML3 mixing-length prescription of Böhm \& Cassinelli (1971). The evolution calculations for the core are fully self-consistent, but the envelope is treated separately. The core and envelope are stitched together and the envelope is adjusted to match the boundary conditions at the interface. Adjusting the helium layer mass involves stitching an envelope with the desired thickness onto the core before starting the evolution. Because this is done while the model is still very hot, there is plenty of time to reach equilibrium before the model approaches the final temperature.

We determined the pulsation frequencies of the output models using the adiabatic non-radial oscillation (ANRO) code described by Kawaler (1986), originally written by Carl Hansen, which solves the pulsation equations using the RungeKutta-Fehlberg method. 
We have made extensive practical modifications to these programs, primarily to allow models to be calculated without any intervention by the user. The result is a combined evolution/pulsation code that runs smoothly over a wide range of input parameters. We call this new code WD-40. Given a mass, temperature, and helium layer mass within the ranges discussed above, WD-40 will evolve and pulsate the specified white dwarf model and return a list of the theoretical pulsation periods.

\subsection{Model Fitting}

Using the parallel version of PIKAIA on our metacomputer, we fixed the population size at 128 trials, and initially allowed the GA to run for 250 generations. We used 2-digit decimal encoding for each of the three parameters, which resulted in a temperature resolution of $100 \mathrm{~K}$, a mass resolution of $0.005 M_{\odot}$, and a resolution for the helium layer thickness of 0.05 dex. The uniform single-point crossover probability was fixed at $85 \%$, and the mutation rate was allowed to vary between $0.1 \%$ and $16.6 \%$, depending on the linear distance in parameter-space between the trials with the median and the best fitnesses.

\subsubsection{Application to Noiseless Simulated Data}

To quantify the efficiency of our method for this problem, we used the WD-40 code to calculate the pulsation periods of a model within the search space, and then attempted to find the set of input parameters $\left[T_{\text {eff }}=25,000 \mathrm{~K}, M_{*}=0.600\right.$ $\left.M_{\odot}, \log \left(M_{\mathrm{He}} / M_{*}\right)=-5.96\right]$ using the GA. We performed 20 independent runs using different random initialization each time. The first order solutions found in each case by the GA are listed in Table 4.1. In 9 of the 20 runs, the GA found the exact set of input parameters, and in 4 other runs it finished in a region of parameter-space close enough for a small (1331 point) grid to reveal the exact answer. Since none of the successful runs converged between generations 200 and 250, we stopped future runs after 200 generations.

From the 13 runs that converged in 200 generations, we deduce an efficiency for the method (GA + small grid) of $\sim 65 \%$. This implies that the probability 
Table 4.1: Results for Noiseless Simulated Data

\begin{tabular}{cccccc}
\hline \hline & \multicolumn{3}{c}{ First-Order Solution } & \multicolumn{2}{c}{ Generation } \\
\cline { 2 - 5 } Run & $T_{\text {eff }}$ & $M_{*} / M_{\odot}$ & $\log \left(M_{\mathrm{He}} / M_{*}\right)$ & r.m.s. & Found \\
\hline 01 & 26,800 & 0.560 & -5.70 & 0.67 & 245 \\
02 & 25,000 & 0.600 & -5.96 & 0.00 & 159 \\
03 & 24,800 & 0.605 & -5.96 & 0.52 & 145 \\
04 & 25,000 & 0.600 & -5.96 & 0.00 & 68 \\
05 & 22,500 & 0.660 & -6.33 & 1.11 & 97 \\
06 & 25,000 & 0.600 & -5.96 & 0.00 & 142 \\
07 & 25,000 & 0.600 & -5.96 & 0.00 & 97 \\
08 & 25,000 & 0.600 & -5.96 & 0.00 & 194 \\
09 & 25,200 & 0.595 & -5.91 & 0.42 & 116 \\
10 & 26,100 & 0.575 & -5.80 & 0.54 & 87 \\
11 & 23,900 & 0.625 & -6.12 & 0.79 & 79 \\
12 & 25,000 & 0.600 & -5.96 & 0.00 & 165 \\
13 & 26,100 & 0.575 & -5.80 & 0.54 & 92 \\
14 & 25,000 & 0.600 & -5.96 & 0.00 & 95 \\
15 & 24,800 & 0.605 & -5.96 & 0.52 & 42 \\
16 & 26,600 & 0.565 & -5.70 & 0.72 & 246 \\
17 & 24,800 & 0.605 & -5.96 & 0.52 & 180 \\
18 & 25,000 & 0.600 & -5.96 & 0.00 & 62 \\
19 & 24,100 & 0.620 & -6.07 & 0.76 & 228 \\
20 & 25,000 & 0.600 & -5.96 & 0.00 & 167 \\
\hline \hline
\end{tabular}

of missing the correct answer in a single run is $\sim 35 \%$. By running the GA several times, we reduce the probability of not finding the correct answer: the probability that two runs will both be wrong is $\sim 12 \%$, for three runs it is $\sim 4 \%$, and so on. Thus, to reduce the probability of not finding the correct answer to below $1 \%$ we need to run the GA, on average, 5 times. For 200 generations of 128 trials, this requires $\sim 10^{5}$ model evaluations. By comparison, an exhaustive search of the parameter-space with the same resolution would require $10^{6}$ model evaluations, so our method is comparably global but about $10 \times$ more efficient than an exhaustive search of parameter-space. Even with this efficiency and our ability to run the models in parallel, each run of the GA required about 6 hours to complete. 


\subsubsection{The Effect of Gaussian Noise}

Having established that the GA could find the correct answer for noiseless data, we wanted to see how noise on the frequencies might affect it. Before adding noise to the input frequencies, we attempted to characterize the actual noise present on frequencies determined from a WET campaign. We approached this problem in two different ways.

First, we tried to characterize the noise empirically by looking at the differences between the observed and predicted linear combination frequencies. When we take the Fourier Transform of the long light curves from WET observations, we are effectively decomposing the signal into perfect sinusoidal components of various frequencies. The actual light variations arising from a single pulsation mode are generally not perfect sinusoids, and the amplitude often varies on relatively short timescales. This leads to significant power in the Fourier Transform at integer multiples and fractions of the real pulsation frequency. These so-called harmonics and sub-harmonics are examples of linear combination frequencies, but they are not nearly as prevalent as the combinations that arise from the interaction of different pulsation modes in the observed light curves.

When two pulsation modes with different frequencies $f_{1}$ and $f_{2}$ are present, the resulting light curve shows oscillations at the sum of the two frequencies $f_{1}+f_{2}$. As the frequencies go into and out of phase or "beat" with each other, the amplitude of the light variation grows and shrinks in a periodic manner at a frequency equal to the difference of the two components $f_{1}-f_{2}$. If the amplitude of the beating is smaller than expected due to some non-linear effect, then the Fourier Transform will reveal power not only at the real pulsation frequencies, but also at these combination frequencies. If we can properly identify the pulsation modes that produce a specific linear combination, it allows us to estimate the uncertainty on our frequency measurements because the combination should show up at a precise sum or difference.

We used the mode identifications of Vuille et al. (2000) from the 1994 WET run on GD 358. There were a total of 63 combinations identified: 20 sum and 11 difference frequencies of 2-mode combinations, 30 sum and difference 3-mode combinations, and 2 combinations involving 4 modes. We used the measured frequencies of the parent modes to predict the frequency of each linear combination, 
and then compared this to the observed frequency. The distribution of observed minus computed frequencies for these 63 modes, and the best-fit Gaussian is shown in the top panel of Figure 4.2. The distribution has $\sigma=0.17 \mu \mathrm{Hz}$.

Second, we tried to characterize the noise by performing the standard analysis for WET runs on many simulated light curves to look at the distribution of differences between the input and output frequencies. We generated 100 synthetic GD 358 light curves using the 57 observed frequencies and amplitudes from Winget et al. (1994). Each light curve had the same time span as the 1990 WET run (965,060 seconds) sampled with the same interval (every 10 seconds) but
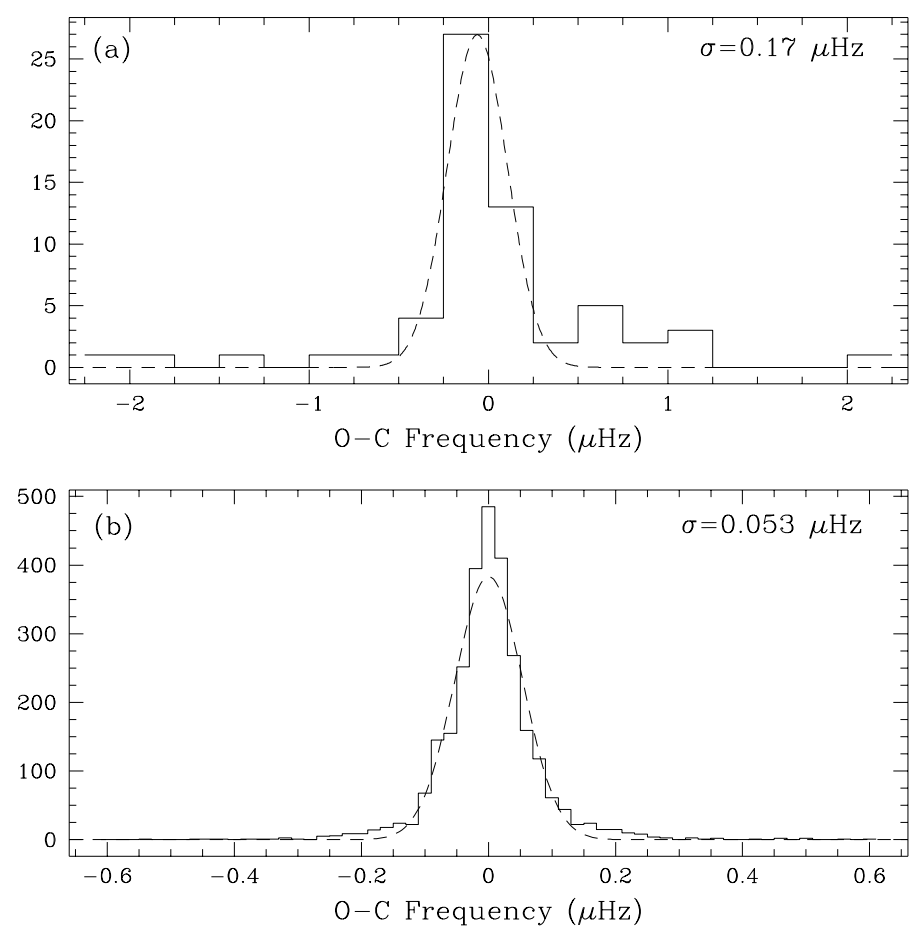

Figure 4.2: The distribution of differences between (a) the observed and predicted frequencies of linear combination modes identified by Vuille et al. (2000) in the 1994 WET run on GD 358 and the best-fit Gaussian with $\sigma=0.17 \mu \mathrm{Hz}$ (dashed line) and (b) the input and output frequencies for the 11 modes used to model GD 358 from simulated WET runs (see $\S 4.4 .2$ for details) and the best-fit Gaussian with $\sigma=0.053 \mu \mathrm{Hz}$ (dashed line). 
without any gaps in coverage. Although the noise in the observed light curves was clearly time-correlated, we found that the distribution around the mean light level for the comparison star after the standard reduction procedure was well represented by a Gaussian. So we added Gaussian noise to the simulated light curves to yield a signal-to-noise ratio $S / N \approx 2$, which is typical of the observed data. We took the discrete Fourier Transform of each light curve, and identified peaks in the same way as is done for real WET runs. We calculated the differences between the input frequencies and those determined from the simulation for the 11 modes used in the seismological analysis by Bradley \& Winget (1994b). The distribution of these differences is shown in the bottom panel of Figure 4.2, along with the best-fit Gaussian which has $\sigma=0.053 \mu \mathrm{Hz}$. Because the noise measurement from linear combination frequencies suffered from low-number statistics, we adopted the noise estimate from the synthetic light curves for our studies of the effect of noise on the GA method.

Using the same input model as in $§ 4.4 .1$, we added random offsets drawn from a Gaussian distribution with $\sigma=0.053 \mu \mathrm{Hz}$ to each of the frequencies. We produced 10 sets of target frequencies from 10 unique realizations of the noise, and then applied the GA method to each set. In all cases the best of 5 runs of the GA found the exact set of parameters from the original input model, or a region close enough to reveal the exact solution after calculating the small grid around the first guess. To reassure ourselves that the success of the GA did not depend strongly on the amount of noise added to the frequencies, we also performed fits for several realizations of the larger noise estimate from the analysis of linear combination frequencies. The method always succeeded in finding the original input model.

\subsubsection{Application to GD 358}

Having thoroughly characterized the GA method, we finally applied it to real data. We used the same 11 periods used by Bradley \& Winget (1994b). As in their analysis, we assumed that the periods were consecutive radial overtones and that they were all $\ell=1$ modes (Winget et al., 1994, give detailed arguments to support this assumption). Anticipating that the GA might have more difficulty with non-synthetic data, we decided to perform a total of 10 GA runs for each 
core composition. This should reduce the chances of not finding the best answer to less than about 3 in 10,000 .

To facilitate comparison with previous results, we obtained fits for six different combinations of core composition and internal chemical profiles: pure $\mathrm{C}$, pure $\mathrm{O}$, and both "steep" and "shallow" internal chemical profiles for 50:50 and 20:80 C/O cores (see Bradley, Winget \& Wood, 1993; Bradley \& Winget, 1994b).

We also ran the GA with an alternate fitness criterion for the 50:50 C/O "steep" case, which contains the best-fit model of Bradley \& Winget (1994b). Normally, the GA only attempts to match the pulsation periods. We reprogrammed it to match both the periods and the period spacing, which was the fitness criterion used by Bradley \& Winget. Within the range of parameters they considered, using this alternate fitness criterion, the GA found best-fit model parameters consistent with Bradley \& Winget's solution.

\subsection{Initial Results}

The general features of the 3-dimensional parameter-space for GD 358 are illustrated in Figure 4.3. All combinations of parameters found by the GA for a 50:50 $\mathrm{C} / \mathrm{O}$ steep core having r.m.s. period differences smaller than 3 seconds are shown as square points in this plot. The two panels are orthogonal projections of the search space, so each point in the left panel corresponds one-to-one with a point in the right panel. Essentially, Figure 4.3 shows which combinations of model parameters yield reasonably good matches to the periods observed in GD 358 for this core composition.

The most obvious feature of the parameter-space is the presence of more than one region that yields a good match to the observations. Generally, the good fits seem to cluster in two families corresponding to thick and thin helium layers. This is the first exciting result of applying the GA method. The best-fit solution of Bradley \& Winget (1994b) falls in the family with thin helium layers. This solution was problematic at the time because the earlier asteroseismological investigation of the hot white dwarf PG 1159-035 (Winget et al., 1991) implied that it had a relatively thick helium layer. If there is an evolutionary connection between PG 1159 stars and the DBVs, it was more difficult to understand if the helium layers were significantly different. If a better solution for GD 358 exists 


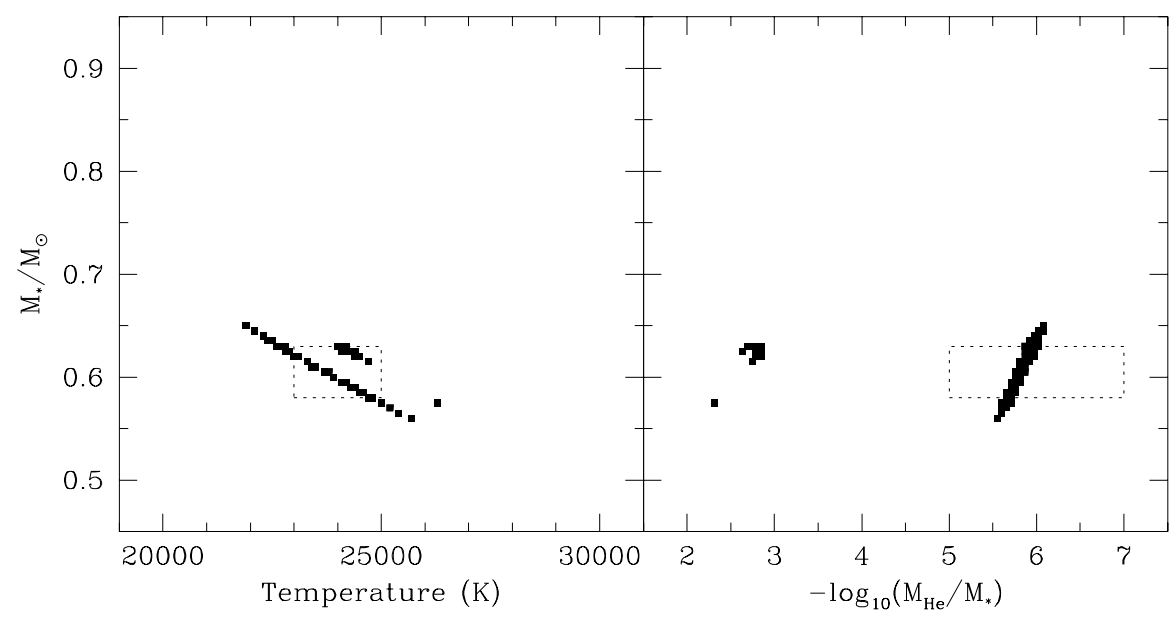

Figure 4.3: Front and side views of the GA search space for a C/O 50:50 core with a "steep" internal chemical profile. Square points mark the locations of every model found by the GA with an r.m.s. deviation smaller than 3 seconds for the periods observed in GD 358. The dashed line shows the range of parameters considered by Bradley \& Winget (1994b).

among the family with thick helium layers, this long-standing controversy might be resolved.

The other obvious features in Figure 4.3 are the parameter-correlations in both projections, causing the good fits to fall along lines in parameter-space rather than on a single point. The correlation between total mass and fractional helium layer mass is relatively easy to understand. Brassard et al. (1992) showed that the pulsation periods of trapped modes in white dwarf models are strongly influenced by the scaled location of the composition transition zone. They developed an expression showing that these periods are directly proportional to the fractional radius of the composition interface. As the total mass of a white dwarf increases, the surface area decreases, so the mass of helium required to keep the interface at the same fractional radius also decreases. Thus, a thinner helium layer can compensate for an overestimate of the mass.

The correlation between mass and temperature is slightly more complicated. The natural frequency that dominates the determination of white dwarf model pulsation frequencies is the Brunt-Väisälä frequency (named after meteorologists 
David Brunt and Yuri Väisälä) which reflects the difference between the actual and the adiabatic density gradients. As the temperature decreases, the matter becomes more degenerate, so the Brunt-Väisälä frequency in much of the star tends to zero. The pulsation periods of a white dwarf model in some sense reflect the average of the Brunt-Väisälä frequency throughout the star, so a decrease in temperature leads to lower pulsation frequencies. Higher mass models have higher densities, which generally lead to higher pulsation frequencies. So an overestimate of the mass can compensate for the effect of an underestimate of the temperature.

The results for all six core compositions and internal chemical profiles are shown in Figure 4.4, where we have used color to indicate the absolute quality of
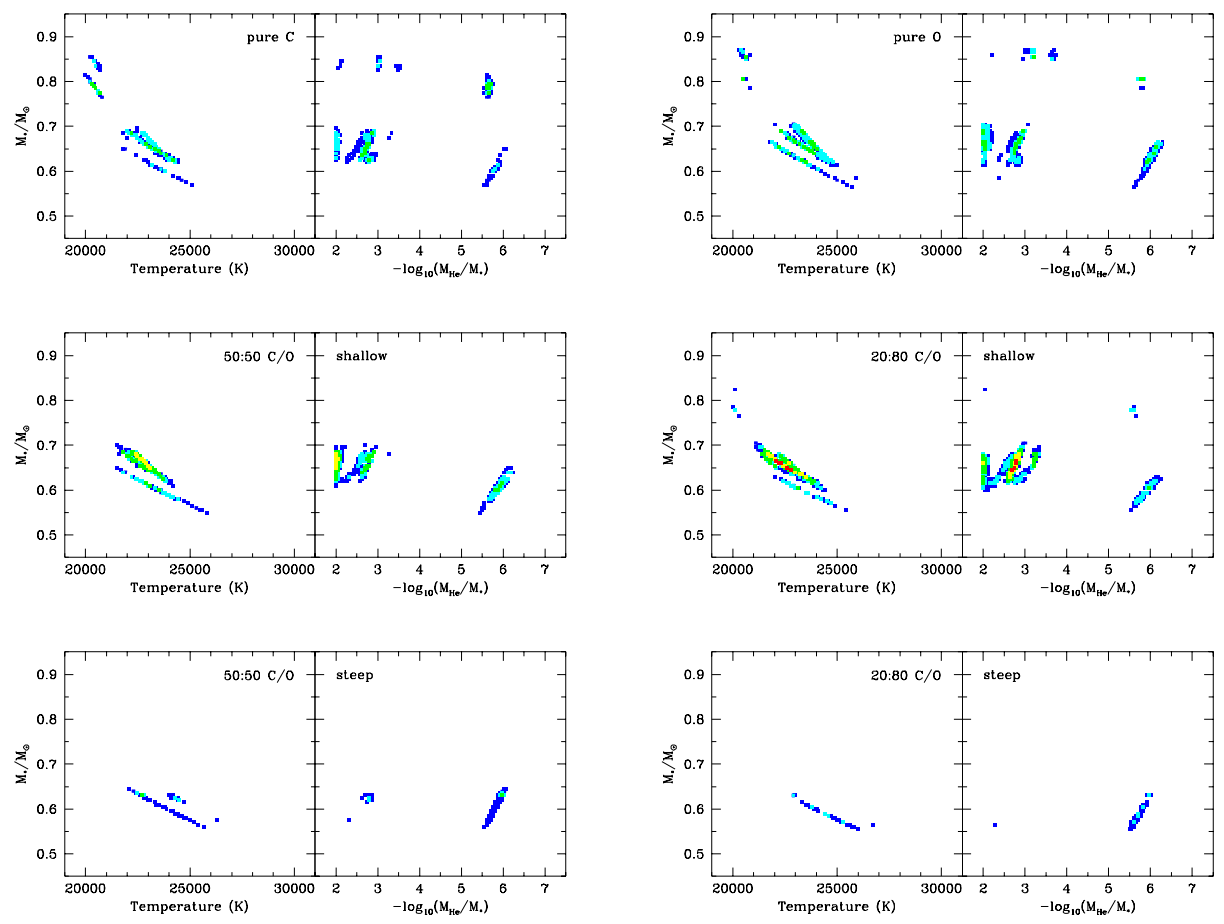

Figure 4.4: The families of models found by the GA which yield good matches to the periods observed in GD 358 for various core compositions and internal chemical profiles. The quality of the fit is indicated by the color of the square: r.m.s. $<3.0$ seconds (blue),$<2.75$ seconds (cyan), $<2.5$ seconds (green) $<2.0$ seconds (yellow), and $<1.65$ seconds (red). 
each fit. We find that reasonable fits are possible with every core composition, but excellent fits (indicated by red points in the figure) are only possible for a specific core composition and internal chemical profile. Pure $\mathrm{C}$ and pure $\mathrm{O}$ models appear to have more families of possible solutions, but the high-mass families have luminosities that can be ruled out based on the observed parallax of GD 358 (Harrington et al., 1985). Mixed C/O cores generally seem to produce better fits, but internal chemical profiles that are steep are much worse than those that are shallow. Among the mixed $\mathrm{C} / \mathrm{O}$ cores with shallow internal chemical profiles, the 20:80 mix produces the best fits of all.

The parameters for the best-fit models and measures of their absolute quality are listed in Table 4.2. For each core composition, the best-fit for both the thick and thin helium layer families are shown. As indicated, several fits can be ruled

Table 4.2: Results for GD 358 Data

\begin{tabular}{|c|c|c|c|c|}
\hline \multirow[b]{2}{*}{ Core Properties } & \multicolumn{3}{|c|}{ Best Models } & \multirow[b]{2}{*}{ r.m.s. } \\
\hline & $T_{\text {eff }}$ & $M / M_{\odot}$ & $\log \left(M_{\mathrm{He}} / M_{*}\right)$ & \\
\hline \multirow[t]{2}{*}{ pure $\mathrm{C}$} & 20,300 & 0.795 & -5.66 & $2.17^{a}$ \\
\hline & 23,100 & 0.655 & -2.74 & 2.30 \\
\hline \multirow[t]{2}{*}{ 50:50 C/O "shallow". } & 22,800 & 0.665 & -2.00 & 1.76 \\
\hline & 23,100 & 0.610 & -5.92 & 2.46 \\
\hline \multirow[t]{2}{*}{ 50:50 C/O "steep" } & 22,700 & 0.630 & -5.97 & 2.42 \\
\hline & 24,300 & 0.625 & -2.79 & 2.71 \\
\hline \multirow[t]{2}{*}{ 20:80 C/O "shallow". } & 22,600 & 0.650 & -2.74 & $1.50^{b}$ \\
\hline & 23,100 & 0.605 & -5.97 & 2.48 \\
\hline \multirow{2}{*}{ 20:80 C/O "steep" } & 22,900 & 0.630 & -5.97 & 2.69 \\
\hline & 27,300 & 0.545 & -2.16 & $2.87^{a}$ \\
\hline \multirow[t]{2}{*}{ pure $\mathrm{O}$} & 20,500 & 0.805 & -5.76 & $2.14^{a}$ \\
\hline & 23,400 & 0.655 & -2.79 & 2.31 \\
\hline
\end{tabular}

${ }^{a}$ Luminosity is inconsistent with observations

${ }^{b}$ Best-fit solution 
out based on their luminosities. Our new best-fit model for GD 358 has a mass and temperature marginally consistent with those inferred from spectroscopy.

\subsection{Internal Composition \& Structure}

The initial 3-parameter fits to GD 358 make it clear that both the central oxygen abundance and the shape of the internal chemical profile should be treated as free parameters. We modified our code to allow any central oxygen mass fraction $\left(X_{\mathrm{O}}\right)$ between 0.00 and 1.00 with a resolution of 1 percent. To explore different chemical profiles we fixed $X_{\mathrm{O}}$ to its central value out to a fractional mass parameter $(q)$ which varied between 0.10 and 0.85 with a resolution of 0.75 percent. From this point, we forced $X_{\mathrm{O}}$ to decrease linearly in mass to zero oxygen at the 95 percent mass point.

This parameterization is a generalized form of the "steep" and "shallow" profiles. We used these profiles so that our results could be easily compared to earlier work by Wood (1990) and Bradley, Winget \& Wood (1993). The latter authors define both profiles in their Figure 1. The "shallow" profile corresponds approximately to $q=0.5$, and "steep" corresponds roughly to $q=0.8$. However, in our generalized parameterization we have moved the point where the oxygen abundance goes to zero from a fractional mass of 0.9 out to a fractional mass of 0.95. This coincides with the boundary in our models between the self-consistent core and the envelope, where we describe the $\mathrm{He} / \mathrm{C}$ transition using a diffusion equilibrium profile from the method of Arcoragi \& Fontaine (1980) with diffusion exponents of \pm 3 . We do not presently include oxygen in the envelopes, so the mass fraction of oxygen must drop to zero by this point.

We calculated the magnitude of deviations from the mean period spacing for models using our profiles compared to those due to smooth profiles from recent theoretical calculations by Salaris et al. (1997). The smooth theoretical profiles caused significantly larger deviations, so we conclude that the abrupt changes in the oxygen abundance resulting from our parameterization do not have an unusually large effect on the period spacing. Although the actual chemical profiles will almost certainly differ from the profiles resulting from our simple parameterization, we should still be able to probe the gross features of the interior structure 
by matching one or another linear region of the presumably more complicated physical profiles.

We used the same ranges and resolution for $M_{*}, T_{\text {eff }}$, and $M_{\mathrm{He}}$ as in the initial study, so the search space for this 5-parameter problem is 10,000 times larger than for the 3-parameter case. Initially we tried to vary all 5 parameters simultaneously, but this proved to be impractical because of the parameter-correlation between $M_{*}$ and $q$. The pulsation properties of the models depend on the radial location in the chemical profile where the oxygen mass fraction begins to change. If the GA finds a combination of $M_{*}$ and $q$ that yields a reasonably good fit to the data, most changes to either one of them by itself will not improve the fit. As a consequence, simultaneous changes to both parameters are required to find a better fit, and since this is not very probable the GA must run for a very long time. Tests on synthetic data for the full 5-parameter problem yielded only a 10 percent probability of finding the input model even when we ran for 2000 generations - ten times longer than for the 3-parameter case. By contrast, when we used a fixed value of $q$ and repeated the test with only 4 free parameters, the GA found the input model in only 400 generations for 8 out of 10 runs. Even better, by fixing the mass and allowing $q$ to vary, it took only 250 generations to find the input model in 7 out of 10 runs. This suggests that it might be more efficient to alternate between these two subsets of 4 parameters, fixing the fifth parameter each time to its best-fit value from the previous iteration, until the results of both fits are identical.

Since we do not know a priori the precise mass of the white dwarf, we need to ensure that this iterative 4-parameter approach will work even when the mass is initially fixed at an incorrect value. To test this, we calculated the pulsation periods of the best-fit $0.65 M_{\odot}$ model for GD 358 from Table 4.2 and then iteratively applied the two 4-parameter fitting routines, starting with the mass fixed at $0.60 M_{\odot}$-an offset comparable to the discrepancy between the mass found in Table 4.2 and the value found by Bradley \& Winget (1994b). The series of fits leading to the input model are shown in Table 4.3. This method required only 3 iterations, and for each iteration we performed 10 runs with different random initialization to yield a probability of finding the best-fit much greater than 99.9 percent. In the end, the method required a total of $2.5 \times 10^{6}$ model evaluations 
Table 4.3: Convergence of the Method on Simulated Data

\begin{tabular}{cccccc}
\hline \hline Iteration & $T_{\text {eff }}$ & $M_{*} / M_{\odot}$ & $\log \left(M_{\mathrm{He}} / M_{*}\right)$ & $X_{\mathrm{O}}$ & $q$ \\
\hline $1^{a}$ & 23,600 & 0.600 & -5.76 & 0.52 & 0.55 \\
$1^{b}$ & 22,200 & 0.660 & -2.79 & 0.99 & 0.55 \\
$2^{a}$ & 22,200 & 0.660 & -2.79 & 0.88 & 0.51 \\
$2^{b}$ & 22,600 & 0.650 & -2.74 & 0.85 & 0.51 \\
$3^{a}$ & 22,600 & 0.650 & -2.74 & 0.80 & 0.50 \\
$3^{b}$ & 22,600 & 0.650 & -2.74 & 0.80 & 0.50 \\
\hline \hline
\end{tabular}

${ }^{a}$ Value of $M_{*} / M_{\odot}$ fixed

${ }^{b}$ Value of $q$ fixed.

(128 trials per generation, 10 runs of 650 generations per iteration). This is about 200 times more efficient than calculating the full grid in each iteration, and about 4,000 times more efficient than a grid of the entire 5-dimensional space.

Next, we applied this iterative 4-parameter method to the observed pulsation periods of GD 358. We initially fixed the mass at $0.61 M_{\odot}$, the value inferred from the original asteroseismological study by Bradley \& Winget (1994b). The solution converged after four iterations, and the best-fit values of the five parameters were:

$$
\begin{array}{rlrl}
T_{\text {eff }} & =22,600 \mathrm{~K} & X_{\mathrm{O}} & =0.84 \\
M_{*} / M_{\odot} & =0.650 & q & =0.49 \\
\log \left(M_{\mathrm{He}} / M_{*}\right) & =-2.74 & &
\end{array}
$$

Note that the values of $M_{*}, T_{\text {eff }}$ and $M_{\mathrm{He}}$ are identical to the best-fit in Table 4.2. The best-fit mass and temperature still differ significantly from the values inferred from spectroscopy by Beauchamp et al. (1999). However, the luminosity of our best-fit model is consistent with the luminosity derived from the measured parallax of GD 358 (Harrington et al., 1985).

To alleviate any doubt that the GA had found the best combination of $X_{\mathrm{O}}$ and $q$, and to obtain a more accurate estimate of the uncertainties on these parameters, we calculated a grid of 10,000 models with the mass, temperature, and helium layer mass fixed at their best-fit values. A contour plot of this grid near the solution found by the GA is shown in Figure 4.5. 


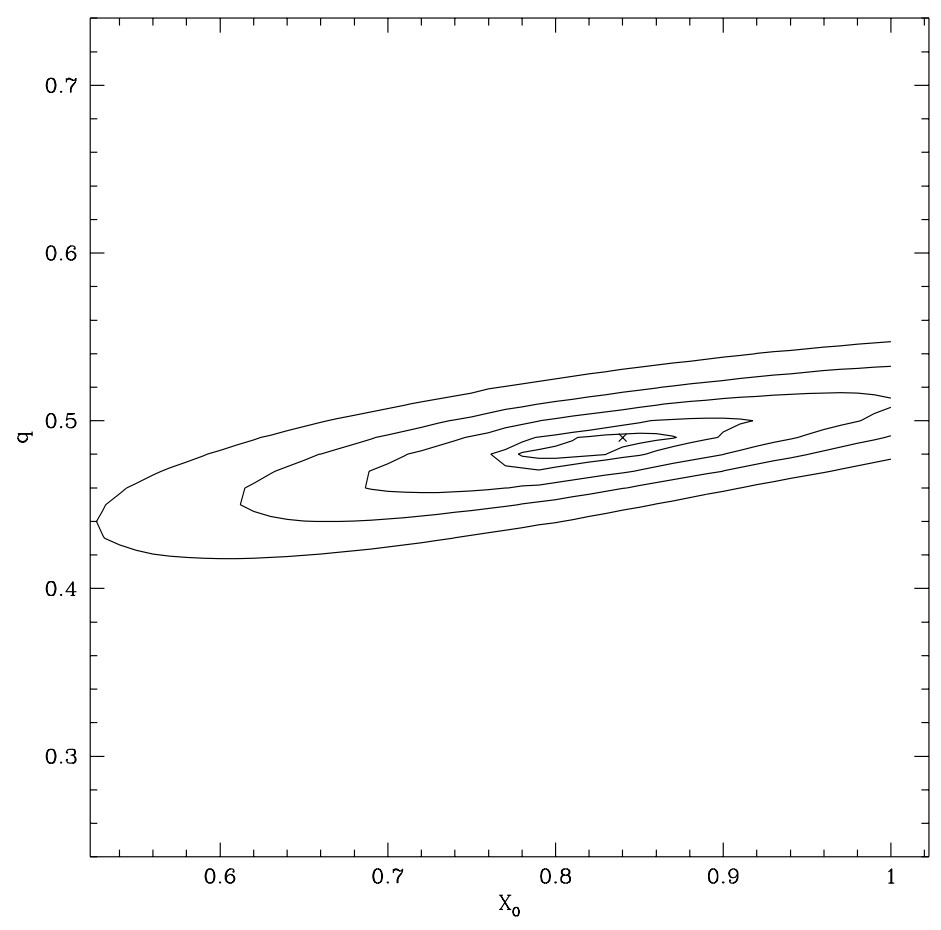

Figure 4.5: A contour plot of the central oxygen mass fraction $\left(X_{\mathrm{O}}\right)$ versus the fractional mass location of the change in the oxygen gradient $(q)$, with $M_{*}, T_{\text {eff }}$, and $M_{\mathrm{He}} / M_{*}$ fixed at their best-fit values. The model with the absolute minimum residuals (identical to the best-fit found by the GA) is marked with an X. The contours are drawn at 1, 3, 10, 25 and 40 times the observational noise.

\subsection{Constraints on Nuclear Physics}

During carbon burning in a red giant star, the triple- $\alpha$ process competes with the ${ }^{12} \mathrm{C}(\alpha, \gamma){ }^{16} \mathrm{O}$ reaction for the available $\alpha$-particles. As a consequence, the final ratio of carbon to oxygen in the core of a white dwarf is a measure of the relative cross-sections of these two reactions (Buchmann, 1996). The value of the ${ }^{12} \mathrm{C}(\alpha, \gamma){ }^{16} \mathrm{O}$ cross-section at stellar energies is presently the result of an extrapolation across eight orders of magnitude from laboratory data (Fowler, 1986). The triple- $\alpha$ reaction is relatively well determined at the relevant energies, so the constraint that comes from measuring the $\mathrm{C} / \mathrm{O}$ ratio in the core of a white dwarf is much more precise than other methods. 
Adopting the central oxygen mass fraction from the 5-parameter best-fit forward modeling ( $X_{\mathrm{O}}=84 \pm 3$ percent) we can place preliminary constraints on the ${ }^{12} \mathrm{C}(\alpha, \gamma){ }^{16} \mathrm{O}$ cross-section. Salaris et al. (1997) made detailed evolutionary calculations for main-sequence stellar models with masses between 1 and 7 $\mathrm{M}_{\odot}$ to provide internal chemical profiles for the resulting white dwarfs. For the bulk of the calculations they adopted the rate of Caughlan et al. (1985) for the ${ }^{12} \mathrm{C}(\alpha, \gamma){ }^{16} \mathrm{O}$ reaction $\left(S_{300}=240 \mathrm{keV}\right.$ barns $)$, but they also computed an evolutionary sequence using the lower cross-section inferred by Woosley, Timmes \& Weaver (1993) from solar abundances $\left(S_{300}=170 \mathrm{keV}\right.$ barns). The chemical profiles from both rates had the same general shape, but the oxygen abundances were uniformly smaller for the lower rate. In both cases the $\mathrm{C} / \mathrm{O}$ ratio was constant out to the 50 percent mass point, a region easily probed by white dwarf pulsations.

The central oxygen mass fraction is lower in higher mass white dwarf models. The rate of the triple- $\alpha$ reaction (a three-body process) increases faster at higher densities than does the ${ }^{12} \mathrm{C}(\alpha, \gamma){ }^{16} \mathrm{O}$ reaction. As a consequence, more helium is used up in the production of carbon, and relatively less is available to produce oxygen in higher mass models. Interpolating between the models of Salaris et al. (1997) which used the higher value of the cross-section, we expect a central oxygen mass fraction for a $M_{*}=0.65 M_{\odot}$ model of $X_{\mathrm{O}}^{\text {high }}=0.75$. Using additional calculations for the low rate (M. Salaris 2001, private communication), the expected value is $X_{\mathrm{O}}^{\text {low }}=0.62$. Extrapolating to the value inferred from our 5 -parameter forward modeling, we estimate that the astrophysical S-factor at 300 $\mathrm{keV}$ for the ${ }^{12} \mathrm{C}(\alpha, \gamma){ }^{16} \mathrm{O}$ cross-section is in the range $S_{300}=290 \pm 15 \mathrm{keV}$ barns (internal uncertainty only). 


\section{Chapter 5}

\section{Reverse Approach}

"The only real voyage of discovery consists not in seeking new landscapes but in having new eyes."

- Marcel Proust

\subsection{Introduction}

The results of forward modeling with one adjustable point in the chemical profile make it clear that information about the internal structure is contained in the data, and we just need to learn how to extract it. If we want to test more complicated adjustable profiles, forward modeling quickly becomes too computationally expensive as the dimensionality of the search space increases. We need to devise a more efficient approach to explore the myriad possibilities.

The natural frequency that dominates the determination of pulsation periods in white dwarf models is the Brunt-Väisälä (BV) frequency, which we calculate using the Modified Ledoux treatment described in Tassoul, Fontaine \& Winget (1990). To get a sense of how the pulsation periods depend on the BV frequency in various regions of the model interior, we added a smooth perturbation to the best-fit model of GD 358 from Table 4.2, moving it one shell at a time from the center to the surface. The perturbation artificially decreased the BV frequency across seven shells, with a maximum amplitude of 10 percent. We monitored the effect on each of the pulsation periods as the perturbation moved 
outward through the interior of the model. The results of this experiment for the pulsation periods corresponding to those observed in GD 358 are shown in Figure 5.1. Essentially, this experiment demonstrates that the pulsation periods are sensitive to the conditions all the way down to the inner few percent of the model. Since the observational uncertainties on each period are typically only a few hundredths of a second, even small changes to the BV frequency in the model interior are significant.

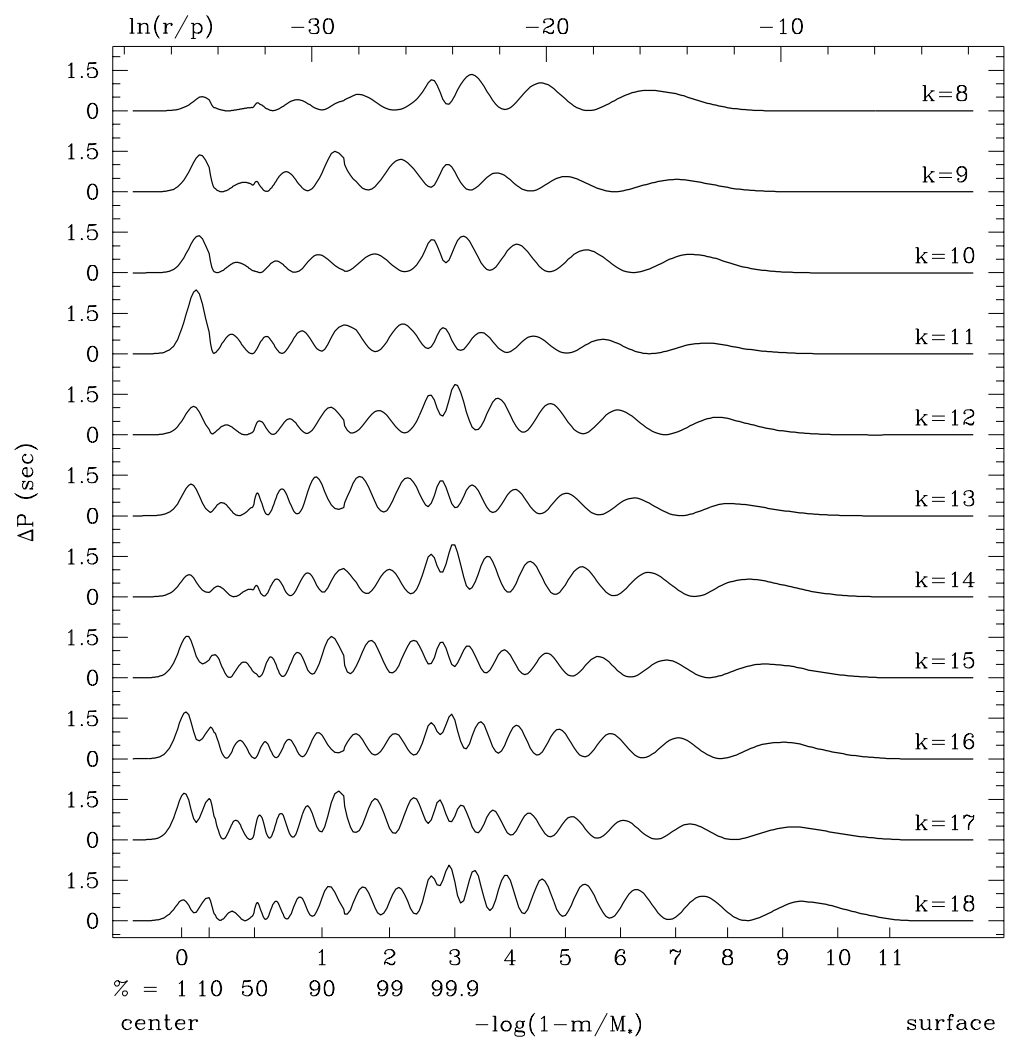

Figure 5.1: For the best-fit model of GD 358 from Table 4.2 this plot shows the change in pulsation period for $\ell=1$ modes of various radial overtone number $(k)$ which result from a smooth artificial $10 \%$ decrease in the Brunt-Väisälä frequency as a function of the natural log of the ratio of the distance from the center of the model to the local pressure (top axis) and the fractional mass coordinate $-\log \left(1-m / M_{*}\right)$ (bottom axis). The center of the model is to the left, and the surface is to the right. Also indicated is the mass fraction expressed as a percentage for several values closer to the center. 


\subsection{Model Perturbations}

The root-mean-square (r.m.s.) residuals between the observed pulsation periods in GD 358 and those calculated for the best-fit from forward modeling are still much larger than the observational noise. This suggests that either we have left something important out of our model, or we have neglected to optimize one or more of the parameters that could in principle yield a closer match to the observations. To investigate the latter possibility, we introduced ad hoc perturbations to the BV frequency of the best-fit model to see if the match could be improved. Initially, we concentrated on the region of the Brunt-Väisälä curve that corresponds to the internal chemical profile.

If we look at the BV frequency for models with the same mass, temperature, helium layer mass, and central oxygen mass fraction but different internal chemical profiles (see Figure 5.2) it becomes clear that the differences are localized. In general, we find that changes in the composition gradient cause shifts in the BV frequency. Moving across an interface where the gradient becomes steeper, the BV frequency shifts higher; at an interface where the gradient becomes more shallow, the BV frequency shifts lower. The greater the change in the gradient, the larger the shift in the BV frequency.

\subsubsection{Proof of Concept}

We began by generating a model with the same mass, temperature, helium layer mass, and internal composition as the best-fit from Table 4.2, but using a uniform internal chemical profile with constant 20:80 C/O out to the 95 percent mass point. We identified a sequence of 100 shells in this model spanning a range of fractional mass from 0.20 to 0.97 and perturbed the BV frequency to try to produce a better match to the observations. We parameterized the perturbation as a linearly varying multiplicative factor applied to the BV frequency over a range of shells, described by four parameters: (1) the innermost shell to perturb, (2) the magnitude of the perturbation at the innermost shell, (3) the number of shells in the perturbation range, and (4) the magnitude of the perturbation at the outermost shell. These 4 parameters are sufficient to describe a profile with two abrupt changes in the composition gradient. 

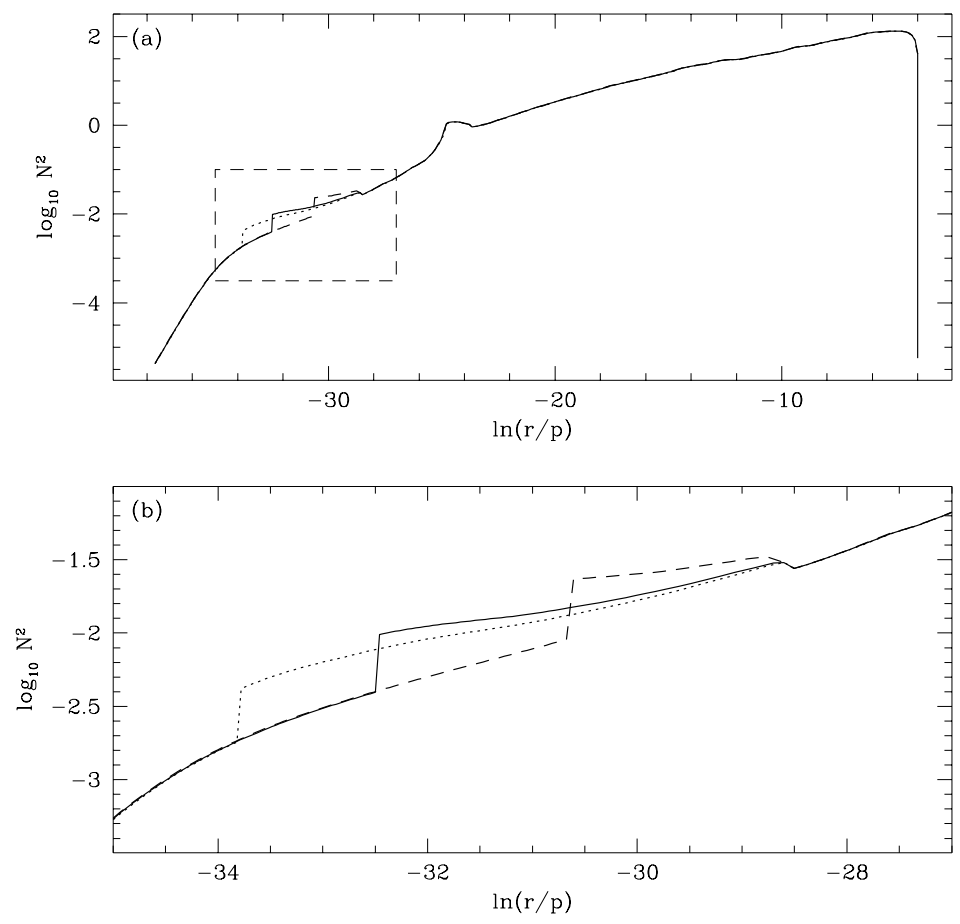

Figure 5.2: The Brunt-Väisälä frequency as a function of the radial coordinate $\ln (\mathrm{r} / \mathrm{p})$ for several models with the same mass, temperature, helium layer mass, and central oxygen mass fraction but different internal chemical profiles (a) from the center of the model at left to the surface at right, and (b) only in the range of $\ln (\mathrm{r} / \mathrm{p})$ indicated by the dashed box in the upper panel. The three curves correspond to a profile with $q$ equal to 0.2 (dotted), 0.49 (solid), and 0.8 (dashed).

The innermost shell was allowed to be any of the 100 shells, and the number of shells in the perturbation range was allowed to be between 0 and 100 . If the chosen range would introduce perturbations outside of the sequence of 100 shells, the outermost of these shells was the last to be perturbed. The magnitude of the perturbation was allowed to be a multiplicative factor between 1.0 and 3.0 at both the innermost and outermost perturbed shells, and was interpolated linearly in the shells between them.

By using a GA to optimize the perturbation, we can try many random possibilities and eventually find the global best-fit with far fewer model evaluations than a full grid search of the parameter-space. We demonstrated this by in- 
troducing a particular perturbation to the model and determining the pulsation periods. Using the same unperturbed model, we then attempted to find the parameters of the perturbation by matching the periods using the GA. In 9 out of 10 runs (500 generations of 64 trials), the GA found a first-order solution within two grid points of the input perturbation. Thus, the GA combined with a small (625 point) grid search yields a 90 percent probability of success for an individual run. By repeating the process several times, the probability of finding the correct answer quickly exceeds 99.9 percent, even while the number of model evaluations required remains hundreds of times lower than a full grid of the parameter-space.

\subsubsection{Application to GD 358}

Having demonstrated that the method works on calculated model periods, we applied it to the observed pulsation periods of GD 358. We began with a model similar to the 5-parameter best-fit determined from forward modeling, but again using a uniform internal chemical profile (constant 16:84 C/O out to $\left.0.95 \mathrm{~m} / M_{*}\right)$. After the GA had found the best-fit perturbation for GD 358, we reverse-engineered the corresponding chemical profile.

To accomplish this, we first looked in the unperturbed model for the fractional mass corresponding to the innermost and outermost shells in the perturbation range. We fixed the oxygen abundance to that of the unperturbed model from the center out to the fractional mass of the innermost perturbed shell. The size of the shift in the BV frequency is determined by how much the composition gradient changes at this point, so we adjusted the oxygen abundance at the fractional mass of the outermost perturbed shell until the change in the gradient produced the required shift. Finally, we fixed the oxygen abundance to that value from the outermost perturbed shell out to a fractional mass of 0.95 , where it abruptly goes to zero.

After we found the $\mathrm{C} / \mathrm{O}$ profile of the best-fit perturbation in this way, we fixed this reverse-engineered profile in the models and performed a new fit from forward modeling with the GA to re-optimize the mass, temperature, helium layer mass, and central oxygen mass fraction. The BV curve of the final model differs slightly, of course, from that of the original uniform composition model with the 
perturbation added. But the approximate internal structure is preserved, and leads to a better match to the observed pulsation periods than we could have otherwise found.

\subsection{Results}

The calculated periods and period spacings $\left(\Delta P=P_{k+1}-P_{k}\right)$ for the best-fit models from the 5-parameter forward modeling and from the reverse approach are shown in the bottom two panels of Figure 5.3 along with the data for GD 358 . The best-fit models of Bradley \& Winget (1994b) and Metcalfe, Nather \& Winget (2000) are shown in the top two panels for comparison. The data in Figure 5.3 for the observations and our best-fit models are given in Table 5.1. Some of the improvement evident in the panels of Figure 5.3 is certainly due to the fact that we have increased the number of free parameters. To evaluate whether or not the new models represent a significant improvement to the fit, we use the Bayes Information Criterion (BIC), following Montgomery, Metcalfe \& Winget (2001).

The fits listed in Table 4.2 used $n_{p}=3$ completely free parameters, sampling several combinations of two additional parameters. This amounts to a partial

Table 5.1: Periods and Period Spacings for GD 358 and Best-fit Models

\begin{tabular}{|c|c|c|c|c|c|c|c|c|}
\hline \multirow[b]{2}{*}{$k$} & \multicolumn{2}{|c|}{ Observed } & \multicolumn{2}{|c|}{ 3-par fit } & \multicolumn{2}{|c|}{ 5-par fit } & \multicolumn{2}{|c|}{ 7-par fit } \\
\hline & $P$ & $\Delta P$ & $P$ & $\Delta P$ & $\bar{P}$ & $\Delta P$ & $P$ & $\Delta P$ \\
\hline $08 \ldots$ & 423.27 & 40.96 & 422.31 & 42.26 & 422.36 & 40.92 & 422.75 & 39.69 \\
\hline 09 . & 464.23 & 37.36 & 464.57 & 36.77 & 463.28 & 38.01 & 462.43 & 37.46 \\
\hline 10. & 501.59 & 40.16 & 501.35 & 35.88 & 501.29 & 37.50 & 499.90 & 39.70 \\
\hline 11. & 541.75 & 35.01 & 537.23 & 39.04 & 538.79 & 36.77 & 539.60 & 36.65 \\
\hline 12 . & 576.76 & 41.52 & 576.27 & 42.79 & 575.56 & 43.33 & 576.25 & 42.10 \\
\hline 13. & 618.28 & 40.07 & 619.06 & 39.79 & 618.89 & 39.85 & 618.36 & 40.25 \\
\hline 14. & 658.35 & 42.29 & 658.85 & 42.97 & 658.74 & 42.36 & 658.61 & 42.90 \\
\hline $15 \ldots$ & 700.64 & 33.66 & 701.82 & 32.76 & 701.10 & 34.33 & 701.51 & 33.44 \\
\hline 16. & 734.30 & 36.37 & 734.58 & 36.92 & 735.42 & 36.99 & 734.95 & 36.59 \\
\hline 17. & 770.67 & 40.03 & 771.50 & 39.30 & 772.41 & 37.34 & 771.54 & 39.60 \\
\hline $18 \ldots$ & 810.7 & 44.1 & 810.80 & 44.34 & 809.75 & 44.63 & 811.14 & 44.15 \\
\hline
\end{tabular}




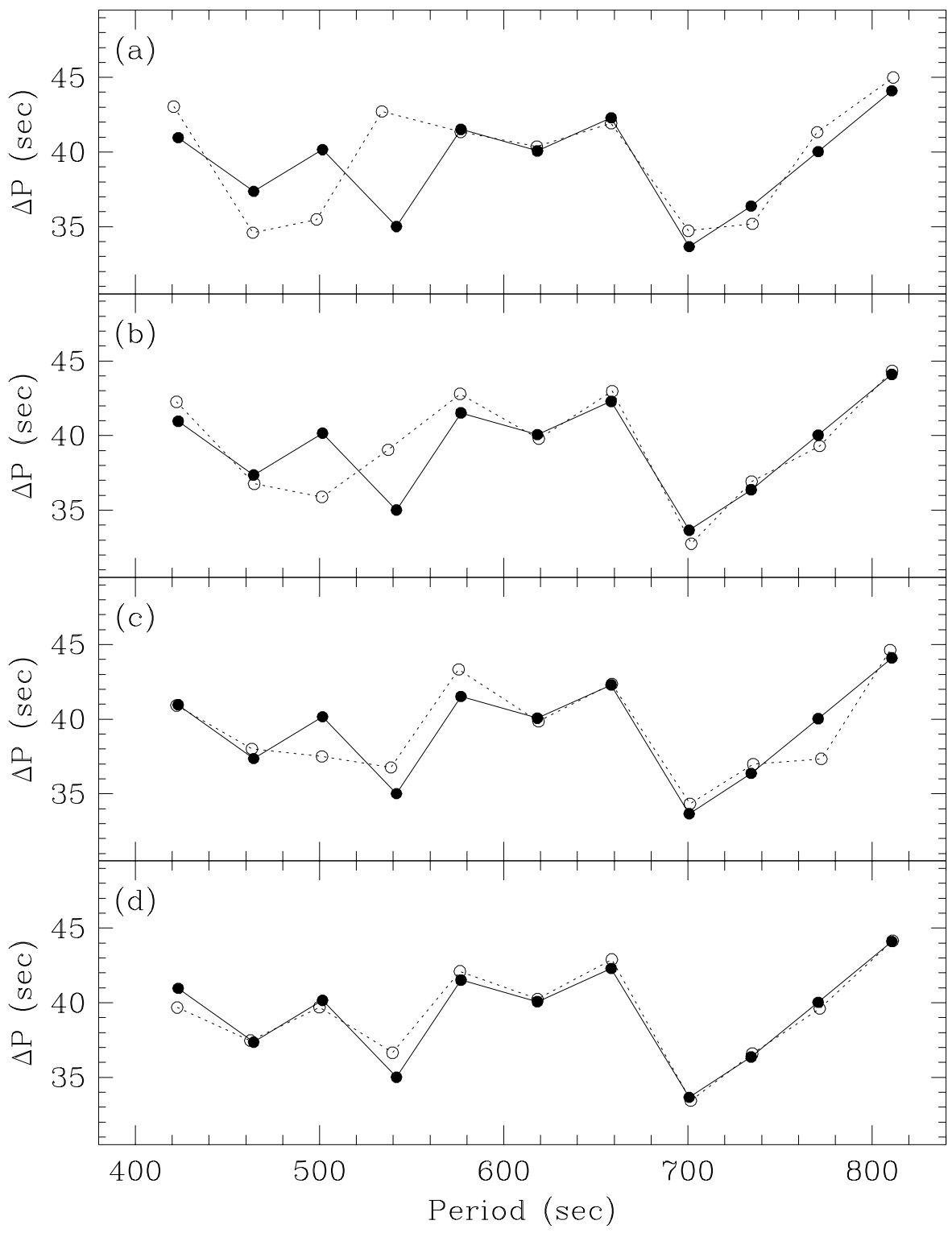

Figure 5.3: The periods and period spacings observed in GD 358 (solid points) with the theoretical best-fit models (open points) from (a) Bradley \& Winget (1994b), (b) Metcalfe, Nather \& Winget (2000), (c) the 5-parameter forward modeling presented in $\S 4.6$, and $(\mathrm{d})$ the reverse approach. Uncertainties on the observations are smaller than the size of the points in this figure. 
optimization in 5 dimensions. To make a fair comparison we use the best carboncore model from Table 4.2, which represents the best truly 3-parameter fit. This model had r.m.s. residuals of $\sigma(P)=2.30$ seconds for the periods and $\sigma(\Delta P)=$ 2.65 seconds for the period spacings. For $N=11$ data points, the BIC leads us to expect the residuals of a $n_{p}=5$ fit to decrease to $\sigma(P)=1.84$ and $\sigma(\Delta P)=2.13$ just from the addition of the extra parameters. In fact, the fit from the forward modeling presented in $\S 4.6$ has $\sigma(P)=1.28$ and $\sigma(\Delta P)=1.42$, so we conclude that the improvement is statistically significant.

The results of the reverse approach presented in $\S 5.2$ are harder to evaluate because we are perturbing the BV frequency directly, rather than through a specific parameter. We consider each additional point in the internal chemical profile where the composition gradient changes to be a free parameter. Under this definition, the perturbed models are equivalent to a 7-parameter fit since there are three such points in the profiles, compared to only one for the 5-parameter case. If we again use the BIC, we expect the residuals to decrease from their $n_{p}=5$ values to $\sigma(P)=1.03$ and $\sigma(\Delta P)=1.14$ seconds. After re-optimizing the other four parameters using the profile inferred from the reverse approach, the residuals actually decreased to $\sigma(P)=1.11$ and $\sigma(\Delta P)=0.71$ seconds. The decrease in the period residuals is not significant, but the period spacings are improved considerably. This is evident in the bottom panel of Figure 5.3.

\subsection{Chemical Profiles}

The internal chemical profiles corresponding to the best-fit models from the 5parameter forward modeling and from the reverse approach are shown in Figure 5.4 with the theoretical profile for a $0.61 M_{\odot}$ model from Salaris et al. (1997), scaled to a central oxygen mass fraction of 0.80 . The profile from the best-fit forward modeling matches the location and slope of the initial shallow decrease in the theoretical profile. The reverse approach also finds significant structure in this region of the model, and is qualitatively similar to the Salaris et al. (1997) profile to the extent that our parameterization allows. It is encouraging that both approaches agree with each other and bear some resemblance to the models. 

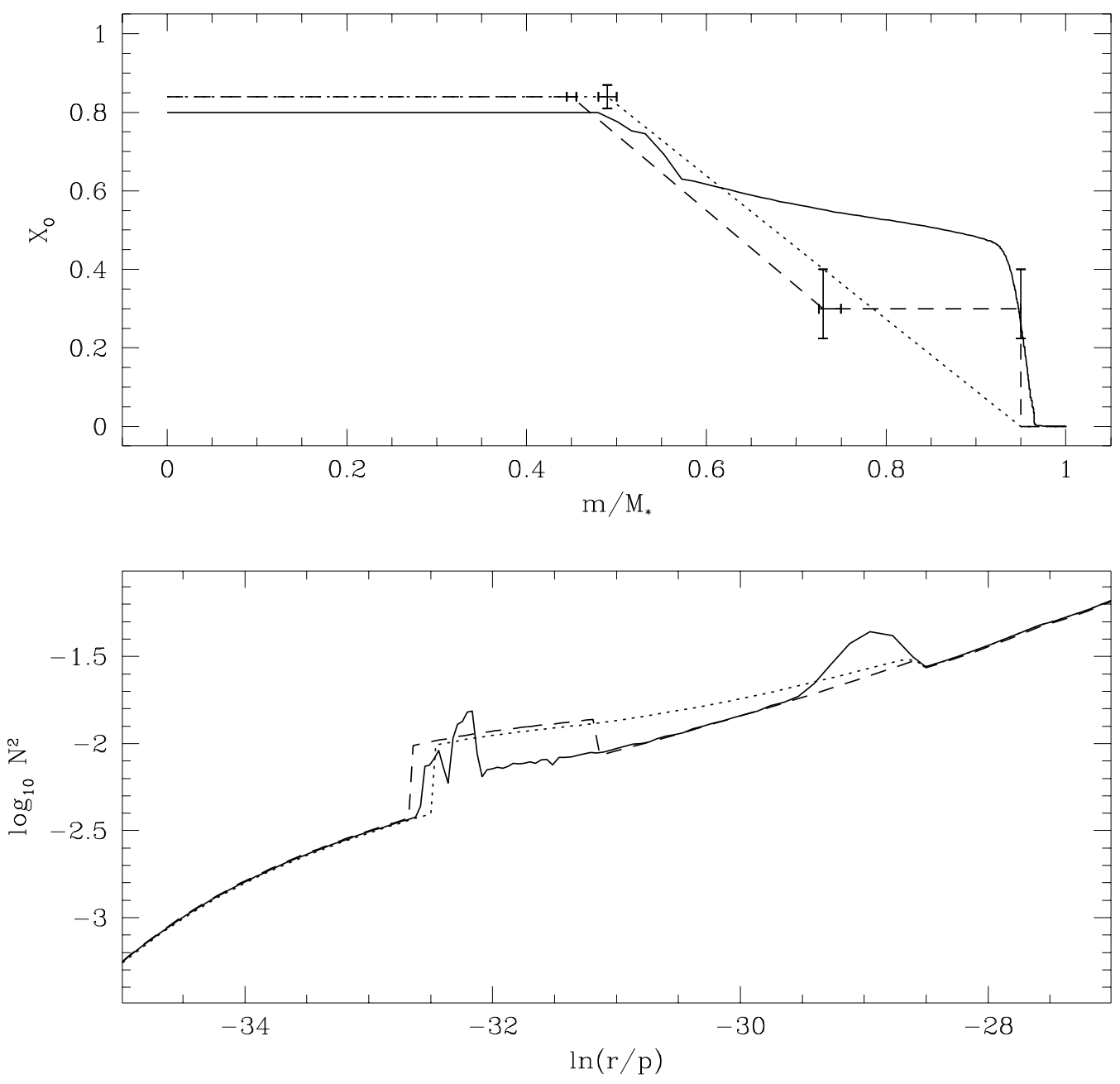

Figure 5.4: The internal oxygen profiles (top) and the corresponding region of the Brunt-Väisälä curves (bottom) for the best-fit forward model from $§ 4.6$ (dotted), the result of the best-fit reverse approach from $\S 5.2$ (dashed), and the scaled theoretical calculations of Salaris et al. (1997) for comparison (solid). 


\section{Chapter 6}

\section{Conclusions}

"The reasonable man adapts himself to the world; the unreasonable one persists in trying to adapt the world to himself. Therefore all progress depends on the unreasonable man."

- George Bernard Shaw

\subsection{Discussion of Results}

The application of genetic-algorithm-based optimization to white dwarf pulsation models has turned out to be very fruitful. We are now confident that we can rely on this approach to perform global searches and to provide not only objective, global best-fit models for the observed pulsation frequencies of DBV white dwarfs, but also fairly detailed maps of the parameter-space as a natural byproduct. This approach can easily be extended to treat the DAV stars and, with a grid of more detailed starter models, eventually the DOVs. Ongoing all-sky surveys promise to yield many new pulsating white dwarfs of all classes which will require follow-up with the Whole Earth Telescope to obtain seismological data. With the observation and analysis procedures in place, we will quickly be able to understand the statistical properties of these ubiquitous and relatively simple stellar objects. Our initial 3-parameter application of the method provided new evidence that the pulsation frequencies of white dwarfs really are global oscillations. We refined our knowledge of the sensitivity of the models to the structure 
of the envelope, and we demonstrated that they are sensitive to the conditions deep in the interior of the star, as suggested by previous work on crystallization by Montgomery \& Winget (1999).

The extension of the genetic-algorithm-based approach to optimize the internal composition and structure of our models of GD 358 yielded more exciting results. The values of the 3 parameters considered in the initial study $\left(M_{*}, T_{\text {eff }}, M_{\mathrm{He}}\right)$ were unchanged in the full 5-parameter fit, so we feel confident that they are the most important for matching the gross period structure. The efficiency of the GA relative to a grid search was much higher for this larger parameter-space, and the ability of the method to find the global best-fit was undiminished. The significant improvement to the fit made possible by including $X_{\mathrm{O}}$ and $q$ as free parameters confirms that the observed pulsations really do contain information about the hidden interiors of these stars.

Our best-fit solution has a thick helium layer, which should help to resolve the controversy surrounding the evolutionary connection between the PG 1159 stars and the DBVs. The helium layer mass for PG 1159-035 from the asteroseismological investigation of Winget et al. (1991) was relatively thick, at $\sim 3 \times 10^{-3} M_{\odot}$. Kleinman et al. (1998) found good agreement with the observed pulsations in the DAV star G29-38 using a similar helium layer mass. If the standard picture of white dwarf evolution is correct, with a slow cooling process connecting all three classes of pulsators, then we would expect a similar structure for the DBVs. The original best-fit model for GD 358 by Bradley \& Winget (1994b) had a relatively thin helium layer, at $\sim 1.2 \times 10^{-6} M_{\odot}$. This posed a problem for the standard picture. Dehner \& Kawaler (1995) treated this problem by including time-dependent diffusive processes in their calculations, but admitted that it still could not explain the presence of the DB gap, which is still an unresolved problem. Our thick envelope solution also fits more comfortably within the evolutionary scenario of a hot DB star becoming a carbon (DQ) white dwarf without developing an anomalously high photospheric carbon abundance (Provencal et al., 2000).

We have finally measured the central oxygen abundance in GD 358 and used it to provide a preliminary constraint on the ${ }^{12} \mathrm{C}(\alpha, \gamma){ }^{16} \mathrm{O}$ nuclear reaction crosssection. This reaction is one of the most important for understanding the late 
stages of stellar evolution and supernovae. Our preliminary value for the astrophysical S-factor at $300 \mathrm{keV}\left(S_{300}=290 \pm 15 \mathrm{keV}\right.$ barns $)$ is high relative to most published values. However, recent work on type Ia supernovae also favors a high value to produce model light curves with a sufficiently slow rise to maximum light (Hoeflich, Wheeler, \& Thielemann, 1998). Fortunately, there are other observational consequences of the higher rate in the spectra of type Ia supernovae models, so independent evidence should soon be possible.

More precise constraints on ${ }^{12} \mathrm{C}(\alpha, \gamma){ }^{16} \mathrm{O}$ from asteroseismology will require additional detailed simulations like those of Salaris et al. (1997). By determining the range of values for the cross-section that produce a central oxygen abundance within the measurement uncertainties of $X_{\mathrm{O}}$, we should be able to surpass the precision of the extrapolation from laboratory measurements by nearly an order of magnitude. The quoted uncertainty on our preliminary measurement of the ${ }^{12} \mathrm{C}(\alpha, \gamma){ }^{16} \mathrm{O}$ cross-section does not include systematic effects. There will certainly be some error associated with using our white dwarf models; we already know that they aren't perfect. There will also be some contribution to the uncertainty from the assumptions built in to the chemical profiles of Salaris et al. (1997), particularly from the description of convection.

We have demonstrated that the pulsation periods in our white dwarf models are sensitive to the shape of the internal chemical profiles. We can use this shape as a powerful diagnostic of other physical processes relevant to white dwarf model interiors, such as convective overshooting and crystallization.

While they are still embedded in the cores of red giant models, the internal chemical profiles of white dwarf models show a relatively constant $\mathrm{C} / \mathrm{O}$ ratio near the center, with a size determined by the extent of the helium-burning convective region. The degree of mixing at the edge of this region is unknown, so a convective overshooting parameter is used to investigate the effect of different assumptions about mixing. With no convective overshooting, the final $\mathrm{C} / \mathrm{O}$ ratio is constant out to the $50 \%$ mass point; with the convective overshooting parameter fixed at an empirically derived value, the central oxygen mass fraction is unchanged a the level of a few percent, but the region with a constant $\mathrm{C} / \mathrm{O}$ ratio extends out to the $65 \%$ mass point. Further out in both cases the oxygen mass fraction decreases as a result of the helium-burning shell moving toward the surface of 
the red giant model while gravitational contraction causes the temperature and density to rise. This increases the efficiency of the triple- $\alpha$ reaction, producing more carbon relative to oxygen.

Our parameterization of the internal chemical profile is not yet detailed enough to probe all of the physical information contained in the actual profiles. Our results suggest that convective overshooting is not required to explain the internal chemical profile of GD 358, to the extent that we can measure it at this time. Additional fitting with more detailed evolutionary profiles will provide a definite statement about convective overshooting, and will also provide constraints on the ${ }^{12} \mathrm{C}(\alpha, \gamma){ }^{16} \mathrm{O}$ reaction over the range of temperatures and densities sampled during the helium shell-burning phase.

Measurements of the internal chemical profiles will also provide a test of phase separation and crystallization in more massive or cooler pulsating white dwarf stars. The distribution of oxygen in the interior of a crystallizing white dwarf model is significantly different from the chemical profile during the liquid phase. The central oxygen mass fraction is higher, and the structure in the profile virtually disappears (Salaris et al., 1997).

Our constraints presently come from measurements of a single white dwarf star. Application of the GA fitting method to additional pulsating white dwarfs will provide independent determinations of the central $\mathrm{C} / \mathrm{O}$ ratio and internal chemical profiles. These measurements should lead to the same nuclear physics, or something is seriously wrong. Either way, we will learn something useful. It would be best to apply this technique to another DBV star before applying it to another class of pulsators, since it is still not certain that all of them are produced in the same way. If we were to find a significantly different $\mathrm{C} / \mathrm{O}$ ratio for another kind of pulsator, it could be telling us something about differences in the formation mechanisms.

The reverse approach to model-fitting has opened the door to exploring more complicated chemical profiles, and the initial results show qualitative agreement with recent theoretical calculations. We were originally motivated to develop this approach because the variance of our best-fit model from the forward method was still far larger than the observational uncertainty. This initial application has demonstrated the clear potential of the approach to yield better fits to the 
data, but the improvement to the residuals was only marginally significant. We should continue to develop this technique, but we must simultaneously work to improve the input physics of our models. In particular, we should really include oxygen in our envelopes and eventually calculate fully self-consistent models out to the surface.

\subsection{The Future}

\subsubsection{Next Generation Metacomputers}

In the short time since we finished building the specialized parallel computer that made the genetic algorithm approach feasible, processor and bus speeds have both quadrupled. At the same time, multiple-processor main boards have become significantly less expensive and operating systems have enhanced their shared memory multi-processing capabilities. These developments imply that a new metacomputer with only 16 processors on as few as 4 boards could now yield an equivalent computing power in a smaller space at a reduced price. There's no shame in this, it's just the nature of computer technology.

A famous empirical relation known as Moore's Law notes that computing speed doubles every 18 months. This has been true since the 1960's. A group at Steward Observatory recently used this relation to determine the largest calculation that should be attempted at any given time (Gottbrath et al., 1999). Their premise was that since computing power is always growing, it is sometimes more efficient to wait for technology to improve before beginning a calculation, rather than using the current technology. They found that any computation requiring longer than 26 months should not be attempted using presently available technology. We are happy to report that our calculations fell below this threshold, so we are better off today than we would have been if we had spent a year at the beach before building the metacomputer to do this project.

The guiding principle we used three years ago attempted to maximize the computing power of the machine per dollar. We now believe there are additional factors that should be considered. First, the marginal cost of buying presently available technology that will allow for easy upgrades in the future (especially 
faster processors) is small relative to the replacement cost of outdated parts. For a few hundred dollars extra, we could have bought $100 \mathrm{MHz}$ motherboards that would have allowed us to nearly triple the speed of the machine today for only the cost of the processors.

Second, the marginal cost of buying quality hardware (especially power supplies and memory) rather than the absolute cheapest hardware available is small relative to the cost of time spent replacing the cheap hardware. We actually learned this lesson before buying the hardware for the top row of 32 nodes. We have never had to replace a power supply in one of the top 32, but the bottom 32 still have power supply failures at the rate of one every two months or so.

Finally, because some hardware problems are inevitable, it pays to keep the number of nodes as small as possible. The marginal cost of slightly faster processors may be small compared to the time spent fixing problems on a larger number of nodes. True, when one of many nodes runs into a problem it has a smaller effect on the total computing power available, but the frequency of such problems is also higher. If we were to build another metacomputer today, we would estimate not only our budget in dollars, but also our budget in time.

\subsubsection{Code Adaptations}

Initially, we did not believe it would be possible to run the parallel genetic algorithm on supercomputers because, in its current form, the code dynamically spawns new tasks throughout the run. On local supercomputers at the University of Texas, software restrictions in the Cray implementation of PVM allow only a fixed number of slave tasks to be spawned, and only at the beginning of a run. This feature is intended to prevent any single job from dominating the resources.

Since the metacomputer provided a more cost effective solution to our computing requirements at the time, we never revisited the problem. We now believe that relatively minor modifications to the code would allow the slave jobs to remain active on a fixed number of processing elements and retain their essential function. This could allow us to solve much larger problems in a shorter time if we have access to supercomputers in the future.

Eventually, we hope to develop a more advanced version of the PIKAIA ge- 
netic algorithm. In particular, we'd like to incorporate a hybrid routine that would use a local hill-climbing scheme to speed up the convergence after the initial success of the genetic operators.

\subsubsection{More Forward Modeling}

In addition to the immediate application of forward modeling to more DBV white dwarfs, there are several possible extensions of the method to other types of white dwarf stars.

To use white dwarfs effectively as independent chronometers for stellar populations, we need to calibrate evolution models with observations of the internal structure of the coolest white dwarfs. The hydrogen-atmosphere variable (DAV) white dwarfs are the coolest class of known pulsators, so they can provide the most stringent constraints on the models.

Previous attempts to understand these objects have been hampered by their relatively sparse pulsation spectra. Kleinman et al. (1998) made repeated observations of the star G29-38 over many years and found a stable underlying frequency structure, even though only a subset of the full spectrum of modes were visible in each data set. Preliminary attempts to match the complete set of frequencies have focused on calculating grids of DAV models, but the huge range of possible parameters makes this task very computationally intensive. We hope to use the genetic-algorithm-based approach to explore the problem more efficiently.

Montgomery et al. (1999) showed that phase separation in crystallizing white dwarfs could add as much as $1.5 \mathrm{Gyr}$ to age estimates. With the discovery of the massive pulsator BPM 37093 by Kanaan et al. (1992), we now have the opportunity to test the theory of crystallization directly and calibrate this major uncertainty.

Like most other hydrogen-atmosphere white dwarfs, BPM 37093 exhibits a limited number of excited pulsation modes, but Nitta et al. (2000) secured reliable identifications of the spherical degree of these modes using the Hubble Space Telescope during a ground-based WET campaign. Preliminary attempts by Kanaan et al. (2000) to match the frequency structure revealed a parameter-correlation 
between the crystallized mass fraction and the thickness of the hydrogen layer.

The genetic algorithm is well equipped to deal with parameter-correlations. The initial application to GD 358 revealed correlations between several parameters and helped us to understand them in terms of the basic physical properties of the model. Despite the correlations, the genetic algorithm consistently found the global solution in every test using synthetic data, so we are confident that we will be able to use this method to separate unambiguously the effects of stellar crystallization from other model parameters.

\subsubsection{Ultimate Limits of Asteroseismology}

In our initial application of the reverse approach, we concentrated on only one region of the Brunt-Väisälä curve and we parameterized the perturbation to explore different possible internal chemical profiles efficiently. The technique is clearly useful, and we hope to use it to investigate a broad range of characteristics in our models that would be impractical to approach through forward modeling. In particular, we hope to quantify the ultimate limits of asteroseismology - to determine what we can learn from the data, and what we can never learn.

By using perturbations with various parameterizations, we may be able to probe weaknesses in the models themselves. We can address the question of what limitations we are imposing on our understanding simply by using the models we use. We may find that a whole range of models are pulsationally indistinguishable, or perhaps that our knowledge of certain regions of the model interior are limited only by the particular pulsation modes that we observe in the stars. It will be an entirely new way of looking at the problem, and it will give us the opportunity to learn even more from our data.

\subsection{Overview}

At the beginning of this project, we set out to learn something about nuclear fusion using pulsating white dwarf stars as a laboratory. What we've learned is unlikely to allow humanity to manufacture clean sustainable energy anytime soon; but the project has demonstrated that white dwarf asteroseismology has a 
clear potential to improve our knowledge of some fundamental nuclear physics. This is definitely a step in the right direction.

Along the way, we've developed some tools that hold great promise for the future of white dwarf asteroseismology and other computationally intensive modeling applications. We have developed a minimal-hardware design for a scalable parallel computer based on inexpensive off-the-shelf components. We have documented the server-side software configuration required to operate such a machine, and we have developed a generalized parallel genetic algorithm which can exploit the full potential of the hardware. We have modified well-established white dwarf evolution and pulsation codes to interface with the parallel GA and to provide stable operation over a broad range of interesting physical parameters.

We have laid the groundwork for new analyses that promise to unlock the secrets of the white dwarf stars. The fun has only started. 
Appendix A

Observations for the WET 


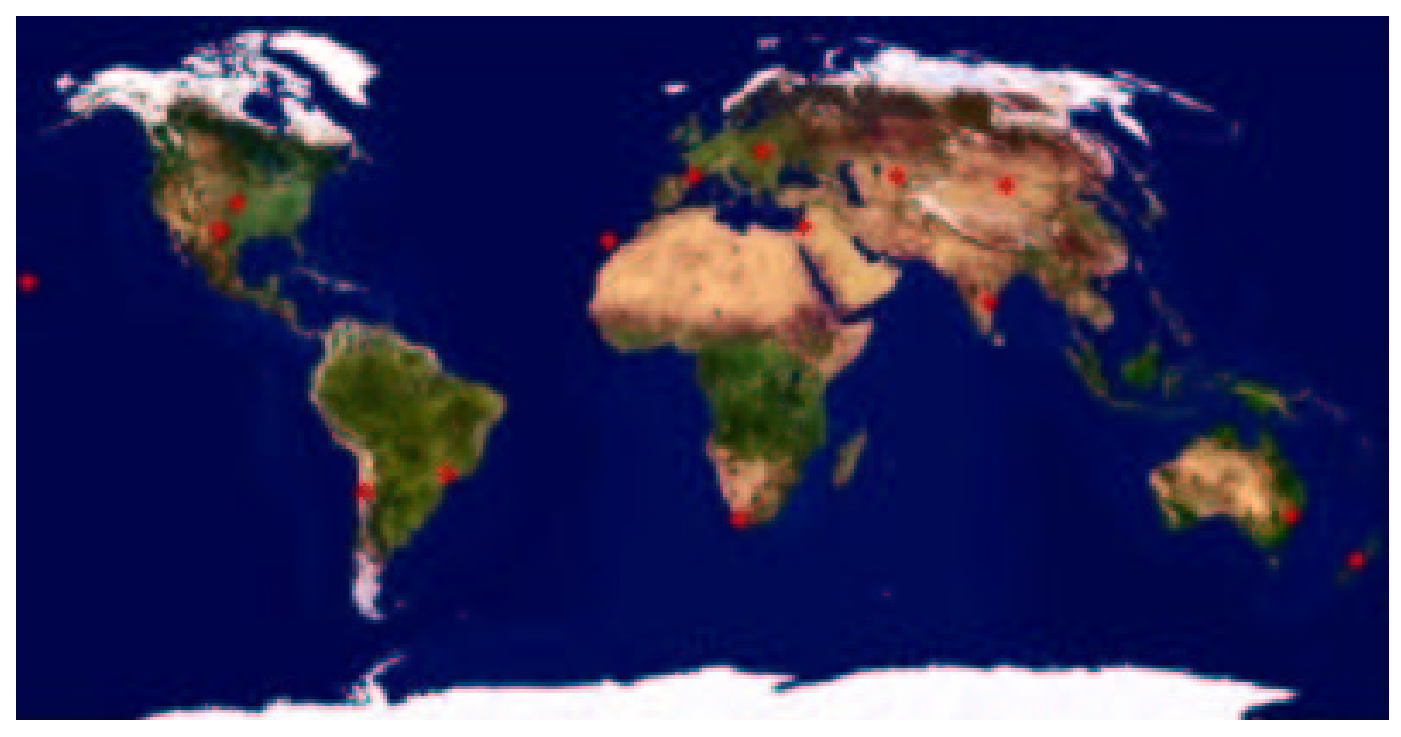

Figure A.1: The Whole Earth Telescope.

\section{A.1 What is the WET?}

The Whole Earth Telescope (WET) is an informal collaboration of astronomers at observatories around the world who cooperate to produce nearly continuous time-series photometry of white dwarfs and similar targets for up to 14 days at a time (see Figure A.1). This instrument has been operating since 1988, and is currently run from headquarters at Iowa State University.

During my time as a graduate student, I have participated in four organized campaigns to observe white dwarfs with the WET. Each campaign is referred to as XCOV (for extended coverage) followed by a number. XCOV 1 took place in March 1988. In every case but one, I was stationed at the 2.1-meter telescope at McDonald Observatory in west Texas. For XCOV 17, I used the 1.5-meter telescope at Cerro Tololo Interamerican Observatory. For the electronic edition of my dissertation, I have archived all of the raw observations that I obtained for each of these campaigns. 


\section{A.2 XCOV 15: DQ Herculis}

archive in digital dissertation:[tsm-0023 $\rightarrow$ tsm-0032]

\section{A.3 XCOV 17: BPM 37093}

archive in digital dissertation:[tsm-0033 $\rightarrow$ tsm-0048]

\section{A.4 XCOV 18: HL Tau 76}

archive in digital dissertation:[tsm-0049 $\rightarrow$ tsm-0072]

\section{A.5 XCOV 19: GD 358}

archive in digital dissertation:[tsm-0074 $\rightarrow$ tsm-0085] 
Appendix B

\section{Interactive Simulations}




\section{B.1 Pulsation Visualizations}

In digital dissertation: Spherical harmonic visualizations.

\section{B.2 The Effect of Viewing Angle}

In digital dissertation: Interactive demonstration of the effect of viewing angle on the amplitude of the observed brightness variations. 
Appendix C

Computer Codes 
This appendix contains an archive of the source code for the software that I have used for the calculations presented in this dissertation.

EVOLVE.F is a streamlined version of the White Dwarf Evolution Code (WDEC) described in $\S 4.3 .2$ with references to its origins and to the sources of data for the input physics. WDEC takes as input a hot starter model with a specific mass, which can come from detailed evolutionary calculations in the case of DOV stars, or from a simple polytropic approximation in the case of DBV and DAV stars. Using this starter model and other parameters specified in the header, WDEC adds an envelope with the specified composition and fractional mass and evolves the model quasi-statically until it reaches the specified temperature.

PULSATE.F uses the final output model produced by WDEC and calculates the $m=0$ adiabatic non-radial oscillation periods of a specified spherical degree $(\ell)$ within a specified period range. The periods resulting from the adiabatic approximation typically differ from the non-adiabatic results by only a few thousandths of a second, which is well below the present level of observational noise.

PVM_FITNESS.F is the code that uses the message-passing routines of the Parallel Virtual Machine (PVM) software to allow the public-domain genetic algorithm PIKAIA to evaluate the fitnesses of trials in parallel rather than sequentially. This code automatically determines the number of processors available for the calculation, balances the load when machines with differing speeds are used, and works around crashed jobs in a sensible way.

FF_SLAVE.F is an interface between the parallel genetic algorithm and the streamlined version of WDEC. This code runs on each machine that is used to calculate white dwarf models. It uses the message-passing routines of PVM to receive sets of parameters from the master process, evaluates the white dwarf model specified by those parameters, compares the model periods to the observations, and returns a measure of fitness to PIKAIA.

The practical aspects of running the evolution and pulsation codes are addressed in the documentation archive at the end of this appendix. 


\section{C.1 EVOLVE.F}

In digital dissertation: Hypertext version of evolution code.

\section{C.2 PULSATE.F}

In digital dissertation: Hypertext version of pulsation code. 


\section{C.3 PVM_FITNESS.F}

subroutine pvm_fitness (slave, num_jobs, npar, oldph, fitness)

c

c parallel fitness evaluation using PVM

c

implicit none

C

include '../include/fpvm3.h'

C

integer job, info, nhost, msgtype, iwhich, $i$

integer mytid, dtid, tids(0:128), flag, ntask

integer ttids(64), ptids(64), htids(64), flags(64)

integer speed, narch, numt, npar, nspawn, last, wait

integer num_jobs, ndone, length, par, trial, listen

integer finished(1024), resubmitted(1024)

C

double precision result, data(64)

real fitness (1024), oldph $(64,1024)$

C

character $* 40$ hostname

character*18 host

character*8 slave, arch

character*8 aout(64)

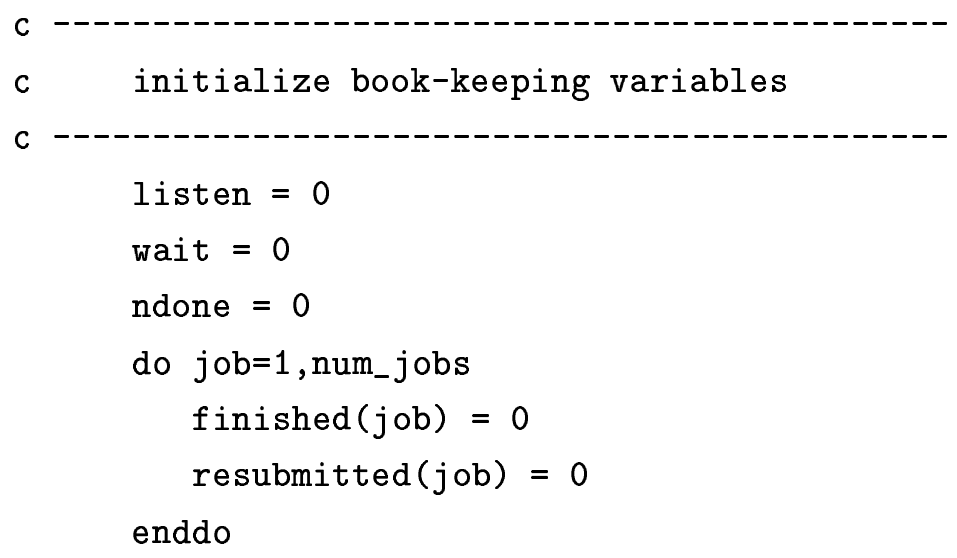




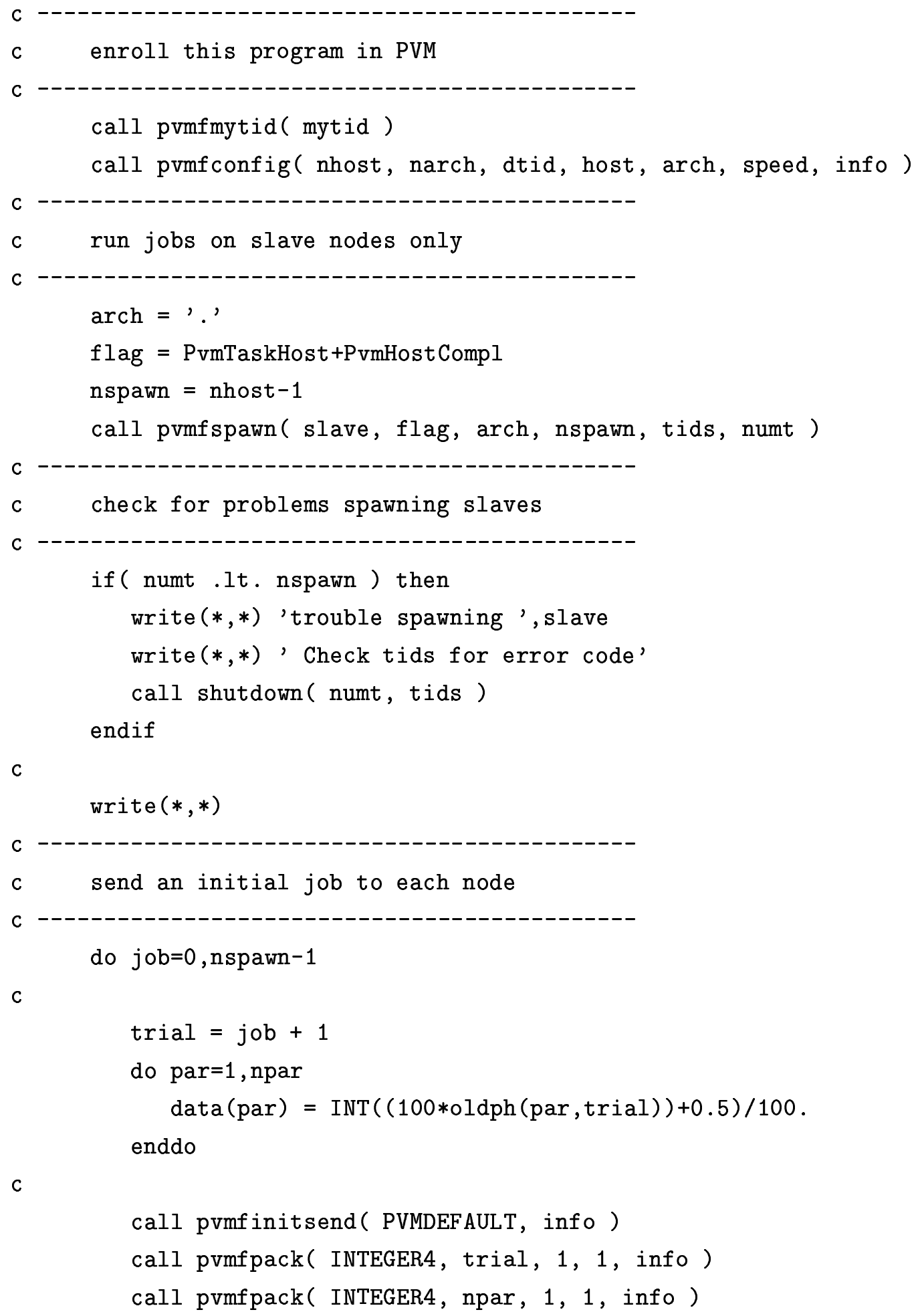

C

C

call pvmfinitsend( PVMDEFAULT, info )

call pvmfpack ( INTEGER4, trial, 1, 1, info )

call pvmfpack ( INTEGER4, npar, 1, 1, info ) 


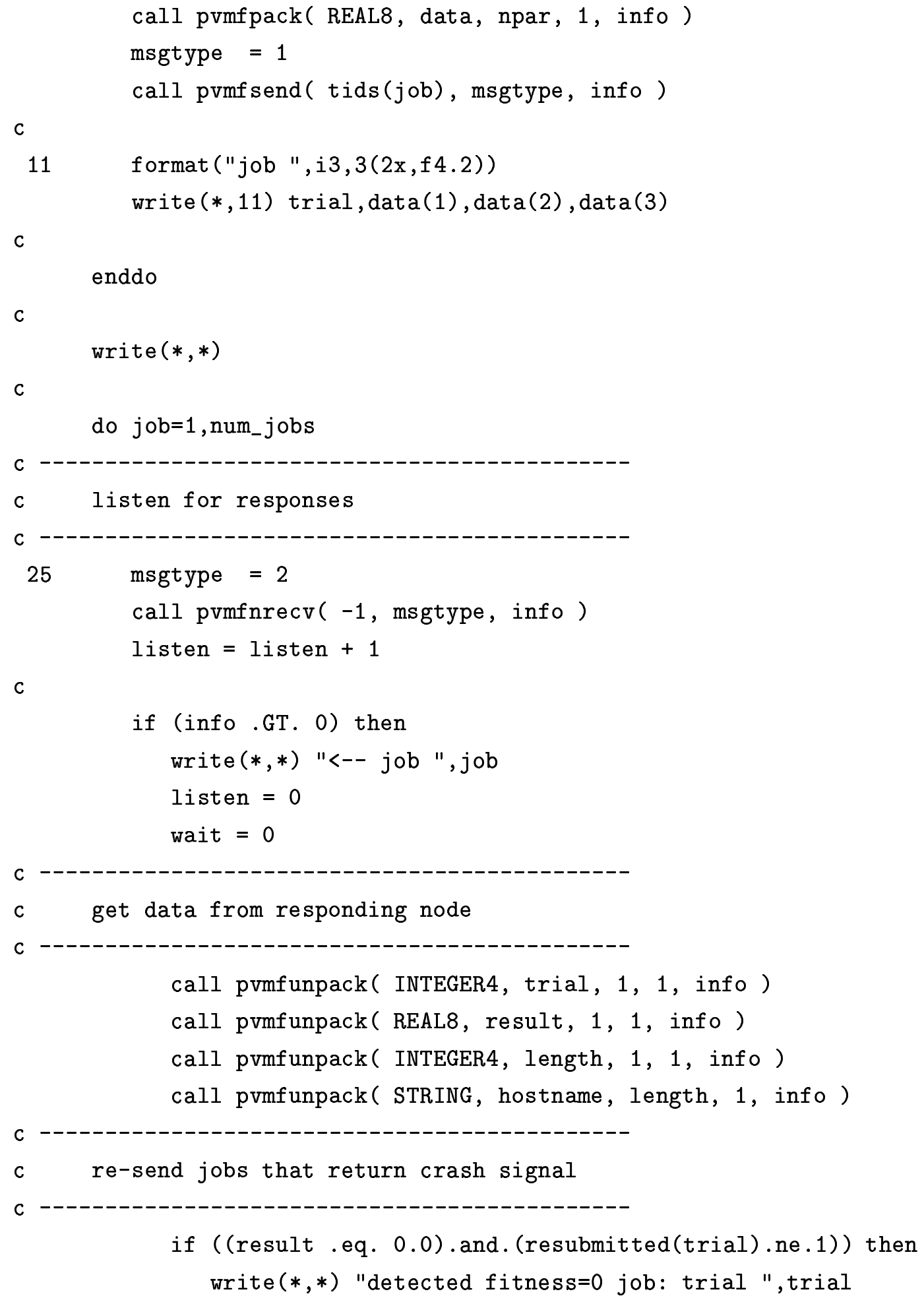




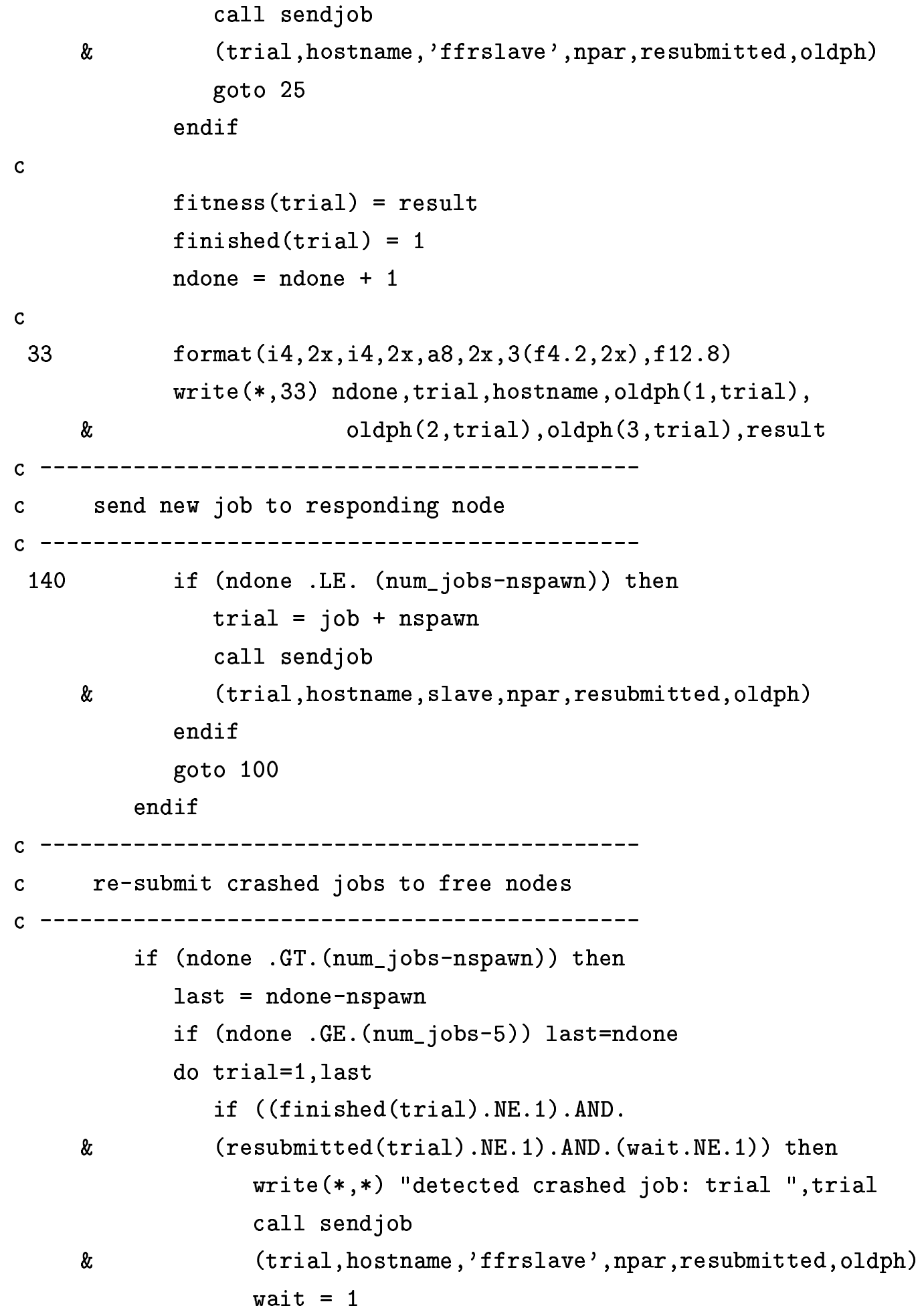




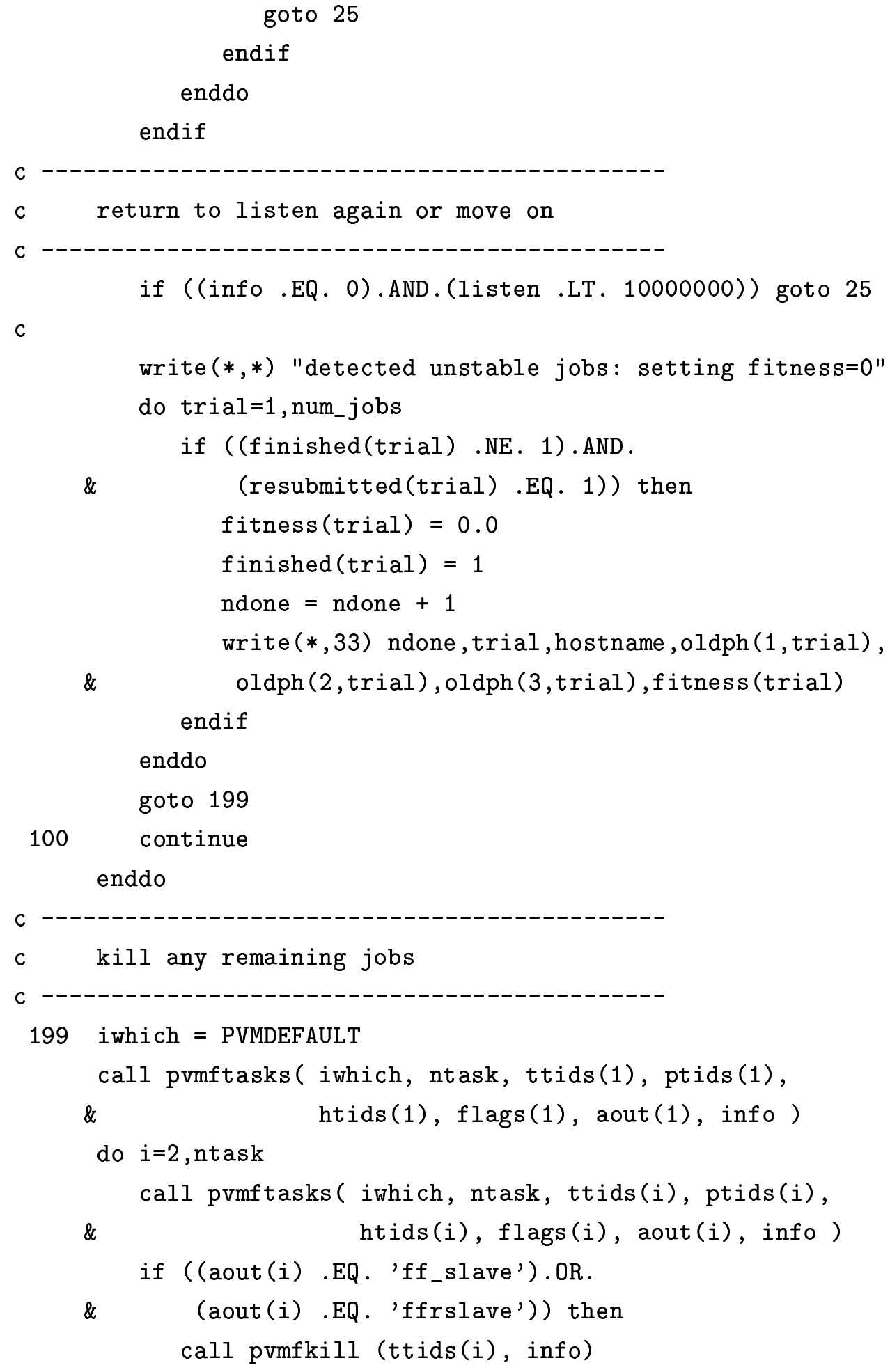


endif

enddo

c

call pvmfexit(info)

C

return

end

c $* * * * * * * * * * * * * * * * * * * * * * * * * * * * * * * * * * * * * * * * * * * * * * * * * * * * * * * * * * * * * * * * * * * * * * *$ subroutine sendjob(trial, hostname, slave,npar, resubmitted,oldph)

implicit none

include' .../include/fpvm3.h'

integer tids(0:128), numt, msgtype, par, npar, trial, info, flag integer resubmitted(1024)

double precision data(64)

real oldph $(64,1024)$

character*40 hostname

character*8 slave

C

call pvmfspawn( slave, 1, hostname, 1, tids, numt )

C

if ( numt .lt. 1) then

write $(*, *)$ 'trouble spawning', slave

write $(*, *)$ ' Check tids for error code'

call shutdown ( numt, tids )

endif

c

do $\operatorname{par}=1$, npar

$\operatorname{data}(\operatorname{par})=\operatorname{INT}((100 * 01 d p h(\operatorname{par}, \operatorname{trial}))+0.5) / 100$.

enddo

C 


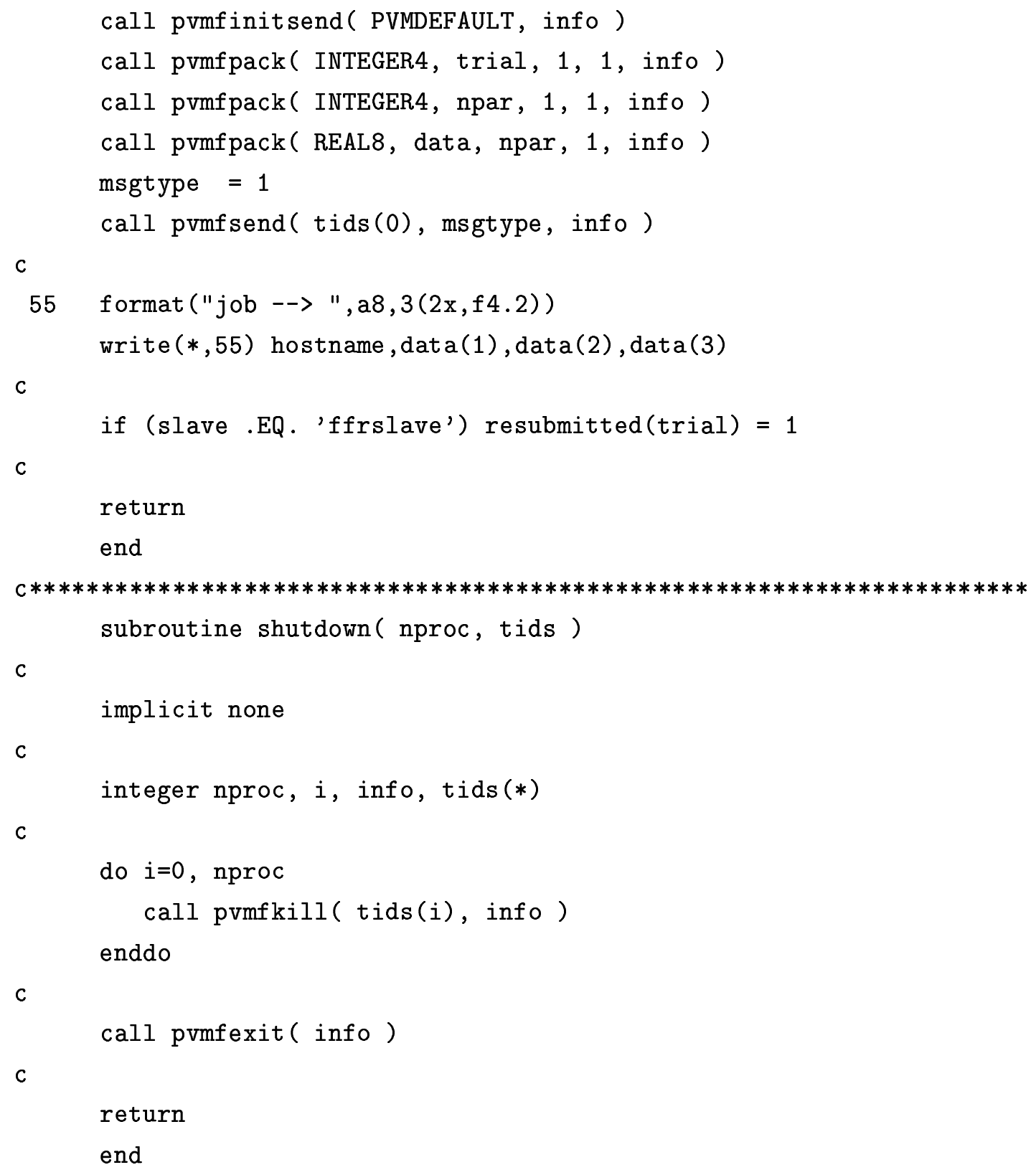




\section{C.4 FF_SLAVE.F}

program ff_slave

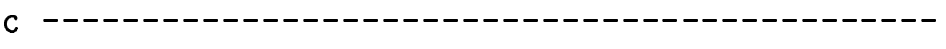

c fitness function slave program

c -------------------------------

implicit none

C

include '../include/fpvm3.h'

C

integer info, mytid, mtid, msgtype, speed, length, i

integer $\mathrm{n}$, nhost, narch, dtid, hostid, trial

C

double precision ff, data(32), result

C

character $* 40$ hostname, machine, arch

c -------------------------------------------

c enroll this program in PVM

call pvmfmytid( mytid)

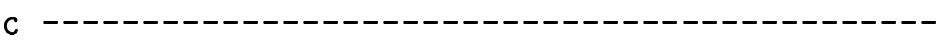

c get the master's task id

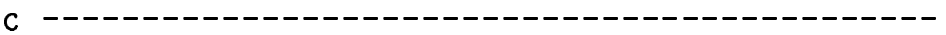

call pvmfparent ( mtid )

C

c receive data from master host

c

msgtype $=1$

call pvmfrecv( mtid, msgtype, info)

call pvmfunpack ( INTEGER4, trial, 1, 1, info )

call pvmfunpack ( INTEGER4, $\mathrm{n}, 1,1$, info )

call pvmfunpack ( REAL8, data, $\mathrm{n}, 1$, info )

C

c perform calculations with data

$\mathrm{c}$ 


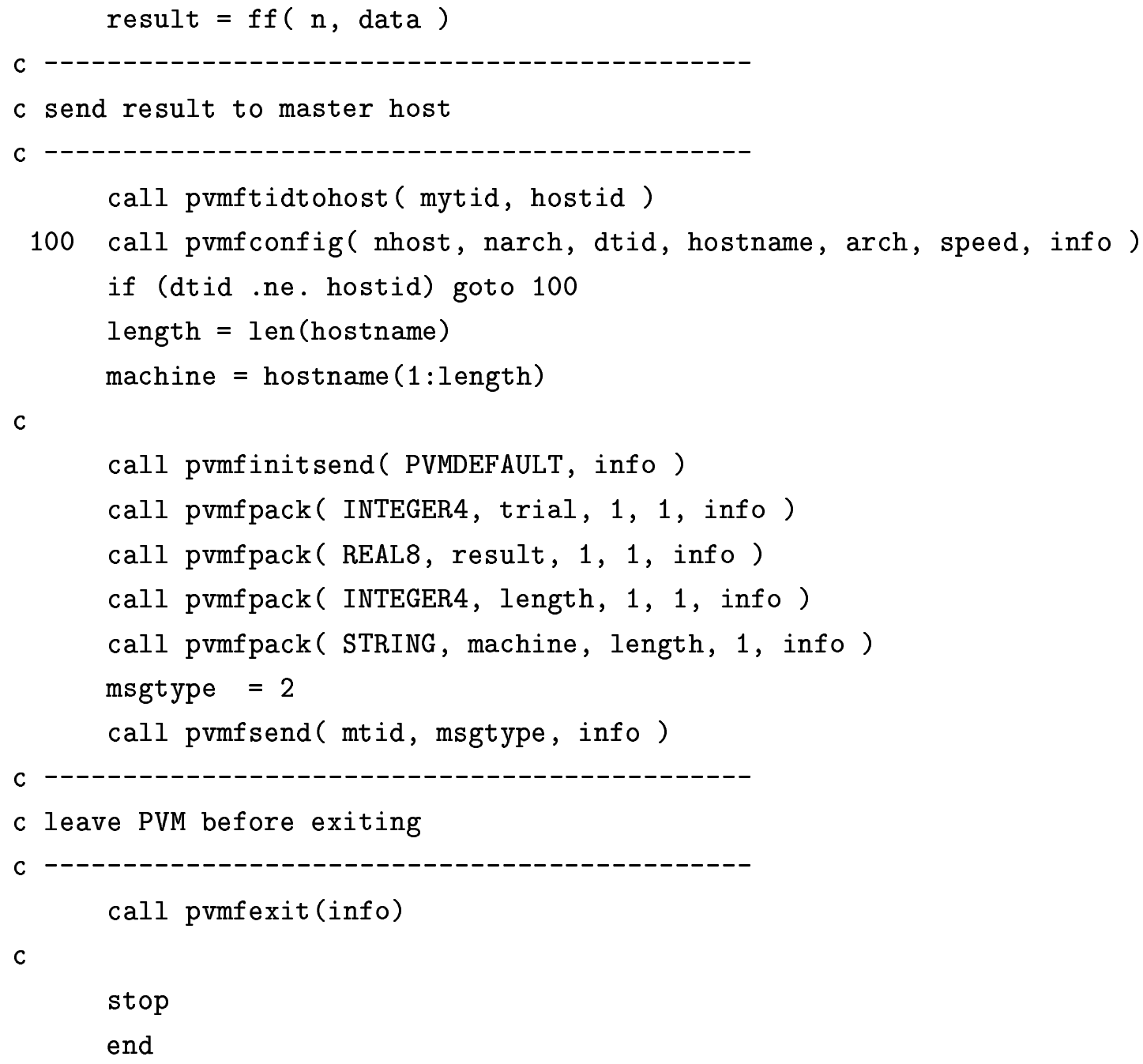




\section{C.5 Documentation}

In digital dissertation: Documentation archive.

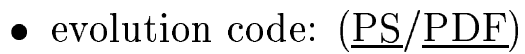

- prep code: ( $\underline{\mathrm{PS}} / \underline{\mathrm{PDF}})$

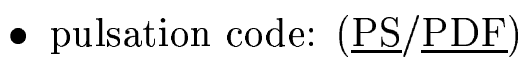




\section{Bibliography}

Arcoragi, J. \& Fontaine, G. 1980, ApJ, 242, 1208

Beauchamp, A., Wesemael, F., Bergeron, P., Fontaine, G., Saffer, R. A., Liebert, J. \& Brassard, P. 1999, ApJ, 516, 887

Becker, D., Sterling, T., Savarese, D., Dorband, J., Ranawak, U., \& Packer, C. 1995, in Proceedings of the International Conference on Parallel Processing (New York: Institute of Electrical and Electronics Engineers).

Bern, K. \& Wramdemark, S. 1973, Lowell Obs. Bull., No. 161

Böhm, K. H. \& Cassinelli, J. 1971, A\&A, 12, 21

Bradley, P. A. 1993, Ph.D. thesis, University of Texas-Austin

Bradley, P. A. \& Winget, D. E. 1994a, ApJ, 421, 236

Bradley, P. A. \& Winget, D. E. 1994b, ApJ, 430, 850

Bradley, P. A., Winget, D. E. \& Wood, M. A. 1993, ApJ, 406, 661

Brassard, P., Fontaine, G., Wesemael, F. \& Hansen, C. J. 1992, ApJS, 80, 369

Buchmann, L. 1996, ApJ, 468, L127

Caughlan, G. R., Fowler, W. A., Harris, M. J., \& Zimmerman, B. A. 1985, Atomic Data and Nuclear Data Tables, 32, 197

Charbonneau, P. 1995, ApJS, 101, 309

Charbonneau, P., Tomczyk, S., Schou, J., Thompson, M.J. 1998, ApJ, 496, 1015 
Crick, F. 1984, quoted by Daniel C. Dennett in Elbow Room: the varieties of free will worth wanting (Cambridge, MA: MIT Press)

Darwin, C. 1859, The Origin of Species (New York: Penguin Books)

Dehner, B. T. \& Kawaler, S. D. 1995, ApJ, 445, L141

Feynman, R. P., Leighton, R. B. \& Sands, M. 1963, The Feynman Lectures on Physics, Vol. 1 (Reading, MA: Addison-Wesley)

Fontaine, G., Graboske, H. C., Jr., \& Van Horn, H. M. 1977, ApJS, 35, 293

Fowler, W. A. 1986, in Highlights of Modern Physics, ed. S. L. Shapiro \& S. A. Teukolsky (New York: John Wiley \& Sons), 3

Geist, A., Beguelin, A., Dongarra, J., Jiang, W., Manchek, R., \& Sunderam, V. 1994, PVM: Parallel Virtual Machine, A Users' Guide and Tutorial for Networked Parallel Computing, (Cambridge: MIT Press)

Goldberg, D. 1989, Genetic Algorithms in Search, Optimization, and Machine Learning, (Reading, MA: Addison-Wesley).

Gottbrath, C. et al. 1999, http://arxiv.org/abs/astro-ph/9912202

Greenstein, J. L. 1969, ApJ, 158, 281

Hakala, P. 1995, A\&A, 296, 164

Harrington, R. S., Kallarakal, V. V., Christy, J. W., Dahn, C. C., Riepe, B. Y., Guetter, H. H., Ables, H. D., Hewitt, A. V., Vrba, F. J., \& Walker, R. L. 1985, AJ, 90, 123

Hoeflich, P., Wheeler, J. C., \& Thielemann, F. K. 1998, ApJ, 495, 617

Iglesias, C. A., \& Rogers, F. J. 1993, ApJ, 412, 752

Itoh, N., Hayashi, H., Nishikawa, A., \& Kohyama, Y. 1996, ApJS, 102, 411

Kallrath, J. \& Linnell, A. P. 1987, ApJ, 313, 346 
Kanaan, A., Kepler, S. O., Giovannini, O., \& Diaz, M. 1992, ApJ, 390, L89

Kanaan, A., Nitta-Kleinman, A., Winget, D. E., Kepler, S. O., Montgomery, M., \& WET team 2000, Baltic Astronomy, 9, 87

Kawaler, S. 1986, Ph.D. thesis, University of Texas-Austin

Kawaler, S. D. 1990, ASP Conf. Ser. 11: Confrontation Between Stellar Pulsation and Evolution, 494

Kawaler, S. \& Weiss, P. 1990, in Proc. Oji International Seminar, Progress of Seismology of the Sun and Stars, ed. Y. Osaki \& H. Shibahashi (Berlin: Springer), 431

Kennelly, E. J., Walker, G. A. H., Catala, C., Foing, B. H., Huang, L., Jiang, S., Hao, J., Zhai, D., Zhao, F., Neff, J. E., Houdebine, E. R., Ghosh, K. K., Charbonneau, P. 1996, A\&A, 313, 571.

Kleinman, S. J., Nather, R. E., Winget, D. E., Clemens, J. C., Bradley, P. A., Kanaan, A., Provencal, J. L., Claver, C. F., Watson, T. K., Yanagida, K., Nitta, A., Dixson, J. S., Wood, M. A., Grauer, A. D., Hine, B. P., Fontaine, G., Liebert, J., Sullivan, D. J., Wickramasinghe, D. T., Achilleos, N., Marar, T. M. K., Seetha, S., Ashoka, B. N., Meistas, E., Leibowitz, E. M., Moskalik, P., Krzesinski, J., Solheim, J. -., Bruvold, A., O’Donoghue, D., Kurtz, D. W., Warner, B., Martinez, P., Vauclair, G., Dolez, N., Chevreton, M., Barstow, M. A., Kepler, S. O., Giovannini, O., Augusteijn, T., Hansen, C. J., \& Kawaler, S. D. 1998, ApJ, 495, 424

Kutter, G. S. \& Savedoff, M. P. 1969, ApJ, 156, 1021

Lamb, D. Q. 1974, Ph.D. thesis, University of Rochester

Lamb, D. Q. \& Van Horn, H. M. 1975, ApJ, 200, 306

Lang, M. 1995, Ir. Astron. J., 22, 167

Lazio, T. 1997, PASP, 109, 1068

Metcalfe, T. S. 1999, AJ, 117, 2503 
Metcalfe, T. S., \& Nather, R. E. 1999, Linux Journal, 65, 58

Metcalfe, T. S., \& Nather, R. E. 2000, Baltic Astronomy, 9, 479

Metcalfe, T. S., Nather, R. E. \& Winget, D. E. 2000, ApJ, 545, 974

Montgomery, M. H. 1998, Ph.D. thesis, University of Texas-Austin

Montgomery, M. H., Klumpe, E. W., Winget, D. E., \& Wood, M. A. 1999, ApJ, 525,482

Montgomery, M. H., Metcalfe, T. S. \& Winget, D. E. 2001, ApJ, 548, L53

Montgomery, M. H. \& Winget, D. E. 1999, ApJ, 526, 976

Napiwotzki, R., Green, P. J. \& Saffer, R. A. 1999, ApJ, 517, 399

Nather, R. E., Winget, D. E., Clemens, J. C., Hansen, C. J. \& Hine, B. P. 1990, ApJ, 361, 309

Nitta, A., Kanaan, A., Kepler, S. O., Koester, D., Montgomery, M. H., \& Winget, D. E. 2000, Baltic Astronomy, 9, 97

Proctor, D. D. \& Linnell, A. P. 1972, ApJS, 24, 449

Proust, M. 1981, in Readers Digest, 24 February (Pleasantville, NY: The Reader's Digest Association, Inc.)

Provencal, J. L., Shipman, H. L., Thejll, P., \& Vennes, S. 2000, ApJ, 542, 1041

Salaris, M., Dominguez, I., Garcia-Berro, E., Hernanz, M., Isern, J. \& Mochkovitch, R. 1997, ApJ, 486, 413

Shaw, G. B. 1903, Man and Superman; a comedy and a philosophy (Westminster: A. Constable)

Sweigart, A., Greggio, L. \& Renzini, A. 1990, ApJ, 364, 527

Tassoul, M., Fontaine, G., \& Winget, D. E. 1990, ApJS, 72, 335 
Torvalds, L. 1999, interview on CNN \& Fortune, 24 February (http://digistar.com/luclist/1999-02/0032.html)

Vuille, F., O'Donoghue, D., Buckley, D. A. H., Massacand, C. -., Solheim, J. E., Bard, S., Vauclair, G., Giovannini, O., Kepler, S. O., Kanaan, A., Provencal, J. L., Wood, M. A., Clemens, J. C., Kleinman, S. J., O'Brien, M. S., Nather, R. E., Winget, D. E., Nitta, A., Klumpe, E. W., Montgomery, M. H., Watson, T. K., Bradley, P. A., Sullivan, D. J., Wu, K., Marar, T. M. K., Seetha, S., Ashoka, B. N., Mahra, H. S., Bhat, B. C., Babu, V. C., Leibowitz, E. M., Hemar, S., Ibbetson, P., Mashals, E., Meištas, E., Moskalik, P., Zoła, S., Krzesiński, J., \& Pajdosz, G. 2000, MNRAS, 314, 689

Wahde, M. 1998, A\&AS, 132, 417

Wampler, J. 1973, quoted by Ed Nather in 1995, Baltic Astronomy, 4, 124

Wegner, G. 1979, in IAU Colloquium 53, White Dwarfs and Variable Degenerate Stars (Rochester, New York: University of Rochester), 250

Winget, D. E. 1981, Ph.D. thesis, University of Rochester

Winget, D. E., Robinson, E. L., Nather, R. D. \& Fontaine, G. 1982, ApJ, 262, L11

Winget, D. E., Nather, R. E., Clemens, J. C., Provencal, J. L., Kleinman, S. J., Bradley, P. A., Claver, C. F., Dixson, J. S., Montgomery, M. H., Hansen, C. J., Hine, B. P., Birch, P., Candy, M., Marar, T. M. K., Seetha, S., Ashoka, B. N., Leibowitz, E. M., O'Donoghue, D., Warner, B., Buckley, D. A. H., Tripe, P., Vauclair, G., Dolez, N., Chevreton, M., Serre, T., Garrido, R., Kepler, S. O., Kanaan, A., Augusteijn, T., Wood, M. A., Bergeron, P., \& Grauer, A. D. 1994, ApJ, 430, 839

Winget, D. E., Nather, R. E., Clemens, J. C., Provencal, J., Kleinman, S. J., Bradley, P. A., Wood, M. A., Claver, C. F., Frueh, M. L., Grauer, A. D., Hine, B. P., Hansen, C. J., Fontaine, G., Achilleos, N., Wickramasinghe, D. T., Marar, T. M. K., Seetha, S., Ashoka, B. N., O'Donoghue, D., Warner, B., Kurtz, D. W., Buckley, D. A., Brickhill, J., Vauclair, G., Dolez, N., Chevreton, 
M., Barstow, M. A., Solheim, J. E., Kanaan, A., Kepler, S. O., Henry, G. W., \& Kawaler, S. D. 1991, ApJ, 378, 326

Wood, M. 1990, Ph.D. thesis, University of Texas-Austin

Woosley, S. E., Timmes, F. X. \& Weaver, T. A. 1993, in Nuclei in the Cosmos, Vol. 2, ed. F. Kappeler \& K. Wisshak (Philadelphia: IOP), 531 


\section{Vita}

Travis Scott Metcalfe was born in Seaside, Oregon on 12 October 1973. He lived on the central Oregon coast with his parents Jerry and Karen until graduating from Newport High School in 1991. In August of that year, he moved to Tucson, Arizona to study Astronomy and Physics at the University of Arizona, where he received a Bachelor of Science degree in May 1996. He came to Austin, Texas in August 1996 to begin graduate studies in the Department of Astronomy at the University of Texas where he received a Master of Arts degree two years later.

Permanent Address: 3013 Harris Park Avenue, Austin, Texas 78705

This dissertation was typeset by the author. 